\title{
Making sense of dislocation correlations
}

\author{
Thomas Hochrainer* (D), Benedikt Weger and Satyapriya Gupta
}

\author{
*Correspondence: \\ hochrainer@tugraz.at \\ Technische Universität Graz, Institut \\ für Festigkeitslehre, \\ Kopernikusgasse 24, $8010 \mathrm{Graz}$, \\ Austria
}

\begin{abstract}
Since crystal plasticity is the result of moving and interacting dislocations, it seems self-evident that continuum plasticity should in principle be derivable as a statistical continuum theory of dislocations, though in practice we are still far from doing so. One key to any statistical continuum theory of interacting particles is the consideration of spatial correlations. However, because dislocations are extended one-dimensional defects, the classical definition of correlations for point particles is not readily applicable to dislocation systems: the line-like nature of dislocations entails that a scalar pair correlation function does not suffice for characterizing spatial correlations and a hierarchy of two-point tensors is required in general. The extended nature of dislocations as closed curves leads to strong self-correlations along the dislocation line. In the current contribution, we thoroughly introduce the concept of pair correlations for general averaged dislocation systems and illustrate self-correlations as well as the content of low order correlation tensors using a simple model system. We furthermore detail how pair correlation information may be obtained from three-dimensional discrete dislocation simulations and provide a first analysis of correlations from such simulations. We briefly discuss how the pair correlation information may be employed to improve existing continuum dislocation theories and why we think it is important for analyzing discrete dislocation data.
\end{abstract}

Keywords: Crystal plasticity, Dislocation correlations, Continuum theory of dislocations, Discrete dislocation dynamics

\section{Introduction}

Plastic deformation of crystals on the microscale is mainly mediated by the motion and interactions of dislocations. However, the macroscopic plastic properties, most notably the flow stress, hardening characteristics, and the emergence of dislocation structures are seldom a direct consequence of the characteristics of individual dislocations, but arise from their collective behavior rooted in the elastic interactions between dislocations. In the context of a possible statistical continuum theory of crystal plasticity based on dislocations, this implies that a mean field approximation based on single-point densities will usually not be sufficient and two-point data, that is dislocation correlations, needs to be taken into account. This has been early recognized by E. Kröner (1969), who suggested a series of correlation tensors generalizing his earlier proposed dislocation density tensor (Kröner 1958), see also Kröner (2001). However, it was not before the advent of powerful computer simulations of discrete dislocations, that dislocation correlations could be

(c) The Author(s). 2022 Open Access This article is licensed under a Creative Commons Attribution 4.0 International License, which permits use, sharing, adaptation, distribution and reproduction in any medium or format, as long as you give appropriate credit to the original author(s) and the source, provide a link to the Creative Commons licence, and indicate if changes were made. The images or other third party material in this article are included in the article's Creative Commons licence, unless indicated otherwise in a credit line to the material. If material is not included in the article's Creative Commons licence and your intended use is not permitted by statutory regulation or exceeds the permitted use, you will need to obtain permission directly from the copyright holder. To view a copy of this licence, visit http://creativecommons.org/licenses/by/4.0/. 
determined at least in the special case of straight parallel edge dislocations, such that a first statistical continuum theory of straight dislocations could be developed in the early 2000s (Zaiser et al. 2001; Groma et al. 2003). This approach reached full maturity recently (Valdenaire et al. 2016; Groma et al. 2016; Wu et al. 2018), by considering correlation information which had been discarded earlier.

As for the development of a three-dimensional statistical continuum theory of curved dislocations, already characterizing the dislocation state with continuum variables and capturing the kinematics of moving curves in evolution equations for these variables posed a considerable challenge. Inspired by the success of the 2D theory and initial progress towards a 3D theory, there have been a few attempts in the late 2000s to obtain and evaluate pair correlations from three-dimensional Discrete Dislocation Dynamics (DDD) simulations (Deng and El-Azab 2007a; Csikor et al. 2007; Deng and El-Azab 2007b); however, it seems fair to state that all of them remained rather preliminary. The topic has been largely left aside since then, which could be attributed to several reasons: on the theoretical side, upon having fixed the kinematics, prospects in Continuum Dislocation Dynamics (CDD) shifted to a more phenomenological, energetic approach on the one hand (Groma et al. 2007; Hochrainer 2016; Hochrainer 2017; Groma et al. 2021), and the direct incorporation of various dislocation reactions into continuum theories on the other hand (Xia et al. 2016; Lin and El-Azab 2020; Sudmanns et al. 2019). On the more practical side, the early attempts demonstrated that much more extensive DDD data would be required for sufficient statistics and we dare stating that there was and still is a shortage of knowledge about how to evaluate DDD data and what to look for in terms of correlations. The issue of 3D dislocation correlations was taken up again recently by Anderson and El-Azab (2021). However, in this work the authors restrict themselves to a special ensemble leading to the so-called line density approximation, which entails a specific definition of correlations. This definition is fundamentally different from how correlations have been studied in systems of straight parallel edge dislocations and therefore this approach does not lend itself for transferring the latter methods to general systems of curved dislocations. To provide the basis for this generalization and to showcase the analysis of correlations from DDD simulations are the primary objectives of the current contribution.

The foundations for characterizing dislocation correlations in systems of curved dislocations have already been laid by Zaiser (2015), where he proposed a series of correlation tensors generalizing the concept of alignment tensors introduced for the single-point dislocation measures in Hochrainer (2015). Though Zaiser suggested obtaining these correlation tensors as the necessary next step, he did not describe how to do this in practice nor did he provide any details about the content of the tensors and their interpretation. Another topic which has not yet received the attention it deserves is that the fact that dislocations are connected lines entails strong self-correlations; though these have been addressed for model systems already in the 1980s by Stoyan and Stoyan (1986). That these self-correlations indeed dominate correlations obtained from DDD simulations has been shown in preliminary form by Csikor et al. (2007).

The current paper provides an accessible introduction into the peculiarities of dislocation correlations, where the line-like nature of dislocations entails both, the tensorial character of correlations and the strong self-correlations. In the exposition on the correlation tensors presented in Section Dislocation density and correlation tensors, emphasis 
is given on the consequences of evaluating correlations as functions of the distance vector between points on discrete dislocations. In Section Correlations in 'uncorrelated' distributions of circular loops, we discuss in detail the self-correlations due to connectivity in a two-dimensional model system of circular loops, which simultaneously serves the purpose of providing a notion of the content of some of the low-order correlation tensors. Subsequently, in Section Correlation tensor evaluation from discrete dislocation data, the practical determination of correlations from DDD codes is introduced. The algorithm is verified and showcased at an artificial ensemble corresponding to the analytical case discussed in the preceding section, before it is applied to relaxed dislocation configurations of a single slip system. With the help of these results, we eventually discuss how to identify and isolate the self-correlations inevitably contained in correlation data obtained from DDD simulations. We summarize and discuss the results in Section Summary, discussion, and outlook, where we also provide a brief outlook on how to employ findings on correlations in plasticity research.

\section{Dislocation density and correlation tensors}

In the following, we discuss the definition of spatial correlations using ensemble averages of spatially homogeneous systems of dislocations. We assume an ensemble of realizations which is generated by some sort of actual or virtual experiment. The realizations are dislocation structures in a region $\Omega$ that share suitable macroscopic characteristics which may include characteristics of the preparation process and/or dislocation density variables, for example, the total dislocation density. Examples of such ensembles are provided in Section Correlation tensor evaluation from discrete dislocation data.

We assume that one can decompose any dislocation configuration into a countable number of oriented curve segments $c_{m}^{\boldsymbol{b}}$, where $m \in\{1, \ldots, M(\boldsymbol{b})\}$ and $M(\boldsymbol{b})$ denotes the number of dislocation segments with Burgers vector $\boldsymbol{b}$. Note that $M(\boldsymbol{b})$ will be different for different realizations, but we do not indicate this in the current notation. Every realization induces a discrete density which we write as integral of Dirac-delta distributions along the curve segments, such that

$$
\boldsymbol{\rho}^{\mathrm{d}}(\boldsymbol{x})=\sum_{\boldsymbol{b}} \sum_{m=1}^{M(\boldsymbol{b})} \int_{c_{m}^{\boldsymbol{b}}} \delta\left(\boldsymbol{x}-\boldsymbol{c}_{m}^{\boldsymbol{b}}(s)\right) \partial_{s} \boldsymbol{c}_{m}^{\boldsymbol{b}}(s) \mathrm{d} s=\sum_{\boldsymbol{b}} \sum_{m=1}^{M(\boldsymbol{b})} \int_{c_{m}^{b}} \delta\left(\boldsymbol{x}-\boldsymbol{c}_{m}^{\boldsymbol{b}}(s)\right) \mathrm{d} \boldsymbol{c},
$$

where

$$
\mathrm{d} \boldsymbol{c}=\partial_{s} \boldsymbol{c}_{m}^{\boldsymbol{b}}(s) \mathrm{d} s
$$

is the vectorial line-element of the curve. Relation (1) therefore defines a vectorial distribution which we consider the natural distributional representation for oriented curves like dislocations. Before discussing additional distributional representations, we note that the integral of $\rho^{\mathrm{d}}$ over a volume $V \subset \Omega$ yields

$$
\int_{V} \boldsymbol{\rho}^{\mathrm{d}}(\boldsymbol{x}) \mathrm{d} \boldsymbol{x}=\sum_{\boldsymbol{b}} \sum_{m=1}^{M(\boldsymbol{b})} \int_{c_{m}^{\boldsymbol{b}} \cap V} \mathrm{~d} \boldsymbol{c}=\sum_{\boldsymbol{b}} \sum_{m=1}^{M(\boldsymbol{b})}\left(\boldsymbol{c}_{m}^{\boldsymbol{b}}\left(s_{\otimes}(V)\right)-\boldsymbol{c}_{m}^{\boldsymbol{b}}\left(s_{\odot}(V)\right)\right),
$$

i.e., the sum over the vectors connecting the points where the curve segment enters, $\boldsymbol{c}_{m}^{\boldsymbol{b}}\left(s_{\odot}(V)\right)$, and leaves, $\boldsymbol{c}_{m}^{\boldsymbol{b}}\left(s_{\otimes}(V)\right)$, the volume $V$. In case that the segment starts and/or ends inside the volume, $\boldsymbol{c}_{m}^{\boldsymbol{b}}\left(s_{\odot}(V)\right)$ and $\boldsymbol{c}_{m}^{\boldsymbol{b}}\left(s_{\otimes}(V)\right)$ may denote the start and the end-point in the volume, respectively. 
In the sequel we classify dislocations by their Burgers vector and introduce the vector densities

$$
\boldsymbol{\rho}^{\mathrm{d}, \boldsymbol{b}}(\boldsymbol{x})=\sum_{m=1}^{M(\boldsymbol{b})} \int_{c_{m}^{\boldsymbol{b}}} \delta\left(\boldsymbol{x}-\boldsymbol{c}_{m}^{\boldsymbol{b}}(s)\right) \mathrm{d} \boldsymbol{c} .
$$

We shall occasionally also refer to these distributional vector densities (and their continuum counterparts obtained from ensemble averaging) as geometrically necessary dislocation (GND) vectors. The notion is motivated from their constitutive role for the distributional dislocation density tensor, which is defined as (e.g. Kröner (2001))

$$
\boldsymbol{\alpha}^{\mathrm{d}}(\boldsymbol{x})=\sum_{\boldsymbol{b}} \boldsymbol{\rho}^{\mathrm{d}, \boldsymbol{b}}(\boldsymbol{x}) \otimes \boldsymbol{b} .
$$

Upon integrating this tensor over a volume we obtain the geometrically necessary dislocation content,

$$
\int_{V} \boldsymbol{\alpha}^{\mathrm{d}}(\boldsymbol{x}) \mathrm{d} \boldsymbol{x}=\sum_{\boldsymbol{b}} \sum_{m=1}^{M(\boldsymbol{b})}\left(\boldsymbol{c}_{m}^{\boldsymbol{b}}\left(s_{\otimes}(V)\right)-\boldsymbol{c}_{m}^{\boldsymbol{b}}\left(s_{\odot}(V)\right)\right) \otimes \boldsymbol{b} .
$$

It is well known that the dislocation density tensor and likewise the vectorial measures $\rho^{\mathrm{d}, \boldsymbol{b}}$ suffer from cancellation upon averaging, if an appreciable number of dislocations is present in a volume $V$; in fact, their integrals are supposed to vanish, when the considered volume is large enough compared to the average dislocation spacing. Much more information about the dislocation state of a volume may be captured by introducing further scalar and tensorial (discrete) density measures - which constitute a hierarchy and in principle allow for the recovery of full orientation information of the dislocation tangents in a volume (Hochrainer 2015). We introduce all measures classified by Burgers vector, such that the overall information will typically involve a summation over the Burgers vector bound contributions. The scalar dislocation density measure is defined as

$$
\rho^{\mathrm{d}, \boldsymbol{b}}(\boldsymbol{x})=\sum_{m=1}^{M(\boldsymbol{b})} \int_{c_{m}^{\boldsymbol{b}}} \delta\left(\boldsymbol{x}-\boldsymbol{c}_{m}^{\boldsymbol{b}}(s)\right)\left|\partial_{s} \boldsymbol{c}_{m}^{\boldsymbol{b}}(s)\right| \mathrm{d} s=\sum_{m=1}^{M(\boldsymbol{b})} \int_{c_{m}^{\boldsymbol{b}}} \delta\left(\boldsymbol{x}-\boldsymbol{c}_{m}^{\boldsymbol{b}}(s)\right) \mathrm{d} c,
$$

where $\mathrm{d} c$ denotes the scalar line-element and $\left|\partial_{s} c_{m}^{b}(s)\right|$ is the norm of the tangent, i.e. the 'speed' of the parametrization of the curve.

In order to define the tensorial measures, we introduce the normalized tangent

$$
\boldsymbol{l}_{m}^{\boldsymbol{b}}(s):=\frac{\partial_{s} \boldsymbol{c}_{m}^{\boldsymbol{b}}(s)}{\left|\partial_{s} \boldsymbol{c}_{m}^{\boldsymbol{b}}(s)\right|} .
$$

Moreover, we employ an exponential notation for repeated tensor products of a vector $\boldsymbol{v}$, such that

$$
\boldsymbol{v}^{\otimes n}=\underbrace{\boldsymbol{v} \otimes \ldots \otimes \boldsymbol{v}}_{n-\text { times }}
$$

where we include the understanding that the zeroth tensorial power is defined as $\boldsymbol{v}^{\otimes 0}:=$ 1. With these notations, the distributional alignment tensors of a discrete dislocation distribution are defined as

$$
\boldsymbol{\rho}^{(n) \mathrm{d}, \boldsymbol{b}}(\boldsymbol{x})=\sum_{m=1}^{M(\boldsymbol{b})} \int_{c_{m}^{\boldsymbol{b}}} \delta\left(\boldsymbol{x}-\boldsymbol{c}_{m}^{\boldsymbol{b}}(s)\right) \boldsymbol{l}_{m}^{\boldsymbol{b} \otimes n}(s) \mathrm{d} c .
$$


where the zeroth order alignment tensor is the scalar density, $\boldsymbol{\rho}^{(0) \mathrm{d}, \boldsymbol{b}}=\rho^{\mathrm{d}, \boldsymbol{b}}$, and the first order alignment tensor is the GND vector density, $\boldsymbol{\rho}^{(1) \mathrm{d}, \boldsymbol{b}}=\boldsymbol{\rho}^{\mathrm{d}, \boldsymbol{b}}$.

The total tensorial content of a volume is obtained via integration, which yields

$$
\int_{V} \boldsymbol{\rho}^{(n) \mathrm{d}, \boldsymbol{b}}(\boldsymbol{x}) \mathrm{d} \boldsymbol{x}=\sum_{m=1}^{M(\boldsymbol{b})} \int_{c_{m}^{b} \cap V} \boldsymbol{l}_{m}^{\boldsymbol{b} \otimes n}(s) \mathrm{d} c .
$$

Note that the integral over the scalar discrete density yields the total line length of dislocations in the volume.

For sake of completeness, we note that the full characterization of the dislocation content of a volume also requires two hierarchies of curvature-related tensors in addition to the alignment tensors introduced above (Hochrainer 2015). With the following definition of the dislocation curvature vector,

$$
\boldsymbol{k}_{m}^{\boldsymbol{b}}(s)=\frac{1}{\left|\partial_{s} \boldsymbol{c}_{m}^{\boldsymbol{b}}(s)\right|} \frac{\partial}{\partial s}\left(\boldsymbol{l}_{m}^{\boldsymbol{b}}(s)\right),
$$

the two hierarchies of curvature-related tensors, which do not contain a scalar element (i.e., $n \geq 1$ ), are defined as

$$
\boldsymbol{q}^{(n) \mathrm{d}, \boldsymbol{b}}(\boldsymbol{x})=\sum_{m=1}^{M(\boldsymbol{b})} \int_{c_{m}^{\boldsymbol{b}}} \delta\left(\boldsymbol{x}-\boldsymbol{c}_{m}^{\boldsymbol{b}}(s)\right) \operatorname{sym}\left(\boldsymbol{k}_{m}^{\boldsymbol{b}}(s) \otimes \boldsymbol{l}_{m}^{\boldsymbol{b} \otimes(n-1)}(s)\right) \mathrm{d} c,
$$

and

$$
\boldsymbol{q}^{*(n) \mathrm{d}, \boldsymbol{b}}(\boldsymbol{x})=\sum_{m=1}^{M(\boldsymbol{b})} \int_{c_{m}^{\boldsymbol{b}}} \delta\left(\boldsymbol{x}-\boldsymbol{c}_{m}^{\boldsymbol{b}}(s)\right) \operatorname{sym}\left(\boldsymbol{l}_{m}^{\boldsymbol{b}}(s) \times \boldsymbol{k}_{m}^{\boldsymbol{b}}(s) \otimes \boldsymbol{l}_{m}^{\boldsymbol{b} \otimes(n-1)}(s)\right) \mathrm{d} c .
$$

Here, sym denotes the complete symmetrization of a tensor and $\times$ stands for the crossproduct. We only note that integration of the distributional curvature tensors over a volume likewise turns into integrals over the intersections of the dislocation lines with the volume.

For a volume $V$ with volume content $|V|$, we introduce the average density and curvature tensors of a discrete distribution as

$$
\begin{aligned}
& \overline{\boldsymbol{\rho}}^{(n) \mathrm{d}, \boldsymbol{b}}(V):=\frac{1}{|V|} \int_{V} \boldsymbol{\rho}^{(n) \mathrm{d}, \boldsymbol{b}}(\boldsymbol{x}) \mathrm{d} \boldsymbol{x}, \\
& \overline{\boldsymbol{q}}^{(n) \mathrm{d}, \boldsymbol{b}}(V):=\frac{1}{|V|} \int_{V} \boldsymbol{q}^{(n) \mathrm{d}, \boldsymbol{b}}(\boldsymbol{x}) \mathrm{d} \boldsymbol{x}, \quad \text { and } \\
& \overline{\boldsymbol{q}}^{*(n) \mathrm{d}, \boldsymbol{b}}(V):=\frac{1}{|V|} \int_{V} \boldsymbol{q}^{*(n) \mathrm{d}, \boldsymbol{b}}(\boldsymbol{x}) \mathrm{d} \boldsymbol{x} .
\end{aligned}
$$

So far we considered a single realization for which the discrete densities are obtained. In the sequel, we assume an ensemble of infinitely many realizations, which are labeled using the index $i$. The ensemble averaged dislocation tensors (which do not have the index $\mathrm{d}$ of the discrete single realizations), spatially averaged over a volume $V$, are then defined by

$$
\overline{\boldsymbol{\rho}}^{(n) \boldsymbol{b}}(V)=\left\langle\overline{\boldsymbol{\rho}}^{(n) \mathrm{d}, \boldsymbol{b}}(V)\right\rangle:=\lim _{N \rightarrow \infty} \frac{1}{N} \sum_{i=1}^{N} \overline{\boldsymbol{\rho}}_{i}^{(n) \mathrm{d}, \boldsymbol{b}}(V) .
$$

The definition for average curvature tensors is analogous. We assume that upon ensemble averaging we may obtain smooth densities. These are defined by letting the volumes around each point $\boldsymbol{x}$ go to zero. For convenience, we assume the volumes to be cubes of edge length $a$ with center point $\boldsymbol{x}$, which we denote by $Q_{a}(\boldsymbol{x})$, though the exact shape of 
the (shrinking) volumes does not matter. The pointwise definition of the smooth tensor fields reads ${ }^{1}$

$$
\boldsymbol{\rho}^{(n) \boldsymbol{b}}(\boldsymbol{x})=\lim _{a \rightarrow 0} \overline{\boldsymbol{\rho}}^{(n) \boldsymbol{b}}\left(Q_{a}(\boldsymbol{x})\right)=\lim _{a \rightarrow 0} \lim _{N \rightarrow \infty} \frac{1}{N} \sum_{i=1}^{N} \frac{1}{\left|Q_{a}\right|} \int_{Q_{a}(\boldsymbol{x})} \boldsymbol{\rho}_{i}^{(n) \mathrm{d}, \boldsymbol{b}}(\tilde{\boldsymbol{x}}) \mathrm{d} \tilde{\boldsymbol{x}} .
$$

This definition also transfers to the curvature tensors in an obvious way. If the smooth densities exist, we have

$$
\overline{\boldsymbol{\rho}}^{(n) \boldsymbol{b}}(V)=\frac{1}{|V|} \int_{V} \boldsymbol{\rho}^{(n) \boldsymbol{b}}(\boldsymbol{x}) \mathrm{d} \boldsymbol{x} .
$$

As discussed in more detail in Weger et al. (2021), the discrete volume based average density and curvature tensors may be obtained from straight-segment-based discrete dislocation codes by simple summation formulas. In the current work we provide these formulas without derivation in Section Correlation tensor evaluation from discrete dislocation data.

\section{Pair density tensors}

Since characterizing the dislocation state via single-point quantities already requires the consideration of at least a few low order alignment and curvature tensors, the characterization of correlations between dislocation states at different points necessarily involves tensorial correlation measures. One such type of tensor has already been suggested by Kröner $(1969,2001)$ as part of a series of tensors with higher order correlations. ${ }^{2}$ However, Kröner never fully worked them out or put them to use. Components of the pair density tensor suggested by Kröner have been recently obtained using DDD simulations by Anderson and El-Azab (2021) in the line density approximation, which we will briefly discuss in Section Correlations in 'uncorrelated' distributions of circular loops with the help of Fig. 5. An echo of the tensorial nature can also be noticed in the pair correlations which have been studied in much detail for straight parallel edge dislocations, where correlations of same or opposite orientation dislocations occur, compare (Zaiser et al. 2001). The subsequently defined tensor series has been introduced with slightly different notations and a minor deviation in normalization by Zaiser (2015).

The singular pair density tensors are defined as tensorial distributions on the product space $\Omega \times \Omega$ by

$$
\boldsymbol{\rho}^{\left(n, n^{\prime}\right) \mathrm{d}, \boldsymbol{b}, \boldsymbol{b}^{\prime}}\left(\boldsymbol{x}, \boldsymbol{x}^{\prime}\right)=\left(\sum_{m=1}^{M(\boldsymbol{b})} \int_{c_{m}^{b}} \delta\left(\boldsymbol{x}-\boldsymbol{c}_{m}^{\boldsymbol{b}}(s)\right) \boldsymbol{l}_{m}^{\boldsymbol{b} \otimes n}(s) \mathrm{d} c\right) \otimes\left(\sum_{m^{\prime}=1}^{M\left(\boldsymbol{b}^{\prime}\right)} \int_{c_{m^{\prime}}^{\boldsymbol{b}^{\prime}}} \delta\left(\boldsymbol{x}^{\prime}-\boldsymbol{c}_{m^{\prime}}^{\boldsymbol{b}^{\prime}}(t)\right) \boldsymbol{l}_{m^{\prime}}^{\boldsymbol{b}^{\prime} \otimes n^{\prime}}(t) \mathrm{d} c\right) .
$$

Similar to the proceeding for the single-point tensors, we shall define the smooth ensemble averaged correlation tensors by first introducing the ensemble and spatially averaged correlation tensors for volumes, which in this case means a pair of subvolumes $V$ and $V^{\prime}$. The average correlation tensors of such a volume pair are defined as

\footnotetext{
${ }^{1}$ Note that the ordering of the limits in Eq. (19) may not be exchanged, since for every finite number of realizations, letting the cube size $a$ shrink to zero would recover the constituting delta-distributions.

${ }^{2}$ The two-point correlation tensors of Kröner $(1969,2001)$ are based on the dislocation density tensor Eq. (5) and are therefore most closely related to the vector-vector correlations $\boldsymbol{\rho}^{(1,1) \boldsymbol{b}, \boldsymbol{b}^{\prime}}$, as introduced in Eq. (21).
} 


$$
\overline{\boldsymbol{\rho}}^{\left(n, n^{\prime}\right) \boldsymbol{b}, \boldsymbol{b}^{\prime}}\left(V, V^{\prime}\right)=\left\langle\overline{\boldsymbol{\rho}}^{(n) \mathrm{d}, \boldsymbol{b}}(V) \otimes \overline{\boldsymbol{\rho}}^{\left(n^{\prime}\right) \mathrm{d}, \boldsymbol{b}^{\prime}}\left(V^{\prime}\right)\right\rangle:=\lim _{N \rightarrow \infty} \frac{1}{N} \sum_{i=1}^{N} \overline{\boldsymbol{\rho}}_{i}^{(n) \mathrm{d}, \boldsymbol{b}}(V) \otimes \overline{\boldsymbol{\rho}}_{i}^{\left(n^{\prime}\right) \mathrm{d}, \boldsymbol{b}^{\prime}}\left(V^{\prime}\right) .
$$

The point-wise definition is accordingly

$$
\begin{aligned}
\boldsymbol{\rho}^{\left(n, n^{\prime}\right) \boldsymbol{b}, \boldsymbol{b}^{\prime}}\left(\boldsymbol{x}, \boldsymbol{x}^{\prime}\right) & =\lim _{a \rightarrow 0} \overline{\boldsymbol{\rho}}^{\left(n, n^{\prime}\right) \boldsymbol{b}, \boldsymbol{b}^{\prime}}\left(Q_{a}(\boldsymbol{x}), Q_{a}\left(\boldsymbol{x}^{\prime}\right)\right) \\
& =\lim _{a \rightarrow 0} \lim _{N \rightarrow \infty} \frac{1}{N} \sum_{i=1}^{N} \frac{1}{\left|Q_{a}\right|^{2}} \int_{Q_{a}(\boldsymbol{x})} \boldsymbol{\rho}_{i}^{(n) \mathrm{d}, \boldsymbol{b}}(\tilde{\boldsymbol{x}}) \mathrm{d} \tilde{\boldsymbol{x}} \otimes \int_{Q_{a}\left(\boldsymbol{x}^{\prime}\right)} \boldsymbol{\rho}_{i}^{\left(n^{\prime}\right) \mathrm{d}, \boldsymbol{b}^{\prime}}\left(\tilde{\boldsymbol{x}}^{\prime}\right) \mathrm{d} \tilde{\boldsymbol{x}}^{\prime} .
\end{aligned}
$$

Besides these density-density correlation tensors, there are analogously defined densitycurvature and curvature-curvature correlation tensors, which we only list without argument as

$$
\begin{aligned}
& \boldsymbol{\rho} \boldsymbol{q}^{\left(n, n^{\prime}\right) \boldsymbol{b}, \boldsymbol{b}^{\prime}}=\left\langle\boldsymbol{\rho}^{(n) \mathrm{d}, \boldsymbol{b}} \otimes \boldsymbol{q}^{\left(n^{\prime}\right) \mathrm{d}, \boldsymbol{b}^{\prime}}\right\rangle, \quad \boldsymbol{q} \boldsymbol{\rho}^{\left(n, n^{\prime}\right) \boldsymbol{b}, \boldsymbol{b}^{\prime}}=\left\langle\boldsymbol{q}^{(n) \mathrm{d}, \boldsymbol{b}} \otimes \boldsymbol{\rho}^{\left(n^{\prime}\right) \mathrm{d}, \boldsymbol{b}^{\prime}}\right\rangle, \\
& \boldsymbol{\rho} \boldsymbol{q}^{*\left(n, n^{\prime}\right) \boldsymbol{b}, \boldsymbol{b}^{\prime}}=\left\langle\boldsymbol{\rho}^{(n) \mathrm{d}, \boldsymbol{b}} \otimes \boldsymbol{q}^{*\left(n^{\prime}\right) \mathrm{d}, \boldsymbol{b}^{\prime}}\right\rangle, \quad \boldsymbol{q}^{*} \boldsymbol{\rho}^{\left(n, n^{\prime}\right) \boldsymbol{b}, \boldsymbol{b}^{\prime}}=\left\langle\boldsymbol{q}^{*(n) \mathrm{d}, \boldsymbol{b}} \otimes \boldsymbol{\rho}^{\left(n^{\prime}\right) \mathrm{d}, \boldsymbol{b}^{\prime}}\right\rangle, \\
& \boldsymbol{q} \boldsymbol{q}^{*\left(n, n^{\prime}\right) \boldsymbol{b}, \boldsymbol{b}^{\prime}}=\left\langle\boldsymbol{q}^{(n) \mathrm{d}, \boldsymbol{b}} \otimes \boldsymbol{q}^{*\left(n^{\prime}\right) \mathrm{d}, \boldsymbol{b}^{\prime}}\right\rangle, \quad \boldsymbol{q}^{*} \boldsymbol{q}^{\left(n, n^{\prime}\right) \boldsymbol{b}, \boldsymbol{b}^{\prime}}=\left\langle\boldsymbol{q}^{*(n) \mathrm{d}, \boldsymbol{b}} \otimes \boldsymbol{q}^{\left(n^{\prime}\right) \mathrm{d}, \boldsymbol{b}^{\prime}}\right\rangle, \\
& \boldsymbol{q}^{\left(n, n^{\prime}\right) \boldsymbol{b}, \boldsymbol{b}^{\prime}}=\left\langle\boldsymbol{q}^{(n) \mathrm{d}, \boldsymbol{b}} \otimes \boldsymbol{q}^{\left(n^{\prime}\right) \mathrm{d}, \boldsymbol{b}^{\prime}}\right\rangle, \\
& \boldsymbol{q}^{*\left(n, n^{\prime}\right) \boldsymbol{b}, \boldsymbol{b}^{\prime}}=\left\langle\boldsymbol{q}^{*(n) \mathrm{d}, \boldsymbol{b}} \otimes \boldsymbol{q}^{*\left(n^{\prime}\right) \mathrm{d}, \boldsymbol{b}^{\prime}}\right\rangle .
\end{aligned}
$$

These pair tensor series are unexplored so far and also in the current work we will not go beyond the above formal definition. In the following derivations we shall always focus on pair density and density-density correlations. The transfer of the proceeding to pairings and correlations between densities and curvature or curvature and curvature is in principle obvious.

In stochastics, the deviation of the pair density from being the product of the singlepoint densities is considered as characterizing correlations. The natural definition of the (unscaled) density correlation tensors $\boldsymbol{D}^{\left(n, n^{\prime}\right) \boldsymbol{b}, \boldsymbol{b}^{\prime}}$ consequently is

$$
\boldsymbol{\rho}^{\left(n, n^{\prime}\right) \boldsymbol{b}, \boldsymbol{b}^{\prime}}\left(\boldsymbol{x}, \boldsymbol{x}^{\prime}\right)=\boldsymbol{\rho}^{(n) \boldsymbol{b}}(\boldsymbol{x}) \otimes \boldsymbol{\rho}^{\left(n^{\prime}\right) \boldsymbol{b}^{\prime}}\left(\boldsymbol{x}^{\prime}\right)+\boldsymbol{D}^{\left(n, n^{\prime}\right) \boldsymbol{b}, \boldsymbol{b}^{\prime}}\left(\boldsymbol{x}, \boldsymbol{x}^{\prime}\right) .
$$

The correlation tensors introduced here are not scaled. Note that one may think of different scaling operations, which could for example involve the product of the norms of the single-point tensors. However, this scaling is in general not applicable, because the single-point tensors may vanish, while the two-point density tensors do not, $\mathrm{cf}$. the vector-vector correlations in Section Correlations in 'uncorrelated' distributions of circular loops. We therefore suggest scaling all correlation tensors by the product of the total dislocation densities, such that the scaled correlation tensors are defined as

$$
\boldsymbol{d}^{\left(n, n^{\prime}\right) \boldsymbol{b}, \boldsymbol{b}^{\prime}}\left(\boldsymbol{x}, \boldsymbol{x}^{\prime}\right)=\frac{1}{\rho^{\boldsymbol{b}}(\boldsymbol{x}) \rho^{\boldsymbol{b}^{\prime}\left(\boldsymbol{x}^{\prime}\right)}} \boldsymbol{D}^{\left(n, n^{\prime}\right) \boldsymbol{b}, \boldsymbol{b}^{\prime}}\left(\boldsymbol{x}, \boldsymbol{x}^{\prime}\right) .
$$

While these correlations are thus expected to be (upon appropriately scaling the space coordinates) independent of the total density, they will not be invariant with respect to the single-point tensors involved. Because of this open scaling issue, we shall mostly concentrate on the unscaled correlation tensors in the current work.

Moreover, we note that in dislocation systems we cannot employ the criterion that an uncorrelated system would imply vanishing density correlation tensors $\boldsymbol{D}^{\left(n, n^{\prime}\right) \boldsymbol{b}, \boldsymbol{b}^{\prime}}=\mathbf{0}$. 
A random distribution of oriented, disconnected, straight segments could in principle be completely uncorrelated; however, segments which are part of connected curves are necessarily strongly correlated along the line, as will be discussed in detail in the next section. Theoretically one may separate correlations from the same dislocation from the contributions of other dislocations when analyzing DDD data. However, there are several reasons why this does not appear to be a practical way of separation: a) in actual dislocation configurations there are typically no well-distinguishable closed curves, but at most segments which may be clearly assigned to "the same" curve, b) at larger distances, the fact that two segments belong to the same dislocation (if this assignment is possible) might be irrelevant and elastic interactions prevail, and c) in doing practical evaluations from DDD simulations, it seems a disproportional effort to group the dislocation segments accordingly before evaluating the tensors. We therefore suggest to investigate the consequences of the connectivity at simple example distributions in order to be able to identify the typical self-correlation from averaged results from DDD simulations. Before studying connectivity in distributions of circular loops in Section Correlations in 'uncorrelated' distributions of circular loops, however, we shall devote the next subsection to the consequences of assuming spatial invariance, which entails that all two-point quantities only depend on the distance vector between two points.

\section{Correlations in distance space}

Real materials will be finite, usually polycrystalline and in general containing further defects, which will make typical dislocation distributions explicitly space dependent. However, if crystals are large enough as compared to the average dislocation spacing and the concentrations of other defects are low and statistically homogeneous, assuming dislocation systems without explicit space dependence seems to be reasonable for studying dislocation correlations. For DDD simulations, this will typically mean studying periodic systems. Spatial invariance, i.e., translational symmetry on the one hand implies that all single-point ensemble averaged (!) quantities are actually constant in space. On the other hand, all pair densities and correlations consequently only depend on the distance vector $\boldsymbol{r}=\boldsymbol{x}^{\prime}-\boldsymbol{x}$ between two points.

Single realizations are of course not spatially homogeneous and one applies spatial averaging to map the discrete densities into homogeneous quantities. Let $\Omega$ be the periodic unit cell, then the homogenized single-point and two-point density tensors of a single realization are given by

$$
\begin{aligned}
\boldsymbol{\rho}^{(n) \mathrm{d}, \boldsymbol{b}} & =\frac{1}{|\Omega|} \int_{\Omega} \boldsymbol{\rho}^{(n) \mathrm{d}, \boldsymbol{b}}(\boldsymbol{x}) d \boldsymbol{x}=\overline{\boldsymbol{\rho}}^{(n) \mathrm{d}, \boldsymbol{b}}(\Omega), \quad \text { and } \\
\boldsymbol{\rho}^{\left(n, n^{\prime}\right) \mathrm{d}, \boldsymbol{b}, \boldsymbol{b}^{\prime}}(\boldsymbol{r}) & =\frac{1}{|\Omega|} \int_{\Omega} \boldsymbol{\rho}^{\left(n, n^{\prime}\right) \mathrm{d}, \boldsymbol{b}, \boldsymbol{b}^{\prime}}(\boldsymbol{x}, \boldsymbol{x}+\boldsymbol{r}) d \boldsymbol{x},
\end{aligned}
$$

where we note that the plus sign in the argument in the second line must of course respect periodicity. The single-point tensors accordingly yield global values and ensemble averaging over the realizations yields the average tensors of the ensemble,

$$
\boldsymbol{\rho}^{(n) \boldsymbol{b}}=\left\langle\boldsymbol{\rho}^{(n) \mathrm{d}, \boldsymbol{b}}\right\rangle .
$$

Note that the linearity of both, spatial and ensemble averaging, implies that these operations commute. That means, for instance, that the ensemble average of the homogenized 
pair density equals the homogenized ensemble averaged density,

$$
\boldsymbol{\rho}^{\left(n, n^{\prime}\right) \boldsymbol{b}, \boldsymbol{b}^{\prime}}(\boldsymbol{r})=\left\langle\boldsymbol{\rho}^{\left(n, n^{\prime}\right) \mathrm{d}, \boldsymbol{b}, \boldsymbol{b}^{\prime}}(\boldsymbol{r})\right\rangle=\frac{1}{|\Omega|} \int_{\Omega} \boldsymbol{\rho}^{\left(n, n^{\prime}\right) \boldsymbol{b}, \boldsymbol{b}^{\prime}}(\boldsymbol{x}, \boldsymbol{x}+\boldsymbol{r}) d \boldsymbol{x} .
$$

When evaluating spatial correlations in supposedly translationally invariant data, one likewise usually evaluates already the correlations from single realizations in (a discretized) distance space. This is also done in the current work, as detailed in Section Numerical schemes to evaluate correlations from line data. However, in the case of curves or curved segments (or any other extended objects for that matter), the projection of the singular pair density data into distance space features a peculiarity, which will play a vital role for understanding self-correlations in Section Correlations in 'uncorrelated' distributions of circular loops. Therefore, we shall devote the rest of this subsection to analyzing the pair density information induced by curves in the space of distance vectors, which we shall usually call the distance space in the sequel.

We begin by inserting the integral definition of the singular densities into the definition of the singular density tensors of a pair of curves $c_{m}^{b}, c_{m^{\prime}}^{\boldsymbol{b}^{\prime}}$ in product space. This yields

$$
\begin{aligned}
\boldsymbol{\rho}_{m, m^{\prime}}^{\left(n, n^{\prime}\right) \mathrm{d}, \boldsymbol{b}, \boldsymbol{b}^{\prime}}\left(\boldsymbol{x}, \boldsymbol{x}^{\prime}\right) & =\int_{c_{m}^{\boldsymbol{b}}} \delta\left(\boldsymbol{x}-\boldsymbol{c}_{m}^{\boldsymbol{b}}(s)\right) \boldsymbol{l}_{m}^{\boldsymbol{b} \otimes n}(s) \mathrm{d} c \otimes \int_{c_{m^{\prime}}^{\boldsymbol{b}^{\prime}}} \delta\left(\boldsymbol{x}^{\prime}-\boldsymbol{c}_{m^{\prime}}^{\boldsymbol{b}^{\prime}}(t)\right) \boldsymbol{l}_{m^{\prime}}^{\boldsymbol{b}^{\prime} \otimes n^{\prime}}(t) \mathrm{d} c^{\prime} \\
& =\int_{c_{m}^{\boldsymbol{b}} \times c_{m^{\prime}}^{\boldsymbol{b}^{\prime}}} \delta\left(\left(\boldsymbol{x}, \boldsymbol{x}^{\prime}\right)-\left(\boldsymbol{c}_{m}^{\boldsymbol{b}}(s), \boldsymbol{c}_{m^{\prime}}^{\boldsymbol{b}^{\prime}}(t)\right)\right) \boldsymbol{l}_{m}^{\boldsymbol{b} \otimes n}(s) \otimes \boldsymbol{l}_{m^{\prime}}^{\boldsymbol{b}^{\prime} \otimes n^{\prime}}(t)\left|\partial_{s} \boldsymbol{c}_{m}^{\boldsymbol{b}}(s) \| \partial_{t} \boldsymbol{c}_{m^{\prime}}^{\boldsymbol{b}^{\prime}}(t)\right| \mathrm{d} s \mathrm{~d} t \\
& =\int_{c_{m}^{\boldsymbol{b}} \times c_{m^{\prime}}^{\boldsymbol{b}^{\prime}}} \delta\left(\left(\boldsymbol{x}, \boldsymbol{x}^{\prime}\right)-\left(\boldsymbol{c}_{m}^{\boldsymbol{b}}(s), \boldsymbol{c}_{m^{\prime}}^{\boldsymbol{b}^{\prime}}(t)\right)\right) \boldsymbol{l}_{m}^{\boldsymbol{b} \otimes n}(s) \otimes \boldsymbol{l}_{m^{\prime}}^{\boldsymbol{b}^{\prime} \otimes n^{\prime}}(t) \mathrm{d} A_{\times}
\end{aligned}
$$

where $c_{m}^{\boldsymbol{b}} \times c_{m^{\prime}}^{\boldsymbol{b}^{\prime}}$ denotes the two-dimensional product manifold generated by the two curves in product space and

$$
\mathrm{d} A_{\times}=\left|\partial_{s} \boldsymbol{c}_{m}^{\boldsymbol{b}}(s)\right|\left|\partial_{t} \boldsymbol{c}_{m^{\prime}}^{\boldsymbol{b}^{\prime}}(t)\right| \mathrm{d} s \mathrm{~d} t
$$

is the scalar surface element on this product manifold.

The homogenized version of Eq. (34) is found as

$$
\begin{aligned}
\boldsymbol{\rho}_{m, m^{\prime}}^{\left(n, n^{\prime}\right) \mathrm{d}, \boldsymbol{b}, \boldsymbol{b}^{\prime}}(\boldsymbol{r}) & =\frac{1}{|\Omega|} \int_{\Omega} \boldsymbol{\rho}_{m, m^{\prime}}^{\left(n, n^{\prime}\right) \mathrm{d}, \boldsymbol{b}, \boldsymbol{b}^{\prime}}(\boldsymbol{x}, \boldsymbol{x}+\boldsymbol{r}) d \boldsymbol{x} \\
& =\frac{1}{|\Omega|} \int_{I} \int_{I^{\prime}} \delta\left(\boldsymbol{r}-\left(\boldsymbol{c}_{m^{\prime}}^{\boldsymbol{b}^{\prime}}(t)-\boldsymbol{c}_{m}^{\boldsymbol{b}}(s)\right)\right) \boldsymbol{l}_{m}^{\boldsymbol{b} \otimes n}(s) \otimes \boldsymbol{l}_{m^{\prime}}^{\boldsymbol{b}^{\prime} \otimes n^{\prime}}(t)\left|\partial_{s} \boldsymbol{c}_{m}^{\boldsymbol{b}}(s) \| \partial_{t} \boldsymbol{c}_{m^{\prime}}^{\boldsymbol{b}^{\prime}}(t)\right| \mathrm{d} s \mathrm{~d} t
\end{aligned}
$$

where $I$ and $I^{\prime}$ are intervals of parametrizations of $c_{m}^{\boldsymbol{b}}$ and $c_{m^{\prime}}^{\boldsymbol{b}^{\prime}}$, respectively. We note that this derivation involves the product of two $\delta$-distributions which share the integration variable as arguments, which is not in general defined. The formal justification for (the quite intuitive) identity in Eq. (37) is therefore provided in Appendix A.

Note, moreover, that we introduced intervals of parametrization in Eq. (37) but not in (34). The reason is that there is a difference between the single-point density in Eq. (10) and the two-point density in product space in (32) on the one hand and the density in difference space in (37) on the other hand. The single-point densities adding up to $\boldsymbol{\rho}^{(n) \mathrm{d}, \boldsymbol{b}}(\boldsymbol{x})$ are concentrated by virtue of a unit measure on the one-dimensional submanifolds of the dislocation lines, as may be seen from the occurrence of the scalar line-elements $\mathrm{d} c$ in the integral definitions. Likewise, the two-point density $\boldsymbol{\rho}_{m, m^{\prime}}^{\left(n, n^{\prime}\right) \mathrm{d}, \boldsymbol{b}, \boldsymbol{b}^{\prime}}\left(\boldsymbol{x}, \boldsymbol{x}^{\prime}\right)$ is concentrated by virtue of a unit measure on the two-dimensional submanifold spanned by 
the two curves in product space, as the integral involves the scalar surface element $\mathrm{d} A_{\times}$ on the product manifold. The discrete density $\boldsymbol{\rho}_{m, m^{\prime}}^{\left(n, n^{\prime}\right) \mathrm{d}, \boldsymbol{b}, \boldsymbol{b}^{\prime}}(\boldsymbol{r})$ on distance space, by contrast, is concentrated on the space of distance vectors between the curves, which is firstly not a two-dimensional submanifold but just an immersion of the two-dimensional parametrization space into $\mathbb{R}^{3}$, which will in general contain intersections and overlaps. Secondly, the density is not concentrated on this immersion as a unit measure, because at points where the set is a surface (note that pairs of parallel curve segments map to lines rather than surfaces; a case we shall discuss in Appendix B), the surface element in the natural parametrization inherited by the two curves is

$$
d A=\left|\partial_{s} \boldsymbol{c}_{m}^{\boldsymbol{b}}(s) \times \partial_{t} \boldsymbol{c}_{m^{\prime}}^{\boldsymbol{b}^{\prime}}(t)\right| \mathrm{d} s \mathrm{~d} t .
$$

In order to derive the weighting function needed to quantify the deviation from being the unit measure on the set of distance vectors we introduce the projection

$$
\begin{aligned}
\Pi: \mathbb{R}^{3} \times \mathbb{R}^{3} & \rightarrow \mathbb{R}^{3} \\
\left(\boldsymbol{x}, \boldsymbol{x}^{\prime}\right) & \mapsto \Pi\left(\boldsymbol{x}, \boldsymbol{x}^{\prime}\right)=\boldsymbol{r}\left(\boldsymbol{x}, \boldsymbol{x}^{\prime}\right)=\boldsymbol{x}^{\prime}-\boldsymbol{x},
\end{aligned}
$$

from product to distance space. The mapping $\Pi$ will in general immerse the twodimensional submanifold spanned by two curve segments $c_{m}^{\boldsymbol{b}}$ and $c_{m^{\prime}}^{\boldsymbol{b}^{\prime}}$ in $\mathbb{R}^{3}$ with overlaps and intersections. However, if the two regarded curve segments $c_{m}^{\boldsymbol{b}}$ and $c_{m^{\prime}}^{\boldsymbol{b}^{\prime}}$ are short enough and not parallel to each other on finite intervals, the image $\Pi\left(c_{m}^{\boldsymbol{b}} \times c_{m^{\prime}}^{\boldsymbol{b}^{\prime}}\right)$ is likewise a two-dimensional submanifold of $\mathbb{R}^{3}$, that is, a surface. The discrete pair density of such segments is then given by an integral over this submanifold in distance space as

$$
\boldsymbol{\rho}_{m, m^{\prime}}^{\left(n, n^{\prime}\right) \mathrm{d}, \boldsymbol{b}, \boldsymbol{b}^{\prime}}(\boldsymbol{r})=\frac{1}{|\Omega|} \int_{\Pi\left(c_{m}^{\boldsymbol{b}} \times c_{m^{\prime}}^{\boldsymbol{b}^{\prime}}\right)} \phi_{m, m^{\prime}}^{\boldsymbol{b}, \boldsymbol{b}^{\prime}}(s, t) \delta\left(\boldsymbol{r}-\left(\boldsymbol{c}_{m^{\prime}}^{\boldsymbol{b}^{\prime}}(t)-\boldsymbol{c}_{m}^{\boldsymbol{b}}(s)\right)\right) \boldsymbol{l}_{m}^{\boldsymbol{b} \otimes n}(s) \otimes \boldsymbol{l}_{m^{\prime}}^{\boldsymbol{b}^{\prime} \otimes n^{\prime}}(t) \mathrm{d} A,
$$

with

$$
\phi_{m, m^{\prime}}^{\boldsymbol{b}, \boldsymbol{b}^{\prime}}(s, t)=\frac{\left|\partial_{s} \boldsymbol{c}_{m}^{\boldsymbol{b}}(s)\right|\left|\partial_{t} \boldsymbol{c}_{m^{\prime}}^{\boldsymbol{b}^{\prime}}(t)\right|}{\left|\partial_{s} \boldsymbol{c}_{m}^{\boldsymbol{b}}(s) \times \partial_{t} \boldsymbol{c}_{m^{\prime}}^{\boldsymbol{b}^{\prime}}(t)\right|}=\frac{1}{\left|\boldsymbol{l}_{m}^{\boldsymbol{b}}(s) \times \boldsymbol{l}_{m^{\prime}}^{\boldsymbol{b}^{\prime}}(t)\right|}=\frac{1}{\left|\sin \left(\alpha_{m, m^{\prime}}^{\boldsymbol{b}, \boldsymbol{b}^{\prime}}(s, t)\right)\right|} .
$$

Here, $\alpha_{m, m^{\prime}}^{\boldsymbol{b}, \boldsymbol{b}^{\prime}}(s, t)$ denotes the angle between the local tangents of the curves.

If the distance vectors between two curve segments do not map to a submanifold in distance space, we assume that we can separate the defining parametrization space into subsets which map into non-intersecting submanifolds. However, while we ignore intersections because they are of a lower dimensionality and thus do not matter upon integration, it may be that these submanifolds in distance space overlap in parts or in whole. Overlaps will especially occur for planar curves. In the case of overlaps, the function $\phi_{m, m^{\prime}}^{\boldsymbol{b}, \boldsymbol{b}^{\prime}}(s, t)$ and likewise the unit tangents $\boldsymbol{l}_{m}^{\boldsymbol{b}}$ and $\boldsymbol{l}_{m^{\prime}}^{\boldsymbol{b}^{\prime}}$, may be multivalued at points in the subset $\Pi\left(c_{m}^{\boldsymbol{b}} \times c_{m^{\prime}}^{\boldsymbol{b}^{\prime}}\right)$ of distance space.

We assume that for every pair of curves $\left(c_{m}^{\boldsymbol{b}}, c_{m^{\prime}}^{\boldsymbol{b}^{\prime}}\right)$ in a realization we can decompose the parametrization area $I \times I^{\prime} \in \mathbb{R}^{2}$ into $k \cdot k^{\prime}$ rectangular regions $I_{i} \times I_{j}^{\prime}$, which tile the total parametrization area,

$$
\bigcup_{\substack{i=1, \ldots, k \\ j=1, \ldots, k^{\prime}}} I_{i} \times I_{j}^{\prime}=I \times I^{\prime}
$$


and such that the map

$$
\begin{aligned}
H_{m, m^{\prime}}^{\boldsymbol{b}, \boldsymbol{b}^{\prime}}: I \times I^{\prime} & \rightarrow \mathbb{R}^{3} \\
(s, t) & \mapsto H_{m, m^{\prime}}^{\boldsymbol{b}, \boldsymbol{b}^{\prime}}(s, t):=\Pi\left(\boldsymbol{c}_{m}^{\boldsymbol{b}}(s), \boldsymbol{c}_{m^{\prime}}^{\boldsymbol{b}^{\prime}}(t)\right)=\boldsymbol{c}_{m^{\prime}}^{\boldsymbol{b}^{\prime}}(t)-\boldsymbol{c}_{m}^{\boldsymbol{b}}(s),
\end{aligned}
$$

is bijective when restricted to each region $I_{i} \times I_{j}^{\prime}$. Requiring bijectivity means that the images of the regions are two-dimensional submanifolds, which in turn means that for this assumption to hold we need to exclude pairs of dislocations (including the pairing of a single dislocation with itself) where there exist two non-degenerate intervals $\left[s_{1}, s_{2}\right) \in I$ and $\left[t_{1}, t_{2}\right) \in I^{\prime}$ such that the tangents of the curves are constant and parallel, i.e., $\boldsymbol{k}_{m}^{\boldsymbol{b}}(s)=\boldsymbol{k}_{m^{\prime}}^{\boldsymbol{b}^{\prime}}(t)=0$ and $\boldsymbol{c}_{m}^{\boldsymbol{b}}(s) \| \boldsymbol{c}_{m^{\prime}}^{\boldsymbol{b}^{\prime}}(t)$ for all $(s, t) \in\left[s_{1}, s_{2}\right) \times\left[t_{1}, t_{2}\right)$. Because dislocations do actually tend to be straight, we discuss this case for sake of completeness in Appendix B.

Even upon excluding parallel segments, the image of $H$ may contain intersections and overlaps, such that different parameters $(s, t) \neq(u, v)$ may map to the same point, i.e.

$$
H_{m, m^{\prime}}^{\boldsymbol{b}, \boldsymbol{b}^{\prime}}(s, t)=H_{m, m^{\prime}}^{\boldsymbol{b}, \boldsymbol{b}^{\prime}}(u, v)=\boldsymbol{r} .
$$

Consequently, at the same point $\boldsymbol{r}$ in the image in distance space the function $\phi_{m, m^{\prime}}^{\boldsymbol{b}, \boldsymbol{b}^{\prime}}(s, t)$ and the tangents may take several values, depending on the point pairs which share the same distance vector. For all regarded points $r \in \mathbb{R}^{3}$ we assume that there are only a finite number of parameter pairs which map to this point. This excludes, for instance, the origin in the case when both curves are the same or a shift vector which would map two dislocations or finite segments of them upon each other by a rigid body translation. Let now $\boldsymbol{r}$ be in the image of $H$ and $H^{-1}(\boldsymbol{r}) \subset I \times I^{\prime}$ is the finite set of each time two parameters yielding the same distance vector. In this case we define the function $\phi(r)$ by the sum

$$
\phi_{m, m^{\prime}}^{\boldsymbol{b}, \boldsymbol{b}^{\prime}}(\boldsymbol{r})=\sum_{(s, t) \in\left(H_{m, m^{\prime}}^{\boldsymbol{b}, \boldsymbol{b}^{\prime}}\right)^{-1}(\boldsymbol{r})} \phi_{m, m^{\prime}}^{\boldsymbol{b}, \boldsymbol{b}^{\prime}}(s, t) .
$$

Because the multivaluedness extends to the unit tangents occurring in the tensorial definitions, we likewise define a tensorial function on the parametrization space by

$$
\boldsymbol{\phi}_{m, m^{\prime}}^{\boldsymbol{b}, \boldsymbol{b}^{\prime}\left(n, n^{\prime}\right)}(s, t)=\boldsymbol{\phi}_{m, m^{\prime}}^{\boldsymbol{b}, \boldsymbol{b}^{\prime}}(s, t) \boldsymbol{l}_{m}^{\boldsymbol{b} \otimes n}(s) \otimes \boldsymbol{l}_{m^{\prime}}^{\boldsymbol{b}^{\prime} \otimes n^{\prime}}(t)
$$

The spatial version of this on the image of $H$ is accordingly defined by

$$
\boldsymbol{\phi}_{m, m^{\prime}}^{\boldsymbol{b}, \boldsymbol{b}^{\prime}\left(n, n^{\prime}\right)}(\boldsymbol{r})=\sum_{(s, t) \in\left(H_{m, m^{\prime}}^{\boldsymbol{b}, \boldsymbol{b}^{\prime}}\right)^{-1}(\boldsymbol{r})} \boldsymbol{\phi}_{m, m^{\prime}}^{\boldsymbol{b}, \boldsymbol{b}^{\prime}\left(n, n^{\prime}\right)}(s, t) .
$$

In the following section these tensor functions play a key role for deriving selfcorrelation tensors in systems of otherwise uncorrelated circles.

\section{Correlations in 'uncorrelated' distributions of circular loops}

The key challenges in understanding dislocation correlations are their tensorial character and the fact that the line-like nature and connectivity of dislocations implies spatial correlations along the dislocation line even if the positions of dislocation segments would not be correlated due to their elastic interactions. As will be discussed later in more detail, the correlations due to connectivity are relevant for a continuum theory, but we think it 
is important to distinguish them from the 'elastic' correlations when analysing dislocation correlation data. Correlations related to connectivity shall be called self-correlations ${ }^{3}$ in the sequel and we speak of pair correlations for those resulting from elastic interactions. In the current section we exclude pair correlations and solely concentrate on self-correlations in artificial ensembles of curves. Before we proceed, we note, anticipating some results obtained below, that a complete distinction between connectivity-based and elastic correlations is in general not possible. For example, the average dislocation curvature is reflected in self-correlations, while it may likewise reflect an average stress state. We believe nevertheless that it is instructive to determine self-correlations in systems of otherwise uncorrelated systems of dislocations for two reasons: on the one hand, this is important for assessing their relative contribution in numerically obtained correlation data. On the other hand, because we may derive such correlations analytically, it is also instructive for understanding the content and interpretation of the pair correlation tensors per se.

Self-correlations in systems of random curves have been studied first by Stoyan and Stoyan (1986). This work has received little attention in the dislocation community and its consequences were only addressed in one (Csikor et al. 2007) of the few studies of correlations in general dislocation systems. The work by Stoyan and Stoyan (1986) contains analytical results on correlations for various artificial dislocation distributions, e.g. random straight lines, random circles or pairs of either. However, that work is limited to correlations of total dislocation density at two points, i.e., $\rho^{(0,0)}$ or $d^{(0,0)}$ in our terminology. In the current section we first present the derivation of Stoyan and Stoyan (1986) for loops of a given radius and then provide the generalization of these results to correlation tensors of higher order. This generalization will first be done in the spirit of Stoyan and Stoyan (1986), before we provide the general case based on the weighting function $\phi$ and the tensors $\phi^{\left(n, n^{\prime}\right)}$ introduced in the preceding Section Correlations in distance space. We shall assume homogeneous systems and because the following considerations are just based on the geometry of curves, we leave out the Burgers vector classification in the notation of this section.

In Stoyan and Stoyan (1986) the pair density $\rho^{(0,0)}(\boldsymbol{r})$, i.e., the probability to find dislocation segments simultaneously at two points with distance vector $\boldsymbol{r}$, is constructed as the product of the probability to find a dislocation at some point (serving as the origin $\mathbf{0}$ in distance space) and the conditional probability to find a point on a dislocation the distance vector $\boldsymbol{r}$ apart, knowing that there is a dislocation at $\mathbf{0}$. The latter conditional probability shall be denoted as $\rho(\boldsymbol{r} \mid$ disl. at $\mathbf{0})$. The probability to find a dislocation 'at $\mathbf{0}$ ' is the single-point density $\rho(\boldsymbol{r}) \equiv \rho(\mathbf{0})=\rho$, such that the pair density results as

$$
\rho^{(0,0)}(\boldsymbol{r})=\rho \cdot \rho(\boldsymbol{r} \mid \text { disl. at } \mathbf{0}) \text {. }
$$

For uncorrelated systems of circles or straight lines, the pair probability $\rho^{(0,0)}(\boldsymbol{r})$ can be calculated analytically. In these cases, the pair density is rotationally invariant and the pair density only depends on $\boldsymbol{r}$ via the modulus $r=|\boldsymbol{r}|$,

$$
\rho^{(0,0)}(\boldsymbol{r})=\rho^{(0,0)}(r) \text {. }
$$

\footnotetext{
${ }^{3}$ Note that this deviates from the use of this term in Anderson and El-Azab (2021), where this refers to correlations between dislocations of the same slip system. Both definitions coincide, though, for the specific DDD configurations analyzed in Section Correlation tensor evaluation from discrete dislocation data.
} 
We shall concentrate on the planar case in the sequel and assume a distribution of circles with fixed radius $R$. For the three-dimensional case we refer to Stoyan and Stoyan (1986). The treatment of a distribution of radii will be briefly discussed in Section Correlations in homogeneous systems with a distribution of radii. Because we deal with a planar case here, minor adaptions will be necessary when applying the results to the three-dimensional data in Section Correlation tensor evaluation from discrete dislocation data.

The ensemble we look at are circles with fixed radius $R$ with homogeneously distributed random centerpoints in the plane. The centerpoints are assumed to be generated by a two-dimensional Poisson point process with density $\rho_{\mathrm{c}}$. The dislocation density is accordingly given by $\rho=2 \pi R \rho_{c}$. The scalar curvature density $q$ of the planar tensor expansion (compare Hochrainer et al. (2014); Hochrainer (2015)) depends on specifying the orientation of the loops, which fixes the scalar curvature to be $k= \pm 1 / R$, where the positive sign holds for positively (counter clockwise) oriented loops. If both orientations occur with the same probability, the average scalar curvature density vanishes. If all loops have positive orientation, the scalar curvature density is given by $q=k \rho=\frac{\rho}{R}=2 \pi \rho_{\mathrm{c}}$. We note in passing that this illustrates the interpretation of $q$ as a kind of number density of dislocations, which also holds for general loop shapes, cf. Hochrainer (2015); Weger et al. (2021). In the regarded ensembles the dislocation density vector and all other alignment tensors of odd degree vanish irrespective of the distribution of orientations. The irreducible (i.e. traceless, compare Hochrainer (2015)) components of all higher order alignment tensors of even degree likewise vanish, such that the reducible alignment tensors may all be determined from the (zeroth order) density $\rho$. Moreover, also all higher order irreducible curvature tensors vanish irrespective of the distribution of orientations. Before we recall the derivation of the pair density for such an ensemble as provided by Stoyan and Stoyan (1986), we shall briefly give an introduction to Fig. 1, which is of vital relevance for the considerations in this section.

Figure 1 displays three circles, two red circles of the given radius $R$ and a blue circle with a general radius $r<2 R$. The bold red circle is a 'typical (dislocation) loop' of radius $R$ which crosses the origin in distance space. The point on the red circle at the origin is considered a 'typical point' on a loop. The tangent to the origin includes the angle $\omega$ with the $x$-direction. The intersection point of the blue circle and the bold red circle together with the point at the origin is considered as a pair of points which both lie on the bold red circle. The intersection point of the circles has polar coordinates $\varphi$ and $r$, where $r$ defines the radius of the blue circle. The thin red circle indicates that there is another pair of points on a circle of radius $R$ with the same distance vector $r$. Further explanations on other quantities shown in Fig. 1 will be given where needed.

The approach in Stoyan and Stoyan (1986) starts from the integral of the pair density in a sphere or - in the planar case - in a circular disk around a 'typical point' on a dislocation. The blue area in Fig. 1 is such a circular disk with radius $r$. The integral of the pair density in an area is the expected total line length in the area. In the considered planar case, we expect to find in a circular disc of radius $r$ around a point on a dislocation $i$ ) the same line length like in a circular disc around any other point, which follows from the constant 


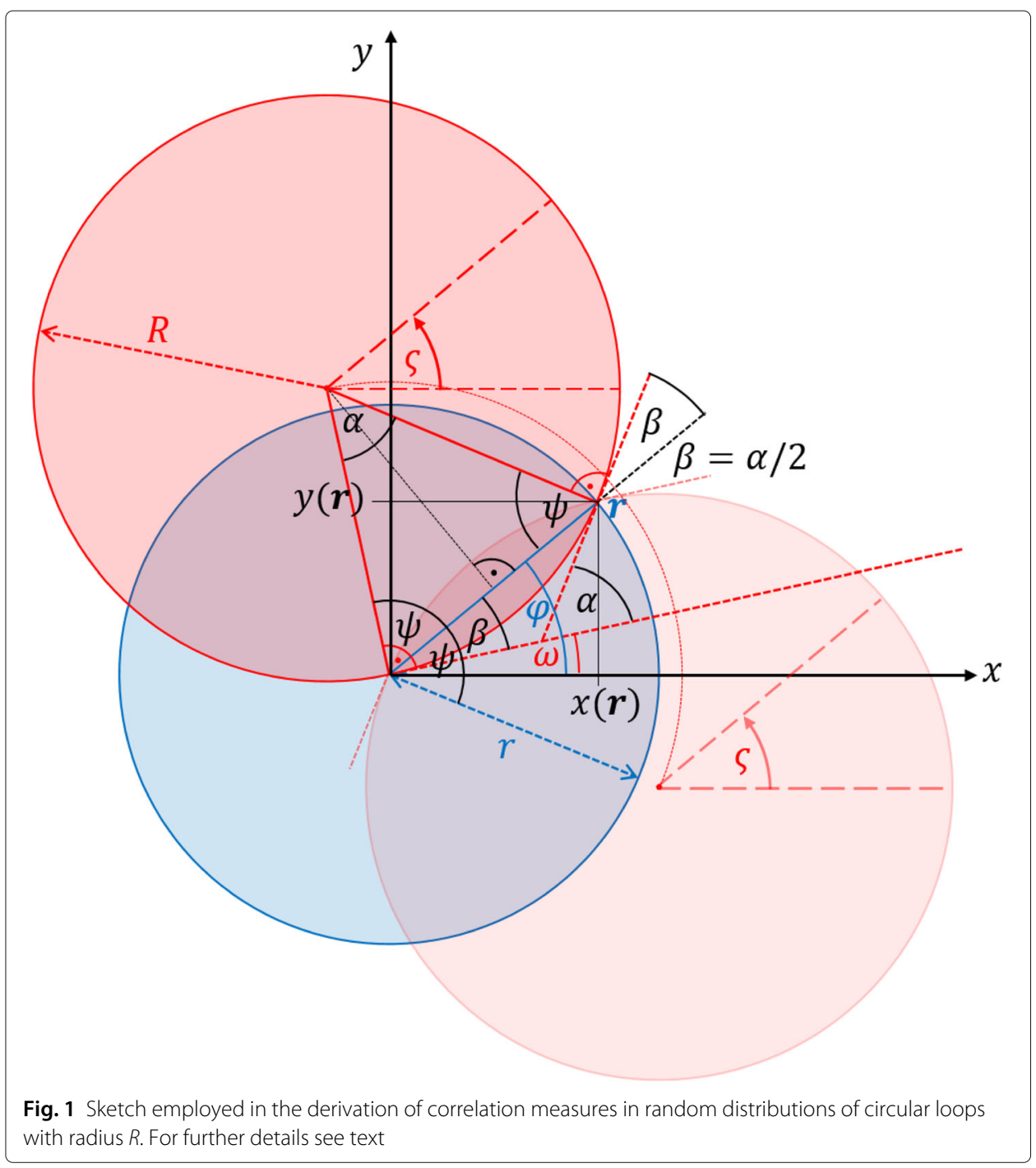

background density ${ }^{4}$, i.e. $\pi r^{2} \rho$, plus $i i$ ) the line length of the intersection with the circular disc of the dislocation the point is located on. The latter is the length of the intersection of a circle of radius $R$ through the origin with the circular disc of radius $r$, as depicted in Fig. 1. The angle corresponding to the intersecting red circle segment is twice the angle $\alpha$ shown in Fig. 1. As may be read from the red-blue triangle in Fig. 1, this angle is given as a function of $r$ (and $R$, which we assume fixed) by

$$
\alpha(r)=2 \arcsin \frac{r}{2 R} \text {. }
$$

The expected line length in a circle of radius $r$ around a typical point on a loop contains two segments with angular range $\alpha(r)$, such that the average line length in a circle of radius $r$ around a point on a dislocation reads

$$
L(r)=\pi r^{2} \rho+4 R \arcsin \frac{r}{2 R} .
$$

\footnotetext{
${ }^{4}$ In finite systems one has to consider that the background density does not contain the very loop the point is located on. This is considered in Section Correlation tensor evaluation from discrete dislocation data, but we ignore it in the current section as was done by Stoyan and Stoyan (1986).
} 
The expected dislocation density at distance $r$ from the center is given by the line length increase per increase in radius $r$ of the circular disc, divided by the length of the circle of radius $r$, i.e.

$$
\rho(r \mid \text { disl. at } \mathbf{0})=\frac{1}{2 \pi r} \frac{\mathrm{d} L(r)}{\mathrm{d} r}=\rho+\frac{1}{\pi r \sqrt{1-r^{2} /\left(4 R^{2}\right)}} .
$$

The pair density is accordingly found as

$$
\rho^{(0,0)}(\boldsymbol{r})=\rho^{2}+\frac{\rho}{\pi r \sqrt{1-r^{2} /\left(4 R^{2}\right)}},
$$

and the correlation function is

$$
d^{(0,0)}(\boldsymbol{r})=\frac{1}{\rho \pi r \sqrt{1-r^{2} /\left(4 R^{2}\right)}} .
$$

For $r>2 R$ the correlation function and the according term in Eq. (55) vanish. The correlations diverge both at the origin, for $r \rightarrow 0$, as when $r$ approaches the diameter of the circles, i.e. for $r \rightarrow 2 R$. As shown in Section Correlations in homogeneous systems with a distribution of radii the divergence around the center, which is of type $1 / r$, is generic for planar dislocation distributions, while the singularity at the diameter is an artifact of the fixed radius of the circle distribution. Plots of the pair correlation function are provided in Section Correlations in homogeneous systems with a distribution of radii, Fig. 9b and c, where it is compared to correlation functions of distributions with varying radii, and plots of the pair density function are provided in Section Validation: self-correlations of randomly distributed loops with constant radius, as contour plot in Fig. 10e and as line plot in Fig. 11, where the pair density was obtained (in slightly modified form) from numerical averaging over realizations of random circular loops.

A similar reasoning as for the total density correlations may be applied to obtain the pair correlation vector field $\boldsymbol{D}^{(0,1)}$, which captures the correlation between a point where there is a dislocation of unknown line orientation and the expected GND vector field around it. We note that the correlated GND vector field occurs in a GND-free $(\rho=0)$ distribution, such that $\boldsymbol{D}^{(0,1)}=\rho^{(0,1)}$. We again start from an integral reasoning; this time from modeling the probability of a point $\boldsymbol{r}$ to be encircled by a loop through the origin. Also this probability function, which we denote with $\zeta(\boldsymbol{r} \mid$ disl. at $\mathbf{0})$ only depends on the distance $r$ from the origin. In terms of dislocations, this function is the conditional probability -knowing that there is a loop of radius $R$ through the origin- of a point to have been plastically sheared by the loop, presuming that the loops are the boundaries of swept circular discs. Before providing this conditional probability, we highlight how this function relates to the sought correlation vector field $D^{(0,1)}$.

For a single slip system with Burgers vector $\boldsymbol{b}$ and normal $\boldsymbol{n}$ the plastic distortion is defined by an according $\zeta$-function (in position space with coordinates $\boldsymbol{x}$ ) as

$$
\boldsymbol{\beta}^{\mathrm{pl}, \boldsymbol{b}}(\boldsymbol{x})=\zeta(\boldsymbol{x}) \boldsymbol{n} \otimes \boldsymbol{b} .
$$

The fundamental equation of plasticity theory,

$$
\boldsymbol{\alpha}^{\boldsymbol{b}}(\boldsymbol{x})=\operatorname{curl} \boldsymbol{\beta}^{\mathrm{pl}, \boldsymbol{b}}(\boldsymbol{x})=\boldsymbol{\rho}(\boldsymbol{x}) \otimes \boldsymbol{b},
$$

yields for the GND vector the relation

$$
\rho(\boldsymbol{x})=\operatorname{curl}(\zeta(\boldsymbol{x}) \boldsymbol{n})=\operatorname{grad} \zeta(\boldsymbol{x}) \times \boldsymbol{n} .
$$


That is, the density vector is the two-dimensional gradient of $\zeta(x)$ tilted by $90^{\circ}$ in clockwise direction in the glide plane. This relation holds in analogous form also for the correlation vector field $\boldsymbol{D}^{(0,1)}(\boldsymbol{r})=\boldsymbol{\rho}^{(0,1)}(\boldsymbol{r} \mid$ disl. at $\mathbf{0})$, which we derive from the gradient of the conditional plastic slip field $\zeta(\boldsymbol{r} \mid$ disl. at $\mathbf{0})$.

For this conditional plastic slip field to be non-trivial, we need to assume that the orientation of the circular dislocations is not equally distributed between circles of positive and negative line sense. The reason is that dislocation loops of opposite line sense cancel each other's contributions to plastic slip when their enclosed circular discs overlap. In the following we assume that all dislocation loops have a positive line sense. The general case may be constructed by superposition of positive and negative density content if the positions of positive and negative loops are uncorrelated.

Given that a positively oriented dislocation loop crosses the origin with unknown direction $\omega, \zeta(\boldsymbol{r} \mid$ disl. at $\mathbf{0})$ is constructed as the probability that the point $\boldsymbol{r}$ lies in the enclosed circular disc of the loop. From Fig. 1 we read that the depicted point at $r$ would be enclosed by all (red) loops (not shown) which cross the origin and lie between the two displayed red loops. The angular range corresponding to the amenable directions of the loops at the origin is $2 \psi$, as depicted by the red dashed circular arc connecting the centres of the two red loops. Because the possible angular range of the tangent vectors at the origin spans $2 \pi$, the probability that the point was 'plastically sheared' is

$$
\zeta(\boldsymbol{r} \mid \text { disl. at } \mathbf{0})=\frac{2 \psi}{2 \pi} .
$$

From the red-blue triangle in Fig. 1 we find the angle $\psi$ to be solely a function of $r$ given by

$$
\psi(r)=\arccos \frac{r}{2 R} .
$$

The conditional plastic slip is consequently

$$
\zeta(\boldsymbol{r} \mid \text { disl. at } \mathbf{0})=\frac{1}{\pi} \arccos \frac{r}{2 R} \text {. }
$$

The graph of this function in dependence on distance $r$ is displayed in Fig. 2. The function approaches the value 0.5 towards the origin, where the covered angular range $\psi$ approaches $\pi$ and consequently each point is covered by about half of the circular discs. The probability to lie within a circular disk first drops linearly close to the origin, with an initial slope of $1 /(2 \pi R)$. For $r \rightarrow 2 R$ the slope, i.e. the derivative of the function, diverges. Because the function $\zeta(\boldsymbol{r} \mid$ disl. at $\mathbf{0})$ is rotationally invariant and decreasing, the planar gradient of it always points to the origin. Therefore, the correlated dislocation vector field $\boldsymbol{\rho}^{(0,1)}(\boldsymbol{r} \mid$ disl. at $\mathbf{0})$ points in positive circumferential direction and its modulus is given by the negative derivative of $\zeta(\boldsymbol{r} \mid$ disl. at $\mathbf{0})$ with respect to $r$, such that

$$
\boldsymbol{D}^{(0,1)}(\boldsymbol{r})=\boldsymbol{\rho}^{(0,1)}(\boldsymbol{r} \mid \text { disl. at } \mathbf{0})=\frac{\rho}{2 \pi R \sqrt{1-r^{2} /\left(4 R^{2}\right)}} \boldsymbol{n} \times \frac{\boldsymbol{r}}{r}=\frac{\rho}{2 \pi R \sqrt{1-r^{2} /\left(4 R^{2}\right)}}(-\sin \varphi, \cos \varphi) .
$$

The vector field consequently "spins around" the origin, as displayed in Fig. 3. The origin is a singular point of the vector field, because the modulus of the vector field remains nonzero when approaching the origin. The magnitude of the vector field diverges at $r=2 R$. Notably, the magnitude when approaching the origin has the limiting value $\rho /(2 \pi R)=$ $q /(2 \pi)$. This recovers the scalar curvature variable $q$ as a correlation quantity. Indeed, the curvature density is an average information on the relative orientation of neighboring segments and thus a local correlation information. 


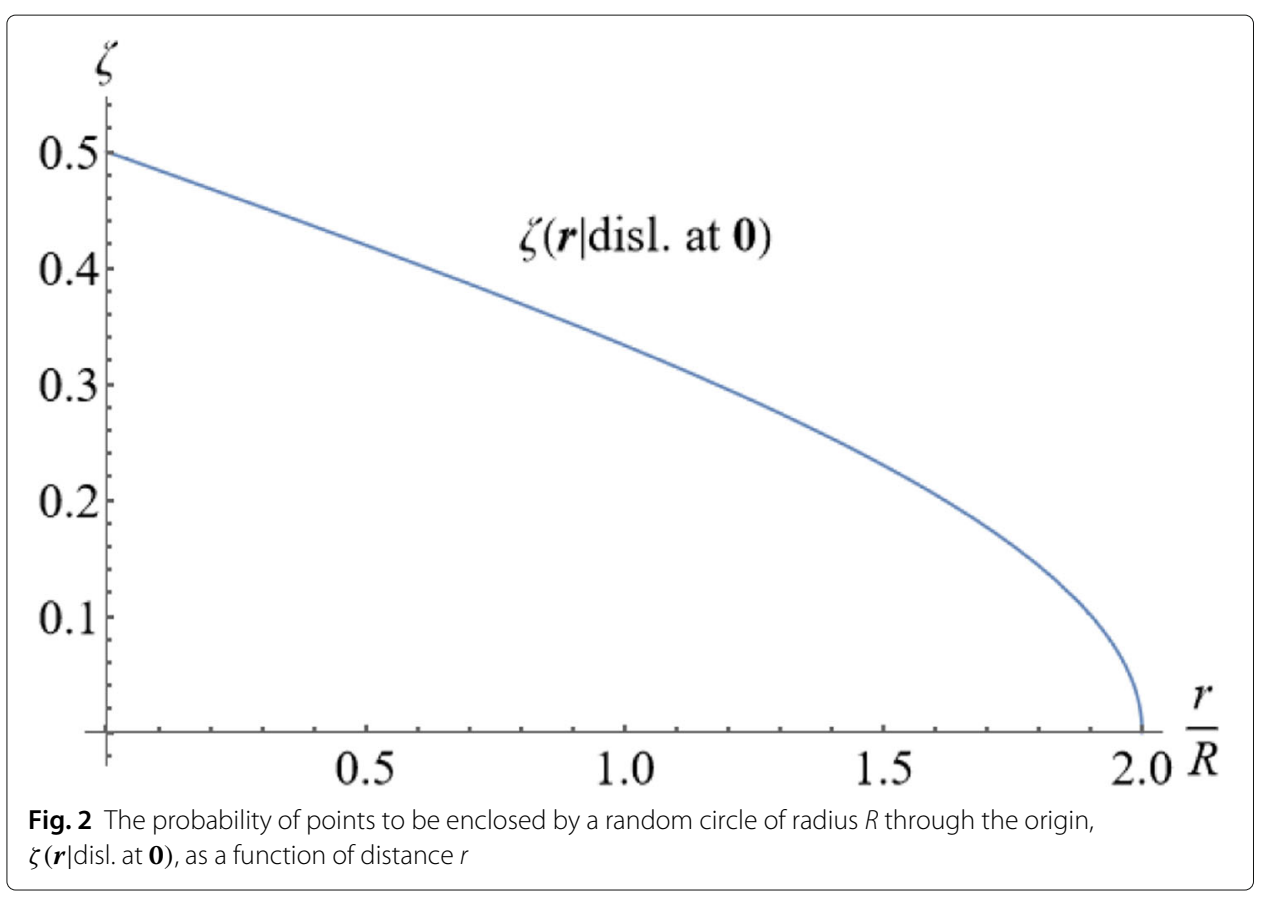

As for the correlation measures of higher order we need to drop the integral approach and have to deal with functions which are no longer rotationally invariant. For the further derivations we therefore switch to a parametric rather than an integral description. We first note that by assuming different circles to be uncorrelated, all non-trivial correlations actually derive as self-correlation of a single, randomly chosen loop. We therefore take recourse to the function $\phi(s, t)$ introduced in Section Correlations in distance space, as obtained for the pairing of a circular loop with itself. Note that in this case the set of distance vectors $H(s, t)$ which arise between points on a circle $c$ of radius $R$ is the circular disc of radius $2 R$ around the origin. Aside from the origin, each point on this disk represents the distance vector between two pairs of points on a circle of radius $R$ for which the distance vector defines a directed secant through the two points. Regarding Fig. 1, the vector $r$ connects a different pair of points on the bold red circle than on the thin one, where however, both circles are considered as representing the same loop in the current consideration.

The two red circles in Fig. 1 provide the two combinations of parameters which map to $r$ as the distance vector, i.e. for positively oriented circles parametrized by $\varsigma \in[0,2 \pi)$ as $\boldsymbol{c}(\varsigma)=\left(m_{x}, m_{y}\right)+R(\cos \varsigma, \sin \varsigma)$, with the (irrelevant) centerpoint $\boldsymbol{m}=\left(m_{x}, m_{y}\right)$, we have (compare red loops in Fig. 1)

$$
H^{-1}(\boldsymbol{r})=\{(\omega-\pi / 2, \omega+\alpha-\pi / 2),(\omega+\alpha+\pi / 2, \omega+\pi / 2)\} .
$$

At either set of parameters the angle between the tangent vectors at the origin and at $r$ is $\pm \alpha$, such that

$$
\phi\left((\varsigma, \tau) \in H^{-1}(\boldsymbol{r})\right)=\frac{\left|\partial_{\zeta} \boldsymbol{c}(\varsigma)\right|\left|\partial_{\tau} \boldsymbol{c}(\tau)\right|}{\left|\partial_{\zeta} \boldsymbol{c}(\varsigma) \times \partial_{\tau} \boldsymbol{c}(\tau)\right|}=\frac{1}{|\sin \alpha|},
$$




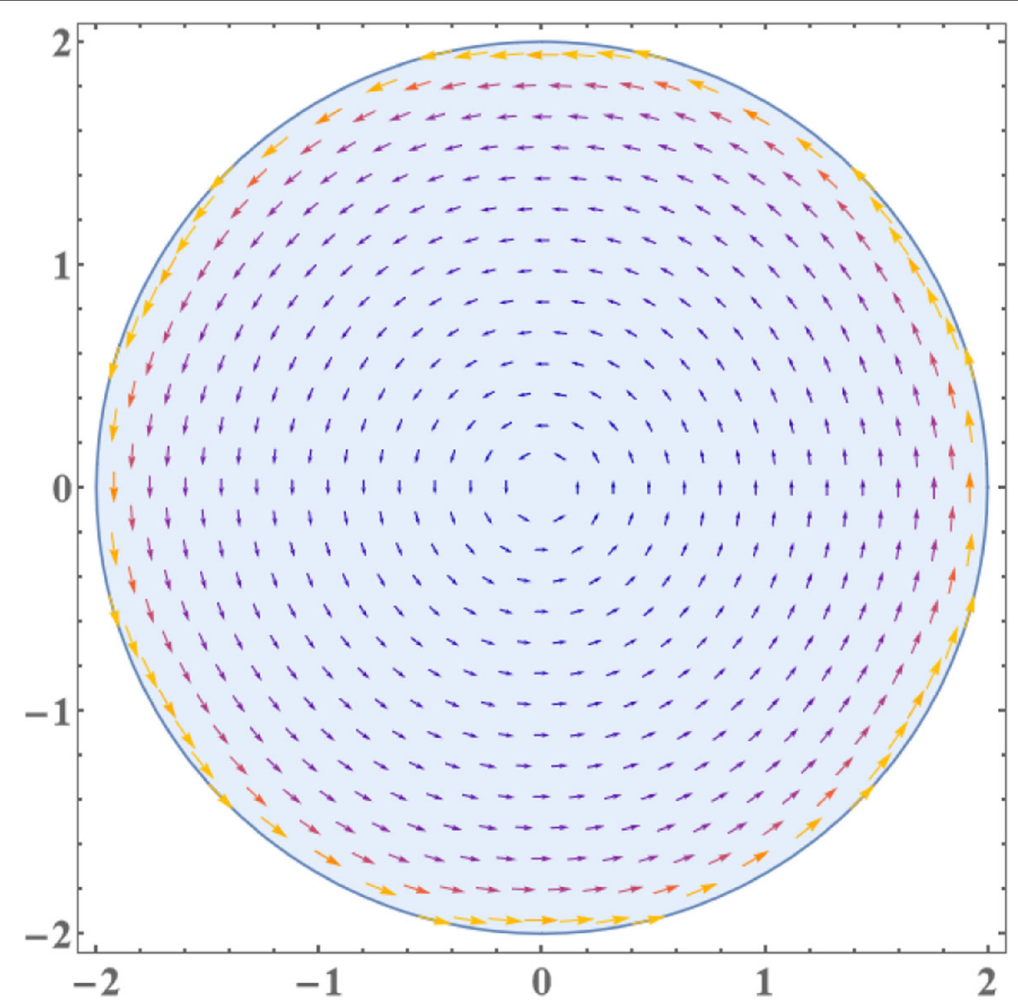

Fig. 3 GND vector field $\boldsymbol{\rho}^{(0,1)}$ as induced by positively oriented circles of radius $R=1$. Length and color of the arrows indicate the magnitude of the vectors, qualitatively. Note that the magnitude diverges at $r=2 R$

and consequently

$$
\phi(\boldsymbol{r})=2 \phi\left((\varsigma, \tau) \in H^{-1}(\boldsymbol{r})\right)=\frac{2}{|\sin \alpha|} .
$$

For the further analysis it is helpful to work with the angle $\beta(r)=\alpha(r) / 2=\arcsin r / 2 R$ (compare Fig. 1 and Eq. (52)). Employing the trigonometric identities

$$
\sin \alpha=\sin 2 \beta=2 \sin \beta \cos \beta \quad \text { and } \quad \cos \beta= \pm \sqrt{1-\sin ^{2} \beta},
$$

and inserting the definition of $\beta$ into Eq. (66) we obtain

$$
\phi(r)=\frac{2 R}{r \sqrt{1-\left(\frac{r}{2 R}\right)^{2}}} .
$$

This function relates to the second summand in Eq. (55) and the pair correlation function in (56) as

$$
\begin{aligned}
D^{(0,0)}(\boldsymbol{r}) & =\frac{\rho}{2 \pi R} \phi(\boldsymbol{r})=\rho_{\mathrm{c}} \phi(\boldsymbol{r})=\frac{q}{2 \pi} \phi(\boldsymbol{r}), \\
d^{(0,0)}(\boldsymbol{r}) & =\frac{\rho_{\mathrm{c}}}{\rho^{2}} \phi(\boldsymbol{r}) .
\end{aligned}
$$

Also $\rho^{(0,1)}$ as derived in Eq. (63) may be recovered from this parametric approach. To this end we define $\boldsymbol{\phi}^{(0,1)}(\boldsymbol{r})$ for a positively oriented loop. For this we need to add together the two unit tangent vectors through $r$. Realizing the positive line sense of the two red circles, the tangent vector to the bold-lined and thin-lined red circles at position $r$ are 


$$
\boldsymbol{l}_{\mathrm{bold}, \boldsymbol{r}}(\boldsymbol{r})=(\cos (\varphi+\beta), \sin (\varphi+\beta)) \text { and } \boldsymbol{l}_{\mathrm{thin}, \boldsymbol{r}}(\boldsymbol{r})=(\cos (\varphi+\pi-\beta), \sin (\varphi+\pi-\beta)) \text {, }
$$

respectively. The angles $\varphi$ and $\beta$ are functions of $\boldsymbol{r}$ (compare Fig. 1), which is not included in the notation for brevity. Note, moreover, that we regard the full intersecting circles as being determined by, i.e., as functions of, the point $\boldsymbol{r}$. The tangent vectors $\boldsymbol{l}$ to these circles are thus on the one hand functions of $\boldsymbol{r}$, as indicated by their dependence on $\varphi$ and $\beta$. But the tangents can in principle be evaluated at any point on the circles, which is why the position, that is again $r$, additionally appears as subscript. This, in the current case seemingly redundant, notation will be needed when evaluating the tangents of these circles at the origin.

The sum of the tangent vectors of the red circles with positive line sense in point $\boldsymbol{r}$ yields a non-normalized vector $\boldsymbol{s}$ which is tangent to the blue circle,

$$
\boldsymbol{s}=\boldsymbol{l}_{\mathrm{bold}, \boldsymbol{r}}+\boldsymbol{l}_{\mathrm{thin}, \boldsymbol{r}}=2 \sin \beta(-\sin \varphi, \cos \varphi) .
$$

Consequently we have

$$
\phi^{(0,1)}(\boldsymbol{r})=\frac{2 \sin \beta}{|\sin \alpha|}(-\sin \varphi, \cos \varphi) .
$$

Employing the first trigonometric identity in Eq. (67) we obtain

$$
\phi^{(0,1)}(\boldsymbol{r})=\frac{1}{|\cos \beta|}(-\sin \varphi, \cos \varphi)=\frac{1}{\sqrt{1-\left(\frac{r}{2 R}\right)^{2}}}(-\sin \varphi, \cos \varphi) .
$$

The relation to $\boldsymbol{D}^{(0,1)}$ is given, analogously to the last equality in Eq. (69)

$$
\boldsymbol{D}^{(0,1)}(\boldsymbol{r})=\frac{q}{2 \pi} \phi^{(0,1)}(\boldsymbol{r}) \text {. }
$$

Indeed, the non-normalized correlation tensors may all be obtained as

$$
\boldsymbol{D}^{\left(n, n^{\prime}\right)}(\boldsymbol{r})=\frac{q}{2 \pi} \boldsymbol{\phi}^{\left(n, n^{\prime}\right)}(\boldsymbol{r}) \text {. }
$$

We provide two further examples of correlation tensors to illustrate the distinction of the vector-vector correlation $\boldsymbol{D}^{(1,1)}$ and the scalar to second order tensor correlation $\boldsymbol{D}^{(0,2)}$.

In order to calculate $\boldsymbol{\phi}^{(1,1)}$ we additionally need the tangent vectors at the origin to the bold and thin red circles in Fig. 1, which are

$$
\boldsymbol{l}_{\text {bold }, \mathbf{0}}(\boldsymbol{r})=(\cos (\varphi-\beta), \sin (\varphi-\beta)) \text { and } \boldsymbol{l}_{\text {thin, } \mathbf{0}}(\boldsymbol{r})=(\cos (\varphi+\pi+\beta), \sin (\varphi+\pi+\beta)) .
$$

With these we find

$$
\boldsymbol{D}^{(1,1)}(\boldsymbol{r})=\frac{q}{2 \pi}\left(\boldsymbol{l}_{\mathrm{bold}, \mathbf{0}}(\boldsymbol{r}) \otimes \boldsymbol{l}_{\mathrm{bold}, \boldsymbol{r}}(\boldsymbol{r})+\boldsymbol{l}_{\mathrm{thin}, \mathbf{0}}(\boldsymbol{r}) \otimes \boldsymbol{l}_{\mathrm{thin}, \boldsymbol{r}}(\boldsymbol{r})\right) \phi(\boldsymbol{r}) .
$$

The orientation of the circles does not matter here, because it would invert each time both vectors in the tensor products. Note that the subscript at the tangents indicate that $\boldsymbol{D}^{(1,1)}(\boldsymbol{r})$ is not a tensor of second order but a two-point tensor, mapping (unit) vectors $\boldsymbol{e}$ at the origin to a vector at $\boldsymbol{r}$ (or vice versa), i.e. it assigns to such vectors a correlated GND vector field. The vector field $\boldsymbol{e} \cdot \boldsymbol{D}^{(1,1)}(\boldsymbol{r})$ thus indicates the expected GND vector at $\boldsymbol{r}$, knowing that there is a dislocation with tangent component in unit direction $\boldsymbol{e}$ at the origin. Figure 4 shows the vector fields obtained for $\boldsymbol{e}=(1,0)$ (a) and $\boldsymbol{e}=1 / \sqrt{2}(1,1)$ (b). As may be seen, the expected vector fields are related to each other by a rigid rotation, 


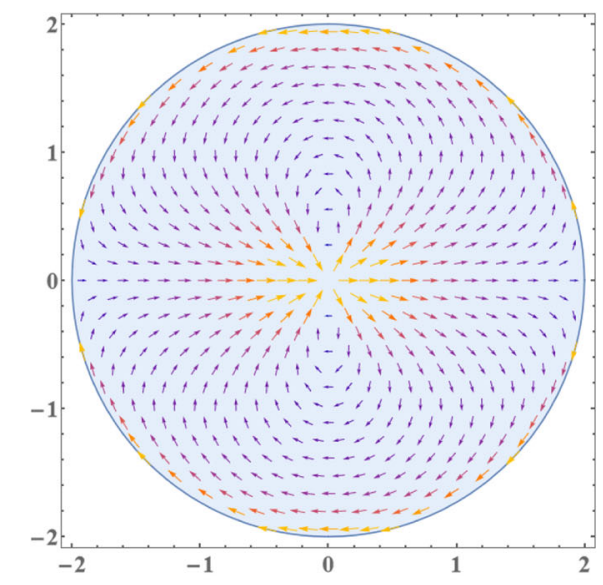

(a)

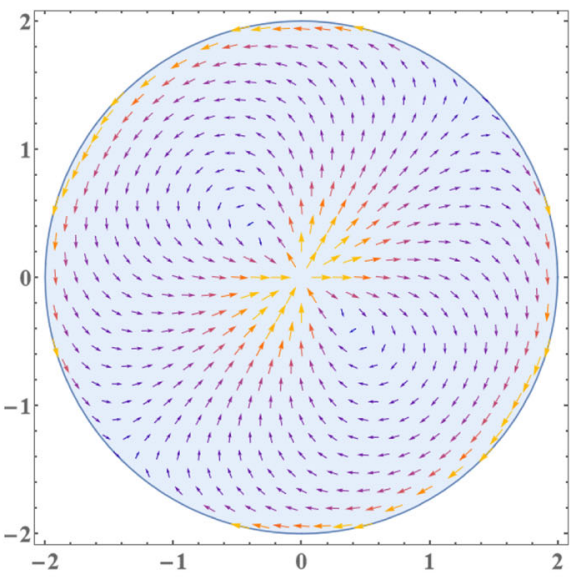

(b)

Fig. 4 Vector fields $(1,0) \cdot \boldsymbol{D}^{(1,1)}(\boldsymbol{r})(\mathbf{a})$ and $1 / \sqrt{2}(1,1) \cdot \boldsymbol{D}^{(1,1)}(\boldsymbol{r})(\mathbf{b})$ in homogeneous distributions of loops with radius $R=1$. Length and color of the arrows indicate the magnitude of the vectors, qualitatively

which is the rotation between the vectors at the origin. In either case there is a singular expectation to find similar GND directions close to the origin, most notably along the direction of $\boldsymbol{e}$. The vector field 'turns back' at $2 R$ distance in direction perpendicular to $\boldsymbol{e}$. Again we note that it is the central part which we believe is generic whereas what happens close to $r=2 R$ is an artifact of the fixed radius.

While we think it is helpful to consider the content of $\boldsymbol{D}^{(1,1)}(\boldsymbol{r})$ in the above vector field interpretation, we also provide contour plots of the absolute values of the coefficient functions $D_{x x}^{(1,1)}(\boldsymbol{r}), D_{x y}^{(1,1)}(\boldsymbol{r})=D_{y x}^{(1,1)}(\boldsymbol{r})$, and $D_{y y}^{(1,1)}(\boldsymbol{r})$ in Fig. 5 in order to connect to the work of Anderson and El-Azab (2021). In Fig. 5 the functions are only displayed for $r<R$ and not in the covered space of distance up to $2 R$. This avoids the visual dominance of the artificial singularities from the fixed radius. The functions qualitatively resemble -as they should- the screw-screw, $\left|D_{x x}^{(1,1)}(\boldsymbol{r})\right|$, screw-edge, $\left|D_{x y}^{(1,1)}(\boldsymbol{r})\right|$, and edge-edge correlations, $\left|D_{y y}^{(1,1)}(\boldsymbol{r})\right|$, as obtained by Anderson and El-Azab (2021) when suitably normalized, cf. Fig. $8 \mathrm{~b}$ in the named paper.

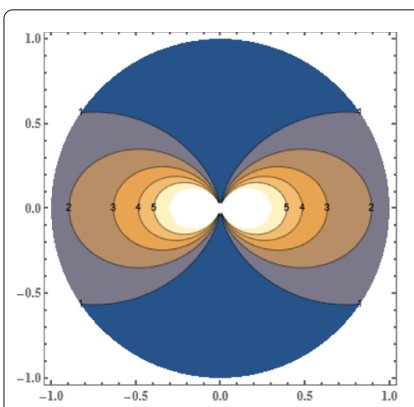

(a) $\left|D_{x x}^{(1,1)}\right|$ in $\frac{q}{2 \pi}$

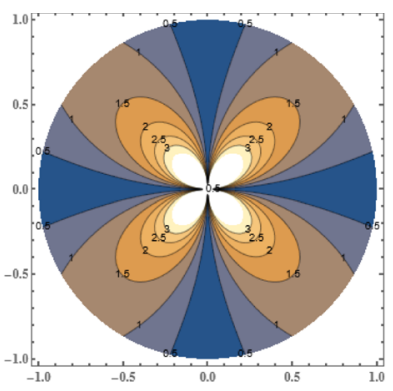

(b) $\left|D_{x y}^{(1,1)}\right|$ in $\frac{q}{2 \pi}$

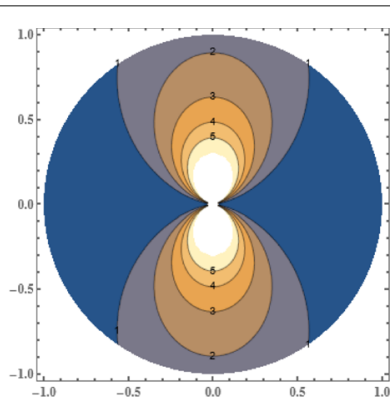

(c) $\left|D_{y y}^{(1,1)}\right|$ in $\frac{q}{2 \pi}$

Fig. 5 Contour plots of the absolute values of the coefficient functions $D_{x x}^{(1,1)}(\boldsymbol{r})(\mathbf{a}), D_{x y}^{(1,1)}(\boldsymbol{r})=D_{y x}^{(1,1)}(\boldsymbol{r})(\mathbf{b})$, and $D_{y y}^{(1,1)}(\boldsymbol{r})(\mathbf{c})$ in a homogeneous distribution of loops with radius $R=1$. Contours are labeled by function value. Note that as opposed to the other plots in this section, these plots are restricted to distances $r<1$ from the origin 
We now regard the expected second order alignment tensor $\boldsymbol{D}^{(0,2)}(\boldsymbol{r})$ around a generic point in a homogeneous distribution of circles. This is given by

$$
\boldsymbol{D}^{(0,2)}(\boldsymbol{r})=\frac{q}{2 \pi}\left(\boldsymbol{l}_{\mathrm{bold}, \boldsymbol{r}}(\boldsymbol{r}) \otimes \boldsymbol{l}_{\mathrm{bold}, \boldsymbol{r}}(\boldsymbol{r})+\boldsymbol{l}_{\mathrm{thin}, \boldsymbol{r}}(\boldsymbol{r}) \otimes \boldsymbol{l}_{\mathrm{thin}, \boldsymbol{r}}(\boldsymbol{r})\right) \phi(\boldsymbol{r}) .
$$

Note that as opposed to the case of $\boldsymbol{D}^{(1,1)}(\boldsymbol{r})$ in Eq. (78), the tangent vectors are now evaluated each time at position $\boldsymbol{r}$, as indicated by the subscripts. The result is consequently a symmetric second order tensor at $\boldsymbol{r}$, subject to the condition that there is a dislocation of unknown tangent direction at the origin. One may rationalize from the symmetry of the regarded distribution or infer from the definition of $\boldsymbol{D}^{(0,2)}(\boldsymbol{r})$ by the sum of two exterior products of unit vectors, that the eigenvectors of the tensor at a point $\boldsymbol{r}$ are the unit vectors in radial direction $\boldsymbol{e}_{r}(\boldsymbol{r})=(\cos \varphi, \sin \varphi)$ and circumferential direction $\boldsymbol{e}_{\varphi}(\boldsymbol{r})=(-\sin \varphi, \cos \varphi)$, where we note that $\boldsymbol{e}_{r}(\boldsymbol{r}) \|\left(\boldsymbol{l}_{\mathrm{bold}, \boldsymbol{r}}(\boldsymbol{r})-\boldsymbol{l}_{\mathrm{thin}, \boldsymbol{r}}(\boldsymbol{r})\right)$ and $\boldsymbol{e}_{\varphi}(\boldsymbol{r}) \|\left(\boldsymbol{l}_{\mathrm{bold}, \boldsymbol{r}}(\boldsymbol{r})+\boldsymbol{l}_{\mathrm{thin}, \boldsymbol{r}}(\boldsymbol{r})\right)$. With these eigenvectors the tensor is expressed in diagonal form as

$$
\boldsymbol{D}^{(0,2)}(\boldsymbol{r})=\frac{q}{2 \pi}\left[\sqrt{\left(\frac{4 R^{2}-r^{2}}{r^{2}}\right)} \boldsymbol{e}_{r}(\boldsymbol{r}) \otimes \boldsymbol{e}_{r}(\boldsymbol{r})+\sqrt{\left(\frac{r^{2}}{4 R^{2}-r^{2}}\right)} \boldsymbol{e}_{\varphi}(\boldsymbol{r}) \otimes \boldsymbol{e}_{\varphi}(\boldsymbol{r})\right] .
$$

This tensor field is visualized in Fig. 6 by overlaying two fields of double arrows in eigenvector direction with their length scaled by the according eigenvalue. Around the origin dislocations are expected to be strongly radially aligned (diverging at the origin), while spherical tensors (equal eigenvalues, that is no alignment) are found for $r=\sqrt{2} R$, and diverging circumferential alignment is found when approaching the diameter, i.e. for $r \rightarrow 2 R$

As final result in this subsection we provide the universal formula for the correlation tensors in isotropic circle distributions, which we shall generalize to non-isotropic distributions in the next subsection. The correlation tensors are given by

$$
\boldsymbol{D}^{\left(n, n^{\prime}\right)}(\boldsymbol{r})=\frac{q}{2 \pi}\left(\boldsymbol{l}_{\mathrm{bold}, \mathbf{0}}^{\otimes n}(\boldsymbol{r}) \otimes \boldsymbol{l}_{\mathrm{bold}, \boldsymbol{r}}^{\otimes n^{\prime}}(\boldsymbol{r})+\boldsymbol{l}_{\mathrm{thin}, \mathbf{0}}^{\otimes n}(\boldsymbol{r}) \otimes \boldsymbol{l}_{\mathrm{thin}, \boldsymbol{r}}^{\otimes n^{\prime}}(\boldsymbol{r})\right) \phi(\boldsymbol{r}),
$$

if only circles of positive line sense are considered. If circles of positive and negative density are considered with equal probability we obtain

$$
\boldsymbol{D}^{\left(n, n^{\prime}\right)}(\boldsymbol{r})=\frac{q}{2 \pi} \frac{1+(-1)^{n+n^{\prime}}}{2}\left(\boldsymbol{l}_{\mathrm{bold}, \mathbf{0}}^{\otimes n}(\boldsymbol{r}) \otimes \boldsymbol{l}_{\mathrm{bold}, \boldsymbol{r}}^{\otimes n^{\prime}}(\boldsymbol{r})+\boldsymbol{l}_{\mathrm{thin}, \mathbf{0}}^{\otimes n}(\boldsymbol{r}) \otimes \boldsymbol{l}_{\mathrm{thin}, \boldsymbol{r}}^{\otimes n^{\prime}}(\boldsymbol{r})\right) \phi(\boldsymbol{r}),
$$

such that if $n+n^{\prime}$ is even, this reduces to Eq. (81), while all other tensors vanish. Formulas for more general distributions between positive and negative loops may be obtained by superposition.

\section{Self-correlations in polarized and non-isotropic distributions}

So far we concentrated on a for dislocations completely unrealistic and very specific distribution of homogeneously distributed circles. However, when at a point the (planar) CDD variables $\rho, q$, and $\rho=0$ are given, it seems natural to assume that the immediate surrounding of that point is, with regard to self-correlations, on average similar to the surrounding of the origin in a distribution of circles with radius $R=\frac{\rho}{|q|}$. If one accepts this premise, this idea may be easily generalized to other known CDD variables $q, \rho=\rho^{(0)}, \boldsymbol{\rho}^{(1)}, \boldsymbol{\rho}^{(2)}, \ldots, \boldsymbol{\rho}^{(n)}$ at a point, as long as only isotropic curvature information 


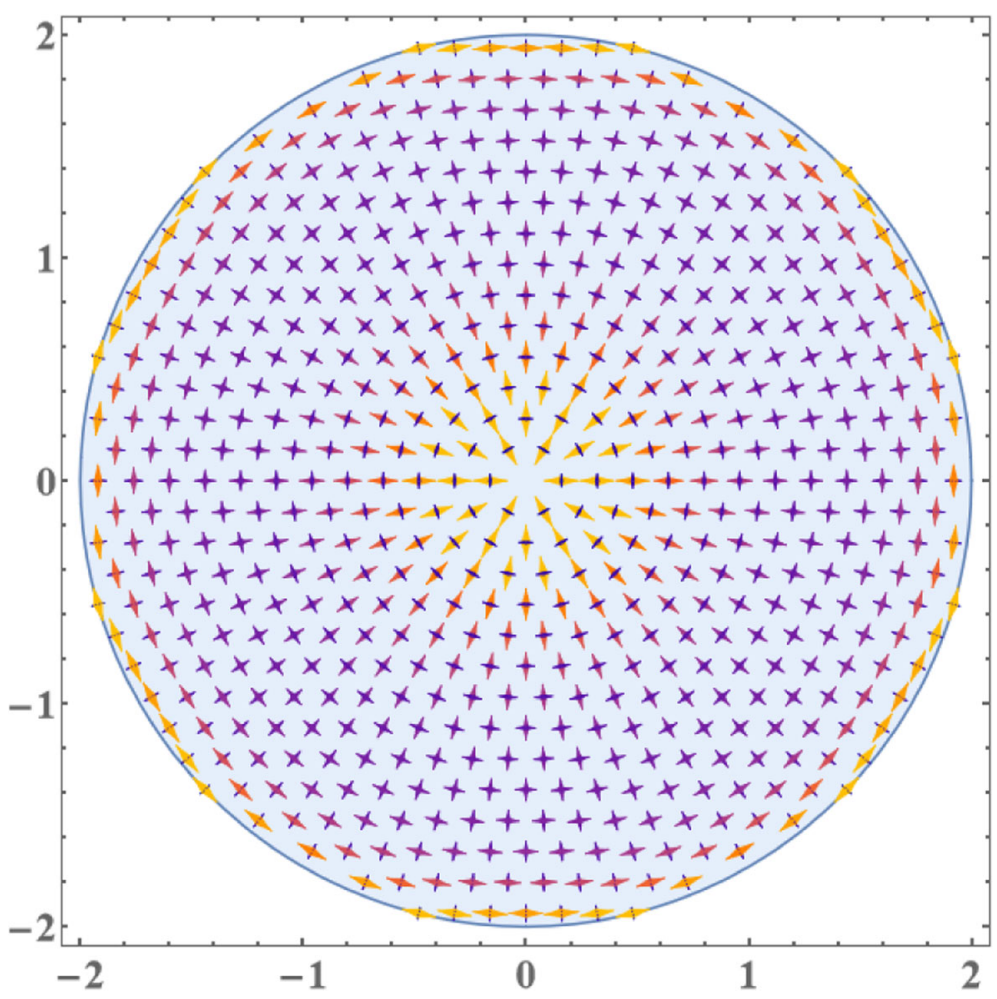

Fig. 6 The correlation field of the alignment tensor $\boldsymbol{D}^{(0,2)}(\boldsymbol{r})$ in a homogeneous distribution of loops with radius $R=1$ visualized by two fields of double arrows in eigenvector directions. Length and color of the arrows indicate the magnitude of the vectors, qualitatively

is considered. The more general case when also higher order curvature tensors are given will be treated elsewhere.

The idea is, that if we know the probability $\rho(\omega)$ to find a circle with a line direction given through the angle $\omega$ at the origin, this determines the probability to find segments with a given connecting vector and thus the self-correlations around the origin. Monavari et al. $(2014,2016)$ have shown that the maximum information entropy principle may be used to construct a directional dependent density $\rho(\omega)$ from a given number of CDD density variables. The function $\rho(\omega)$ is designed to maximize the information entropy functional

$$
H[\rho]=-\int_{0}^{2 \pi} \frac{\rho(\omega)}{\rho} \ln \frac{\rho(\omega)}{\rho} \mathrm{d} \omega,
$$

subject to the side conditions

$$
\int_{0}^{2 \pi} \rho(\omega) \boldsymbol{l}^{\otimes k}(\omega) \mathrm{d} \omega=\boldsymbol{\rho}^{(k)} \text { for } k=0, \ldots, n .
$$

Entropy maximization is commonly considered to yield the closure which involves the least additional information aside from the side conditions. For the zeroth order closure $(n=0)$, that is for just $\rho$ (and $q$ ) given, maximum entropy closure yields $\rho(\omega)=\rho / 2 \pi$. If in addition $\rho(n=1)$ is given, the maximum entropy distribution is a von Mises-Fisher distribution of the form

$$
\rho(\omega)=\frac{\rho}{2 \pi \mathrm{I}_{0}(\Lambda)} \exp \left(\Lambda \cos \left(\omega-\omega_{\rho}\right)\right)
$$


where $\omega_{\rho}$ denotes the angle of the GND direction and $\mathrm{I}_{0}$ denotes the modified Bessel function of order 0 . The coefficient $\Lambda$ (Lagrange multiplier) is obtained from solving

$$
\frac{\mathrm{I}_{1}(\Lambda)}{\mathrm{I}_{0}(\Lambda)}=\frac{|\rho|}{\rho},
$$

where $I_{1}$ is the next higher order modified Bessel function. An approximate solution for Eq. (86) has been given by Monavari et al. (2014). If dislocation density tensors of higher order are given, the maximum entropy distribution has to be obtained from solving a strongly non-linear system of equations for the Lagrange multipliers of the side conditions. This will usually require employing numerical methods, for what we use a standard Newton-Raphson algorithm.

Assuming a suitable closure for $\rho(\omega)$ is given, which fulfills the side conditions in Eq. (85), the correlation tensors around the given point may be estimated by assuming that the tangents to the circles through the origin are distributed in orientation space by $\rho(\omega)$. Because the radius $R=\frac{\rho}{|q|}$ is independent of the angle we can employ the same reasoning as above to determine the possible orientations (at the origin) of circles connecting a point $\boldsymbol{r}$ with the origin. Note that we employ the sign of $q$ as to determine the sense of orientation, where positive curvature corresponds to a positive line sense. We accordingly only generalize Eq. (81), while a generalization of Eq. (82) would require considering CDD variables for positively and negatively oriented loops separately. We denote the angles corresponding to the line directions at the origin, $\boldsymbol{l}_{\text {bold }, \mathbf{0}}$ and $\boldsymbol{l}_{\text {thin, } \mathbf{0}}$ with $\omega_{\text {bold, } \mathbf{0}}$ and $\omega_{\text {thin, }, 0}$, respectively. With this notation we find

$$
\boldsymbol{D}^{\left(n, n^{\prime}\right)}(\boldsymbol{r})=\left(\rho\left(\omega_{\mathrm{bold}, \mathbf{0}}\right) \boldsymbol{l}_{\mathrm{bold}, \mathbf{0}}^{\otimes n}(\boldsymbol{r}) \otimes \boldsymbol{l}_{\mathrm{bold}, \boldsymbol{r}}^{\otimes n^{\prime}}(\boldsymbol{r})+\rho\left(\omega_{\mathrm{thin}, \mathbf{0}}\right) \boldsymbol{l}_{\mathrm{thin}, \mathbf{0}}^{\otimes n}(\boldsymbol{r}) \otimes \boldsymbol{l}_{\mathrm{thin}, \boldsymbol{r}}^{\otimes n^{\prime}}(\boldsymbol{r})\right) \frac{\phi(\boldsymbol{r})}{R},
$$

where we remind that the line directions, the angles, and the function $\phi(r)$ all depend on the radius $R=\frac{\rho}{|q|}$, which is not explicitly indicated in the notation.

We provide two examples to illustrate the effect of polarization, $\boldsymbol{\rho}^{(1)} \neq 0$ and alignment, $\boldsymbol{\rho}^{(2)} \neq 1 / 2 \rho^{(0)} \mathbf{I}$, where $\mathbf{I}$ denotes the two-dimensional unit matrix. We begin with a polarization in $x$-direction, where $\boldsymbol{\rho}^{(1)}=0.25 \rho(1,0)$. The polarization indicates that there is a surplus of circles whose tangents at the origin have a positive $x$ component as compared to those with negative $x$ component. That is, we expect more circles above the origin than below it. For this case we obtained the orientation distributions by a NewtonRaphson algorithm with the aid of Mathematica ${ }^{\circledR}$. The results are displayed in Fig. 7. The dominance of circles in the upper half clearly displays in vertical asymmetries in the total dislocation density (a), the GND density associated with the direction $(1,0)$ at the origin (b), and the correlated alignment tensor field $\boldsymbol{D}^{(0,2)}$ (c).

As a second example we regard an unpolarized (i.e., $\rho^{(1)}=0$ ) distribution with a preferred alignment in $\boldsymbol{e}_{x}=(1,0)$-direction such that

$$
\rho^{(2)}=0.625 \rho^{(0)} \boldsymbol{e}_{x} \otimes \boldsymbol{e}_{x}+0.375 \rho^{(0)} \boldsymbol{e}_{y} \otimes \boldsymbol{e}_{y} .
$$

In this case there are altogether more circles with horizontal tangent components at the origin than there are with vertical tangent component, irrespective of the line senses. We therefore expect higher correlations in horizontal direction than vertically. The according correlation fields are displayed in Fig. 8. The alignment clearly displays in the total dislocation density (a) and in the second order alignment tensor (c) by breaking the rotational 


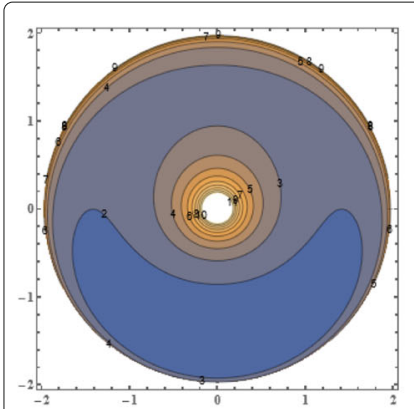

(a)

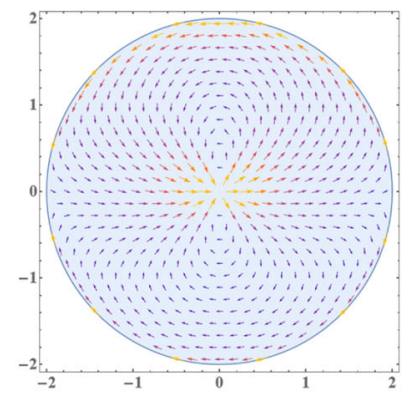

(b)

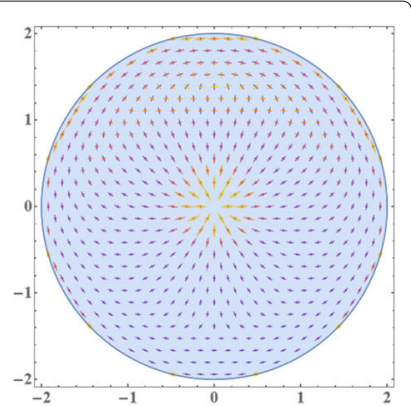

(c)

Fig. 7 The correlation fields $D^{(0,0)}(\boldsymbol{r})$ in $\frac{q}{2 \pi}(\mathbf{a}),(1,0) \cdot \boldsymbol{D}^{(1,1)}(\boldsymbol{r})(\mathbf{b})$, and $\boldsymbol{D}^{(0,2)}(\boldsymbol{r})(\mathbf{c})$ in homogeneous distributions of loops with polarization in horizontal direction as described in the text. Contours are labeled by function value in (a). Length and color of the arrows indicate the magnitude of the vectors, qualitatively, in (b) and (c)

symmetry. As for the GND field induced by the direction $\boldsymbol{e}=1 / \sqrt{2}(1,1)$ at the origin (b), a careful look reveals that the vector field does not possess the mirror symmetry around the main diagonal which was given in the isotropic case, cf. Fig. 4b. Around the center, the vector field appears to be 'turned' towards the dominating horizontal direction, when compared to the isotropic case where the vector field clearly follows the diagonal.

\section{Correlations in homogeneous systems with a distribution of radii}

In the above analysis we restricted ourselves to the very artificial case of a distributions of circles with the same radius. Complementary to the generalization to polarized and non-isotropic distributions, we shall now briefly discuss how to obtain self-correlations when we allow for a distribution $f(R)$ of radii. We assume a total density $\rho$ and the average curvature $q$ are given. The center points are again assumed to follow a Poisson point process with density $\rho_{\mathrm{c}}=q / 2 \pi$. Then the distribution of radii fulfills

$$
\int_{-\infty}^{\infty} f(R) d R=\rho_{\mathrm{c}}
$$

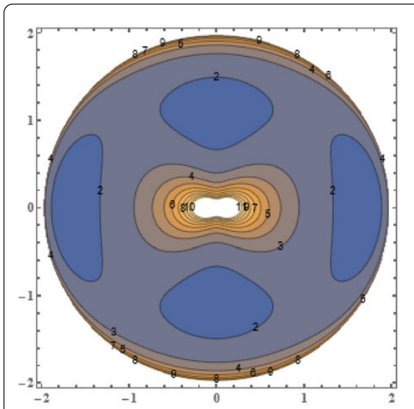

(a)

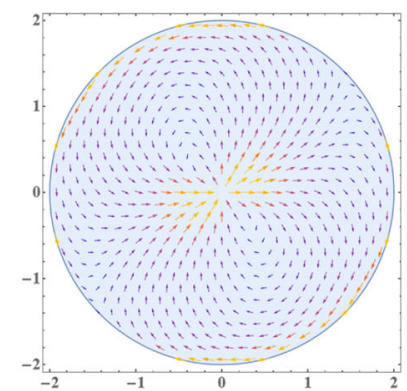

(b)

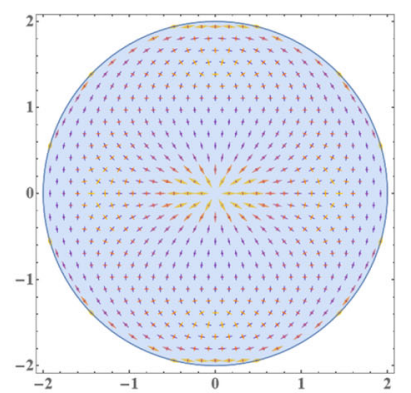

(c)

Fig. 8 The correlation fields $D^{(0,0)}(\boldsymbol{r})$ in $\frac{9}{2 \pi}(\mathbf{a}), 1 / \sqrt{2}(1,1) \cdot \boldsymbol{D}^{(1,1)}(\boldsymbol{r})(\mathbf{b})$, and $\boldsymbol{D}^{(0,2)}(\boldsymbol{r})$ in homogeneous distributions of loops with preferred alignment in horizontal direction as described in the text. Contours are labeled by function value in (a). Length and color of the arrows indicate the magnitude of the vectors, qualitatively, in (b) and (c) 
and the dislocation density results as

$$
\int_{-\infty}^{\infty} 2 \pi R f(R) d R=\rho
$$

It is convenient to reformulate this with a probability density $p(R)=f(R) / \rho_{\mathrm{c}}$ for which

$$
\begin{aligned}
\int_{-\infty}^{\infty} p(R) d R & =1 \\
\int_{-\infty}^{\infty} R p(R) d R & =\frac{\rho}{q}=: \bar{R}
\end{aligned}
$$

where $\bar{R}$ denotes the average radius.

We now note that the unscaled correlation tensors $\boldsymbol{D}^{\left(n, n^{\prime}\right)}$ and the tensor function $\boldsymbol{\phi}^{\left(n, n^{\prime}\right)}$, which we considered solely as functions of the position vector $\boldsymbol{r}$ before, are also functions of the loop radius $R$. The overall correlation function as a function of space is accordingly obtained by integrating the correlation functions as functions of $R$ against the radius distribution, that is

$$
\boldsymbol{D}^{\left(n, n^{\prime}\right)}(\boldsymbol{r})=\int_{-\infty}^{\infty} \boldsymbol{\phi}^{\left(n, n^{\prime}\right)}(\boldsymbol{r}, R) f(R) \mathrm{d} R=\frac{q}{2 \pi} \int_{-\infty}^{\infty} \boldsymbol{\phi}^{\left(n, n^{\prime}\right)}(\boldsymbol{r}, R) p(R) \mathrm{d} R .
$$

Note that we recover the case of a single radius, which is now called $\bar{R}$, if the density is a Delta-distribution $p(R)=\delta(R-\bar{R})$.

In order to illustrate the consequence of considering distributions of radii, we assume the radii to follow a log-normal distribution with parameters $\mu$ and $\sigma^{2}$ for positive radii, $R>0$ and zero for $R<0$. That is, we assume the probability density to be of the form

$$
p(R)=\frac{1}{\sqrt{2 \pi} \sigma R} \exp \left(-\frac{(\ln R-\mu)^{2}}{2 \sigma^{2}}\right) \Theta(R),
$$

where $\Theta(R)$ denotes the Heaviside step function, which is zero for negative and 1 for positive arguments. The average radius, i.e. the expectation value of the log-normal distribution is $\bar{R}=\exp \left(\mu+\frac{\sigma^{2}}{2}\right)$. In the sequel we set $\bar{R}=1$ such that the admissible parameters fulfill $\sigma^{2}=-2 \mu$.

In Fig. 9a we display three variants of such log-normal density distributions and the fourth one formally arises by letting $\sigma$ and $\mu$ simultaneously go to zero, which recovers the Dirac delta function $p(R)=\delta(R-1)$. Besides the Dirac delta, we chose the parameters such that the distributions range from a narrow $\left(\mu=-1 / 512, \sigma^{2}=1 / 256\right)$ over a moderately narrow $\left(\mu=-1 / 32, \sigma^{2}=1 / 16\right)$ to a wide $\left(\mu=-1 / 2, \sigma^{2}=1\right)$ distribution of radii. In Fig. $9 \mathrm{~b}$ and c we display the according correlation functions $d^{(0,0)}(r)$ as obtained from Eq. (93) via numerical integration with Mathematica ${ }^{\circledR}$. Especially the double logarithmic plot in Fig. 9c illustrates how the $1 / r$ dependence prevails for distances $r \lesssim 1 / 2 \bar{R}$ for all distributions.

As a further example for the consequences of radii distributions on the correlation tensors we provide plots for the modulus of the correlation vector field $\boldsymbol{d}^{(0,1)}$ in Fig. 9d. Note that the direction of the correlation vector fields, as given in Eq. (63), are independent of the radius $R$ and that the moduli are always just a function of distance $r$. Therefore, the correlation vector field obtained from integration over the radii likewise shares the same (circumferential) direction at each point and has a direction independent modulus. The distributions of the moduli of $\boldsymbol{d}^{(0,1)}$ shown in Fig. $9 \mathrm{~d}$ were likewise obtained via numerical integration with Mathematica ${ }^{\circledR}$. As expected, we see that the divergence at $2 R$ disappears when considering distributions of radii. Moreover, the limiting value of the modulus at 


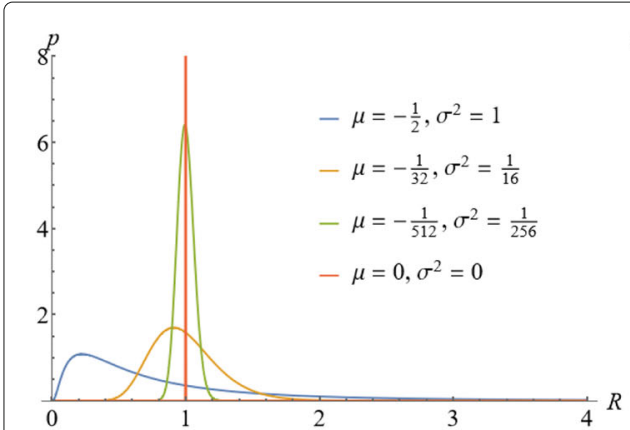

(a)

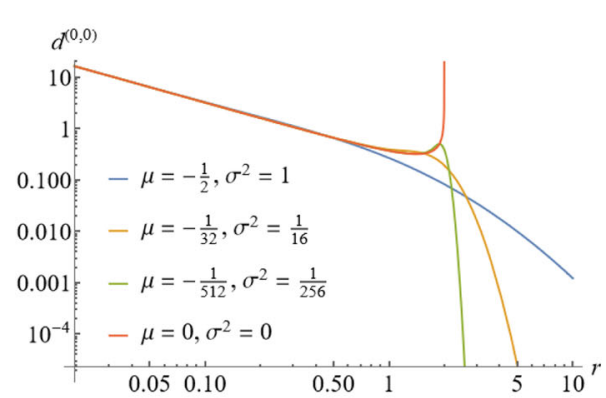

(c)

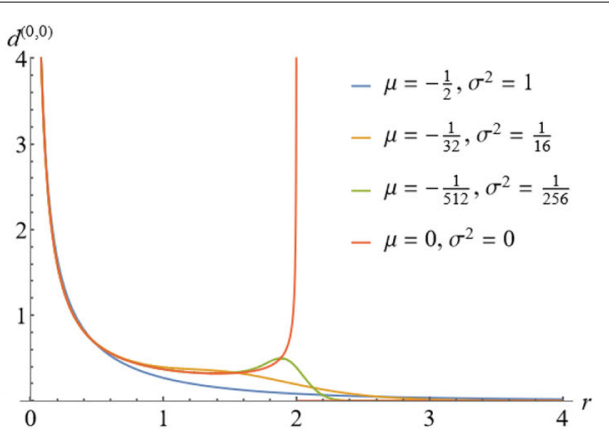

(b)

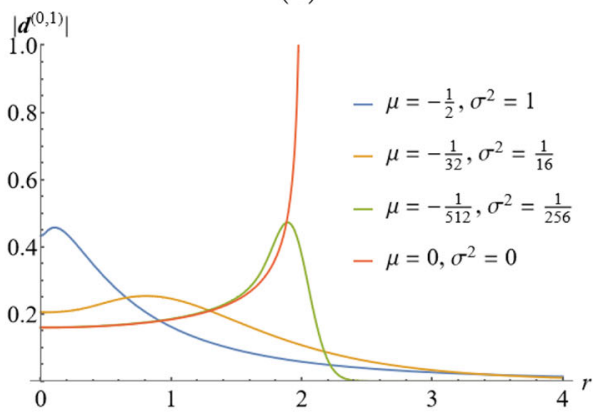

(d)

Fig. 9 Exemplary log-normal probability distributions $p(R)$ with $\bar{R}=1$ displayed for positive radii (a). The scalar correlation function $d^{(0,0)}$ as a function of $r$ with linear $(\mathbf{b})$ and double logarithmic (c) scales, as obtained from Eq. (93) with the distribution functions shown in (a). Modulus of the correlation vector field $\boldsymbol{d}^{(0,1)}$ in dependence on $r(\mathbf{d})$, as obtained from Eq. (93) with the distribution functions shown in (a)

the center increases with the width of the log-normal distributions. This occurs because the log-normal distributions have their maxima for radii smaller than the average radius $\bar{R}$, and thus for higher curvatures. Note that the curvature determines the limiting value in the single radius case.

\section{Correlation tensor evaluation from discrete dislocation data}

The basis for evaluating correlation tensors from discrete dislocation data is Eq. (22), which is based on evaluating single-point quantities for pairs of subvolumes. We therefore begin this section with recalling how to determine the single-point density and curvature tensors from discrete dislocation results based on straight segments connected at nodes, as used in many DDD codes. For the derivation of these definitions from the one for continuous curves in Section Dislocation density and correlation tensors we refer to Weger et al. (2021). For a given volume $V$ we denote with $M(\boldsymbol{b})$ the total number of straight dislocation segments $\boldsymbol{c}_{m}^{\boldsymbol{b}}, m=1, \ldots M(\boldsymbol{b})$ with Burgers vector $\boldsymbol{b}$ contained in the volume. Note that for a subvolume of a simulation box this presumes that segments which intersect the boundary of $V$ have been dissected accordingly. Let moreover $K(\boldsymbol{b})$ be the number of nodes in $V$ at which segments of a Burgers vector $\boldsymbol{b}$ meet. ${ }^{5}$ Let $L_{m}^{\boldsymbol{b}}$ be the length and $\boldsymbol{l}_{m}^{\boldsymbol{b}}$ be the unit tangent direction of segment $\boldsymbol{c}_{m}^{\boldsymbol{b}}$. For a node $k$ between segments of Burgers vector $\boldsymbol{b}$ we denote with $\boldsymbol{l}_{k}^{\boldsymbol{b}+}$ and $\boldsymbol{l}_{k}^{\boldsymbol{b}-}$ the unit tangents before and after the node, respectively

\footnotetext{
${ }^{5}$ This definition excludes junction nodes, which are no interconnects between segments of the same Burgers vector. Because we will focus on a single slip system in the sequel, this is immaterial in the current work. The treatment of junctions is subject of ongoing research.
} 
(remark that the segments are oriented). With this notation the segment- and node-based definitions of the density and curvature tensors read

$$
\begin{aligned}
& \overline{\boldsymbol{\rho}}^{(n) \mathrm{d}, \boldsymbol{b}}(V)=\frac{1}{|V|} \sum_{m=1}^{M(\boldsymbol{b})} L_{m}^{\boldsymbol{b}} \underbrace{\boldsymbol{l}_{m}^{\boldsymbol{b}} \otimes \ldots \otimes \boldsymbol{l}_{m}^{\boldsymbol{b}}}_{n \text {-times }}, \\
& \overline{\boldsymbol{q}}^{(n) \mathrm{d}, \boldsymbol{b}}(V)=\frac{1}{|V|} \frac{1}{n} \sum_{k=1}^{K(\boldsymbol{b})}(\underbrace{\boldsymbol{l}_{k}^{\boldsymbol{b}+} \otimes \ldots \otimes \boldsymbol{l}_{k}^{\boldsymbol{b}+}}_{n-\text { times }}-\underbrace{\boldsymbol{l}_{k}^{\boldsymbol{b}} \otimes \ldots \otimes \boldsymbol{l}_{k}^{\boldsymbol{b}}-}_{n \text {-times }}), \\
& \overline{\boldsymbol{q}}^{*(n) \mathrm{d}, \boldsymbol{b}}(V)=\frac{1}{|V|} \sum_{k=1}^{K(\boldsymbol{b})} \frac{1}{\left|\boldsymbol{l}_{k}^{\boldsymbol{b}+}+\boldsymbol{l}_{k}^{\boldsymbol{b}-}\right|} \operatorname{sym}(\boldsymbol{l}_{k}^{\boldsymbol{b}-} \times \underbrace{\boldsymbol{l}_{k}^{\boldsymbol{b}+} \otimes \ldots \otimes \boldsymbol{l}_{k}^{\boldsymbol{b}+}}_{n \text {-times }}-\boldsymbol{l}_{k}^{\boldsymbol{b}+} \times \underbrace{\boldsymbol{l}_{k}^{\boldsymbol{b}-} \otimes \ldots \otimes \boldsymbol{l}_{k}^{\boldsymbol{b}}}_{n \text {-times }}) .
\end{aligned}
$$

For a pair of subvolumes $V$ and $V^{\prime}$ the pair density tensors are then clearly obtained as

$$
\overline{\boldsymbol{\rho}}^{\left(n, n^{\prime}\right) \mathrm{d}, \boldsymbol{b}, \boldsymbol{b}^{\prime}}\left(V, V^{\prime}\right)=\overline{\boldsymbol{\rho}}^{(n) \mathrm{d}, \boldsymbol{b}}(V) \otimes \overline{\boldsymbol{\rho}}^{\left(n^{\prime}\right) \mathrm{d}, \boldsymbol{b}^{\prime}}\left(V^{\prime}\right),
$$

and according formulas hold for the further pair quantities listed in Eq. (25).

In the current work, dislocation data may either refer to artificially generated random loops or segment based dislocation networks obtained as output from DDD codes, which is ParaDis (Arsenlis et al. 2007) in the present case. After acquiring the dislocation data from either of the two sources we employ an in-house Python code for the correlation analysis, which includes the following major tasks:

1. Reconstruction and visualization of the dislocation configuration

2. Discretization (creation of subvolumes) of the simulation box with prescribed spatial resolution

3. Calculation of spatially resolved alignment and curvature tensors

4. Evaluation of pair density and pair correlation tensors

In case of dislocation networks from the DDD code, nodal data files imported from ParaDis are used as input for the Python code. The employed data file from ParaDis contains all information required for the reconstruction of the dislocation network and subsequent analysis including global domain size, nodal coordinates, neighboring nodes, associated Burgers vector and slip plane normal. For the calculation of spatially resolved alignment tensors (alignment tensor for each subvolume), we applied the definitions discussed above on regular grids of subvolumes. However, we additionally apply a spatial homogenization, such that eventually single-point quantities are constant and correlation quantities only depend on the distance vector between the volumes. In the following, we discuss two different strategies to evaluate pair density tensors which are both used to calculate pair correlation tensors according to Eq. (26).

\section{Numerical schemes to evaluate correlations from line data}

For a numerical computation of the dislocation pair densities in $d=2$ or 3 dimensions, we discretize the computational domain $\Omega$ using a Cartesian grid with $n_{\mathrm{c}}$ cells (also called subvolumes) per coordinate direction, yielding a total number of $N_{\mathrm{c}}=n_{\mathrm{c}}^{d}$ cells. If a dislocation segment spans along multiple subvolumes, it is dissected into two independent 
segments at each boundary between subvolumes, so that each segment can unambiguously be assigned to a cell $V_{i}$ with centerpoint $\boldsymbol{x}_{i}$, where $i \in\left\{1, \ldots, N_{\mathrm{c}}\right\}$. The spatially discretized single-point densities are calculated using Eq. (95) to (97), where the average density of the subvolume is taken as approximation for the density at the centerpoint, i.e. $\boldsymbol{\rho}^{(n) \mathrm{d}, \boldsymbol{b}}\left(\boldsymbol{x}_{i}\right):=\overline{\boldsymbol{\rho}}^{(n) \mathrm{d}, \boldsymbol{b}}\left(V_{i}\right)$.

The discretization and spatial homogenization of Eq. (98) then reads

$$
\boldsymbol{\rho}^{\left(n, n^{\prime}\right) \mathrm{d}, \boldsymbol{b}, \boldsymbol{b}^{\prime}}(\boldsymbol{r})=\frac{1}{|\Omega|} \sum_{j=1}^{N_{\mathrm{c}}} \boldsymbol{\rho}^{(n) \mathrm{d}, \boldsymbol{b}}\left(\boldsymbol{x}_{j}\right) \otimes \boldsymbol{\rho}^{\left(n^{\prime}\right) \mathrm{d}, \boldsymbol{b}^{\prime}}\left(\boldsymbol{x}_{j}+\boldsymbol{r}\right)\left|V_{j}\right|,
$$

where $r$ runs through the discrete set of distance vectors between the center points of the subvolumes, respecting the periodicity of the simulation domain $\Omega$. From a computational point of view, it is simpler to perform a nested loop over all points $\boldsymbol{x}_{i}^{\prime}$ and $\boldsymbol{x}_{j}$ in space and add the contribution to the pair density at the corresponding point in the discrete correlation space $\boldsymbol{r}=\boldsymbol{x}_{i}^{\prime}-\boldsymbol{x}_{j}$ on the fly. This procedure is displayed in Algorithm 1.

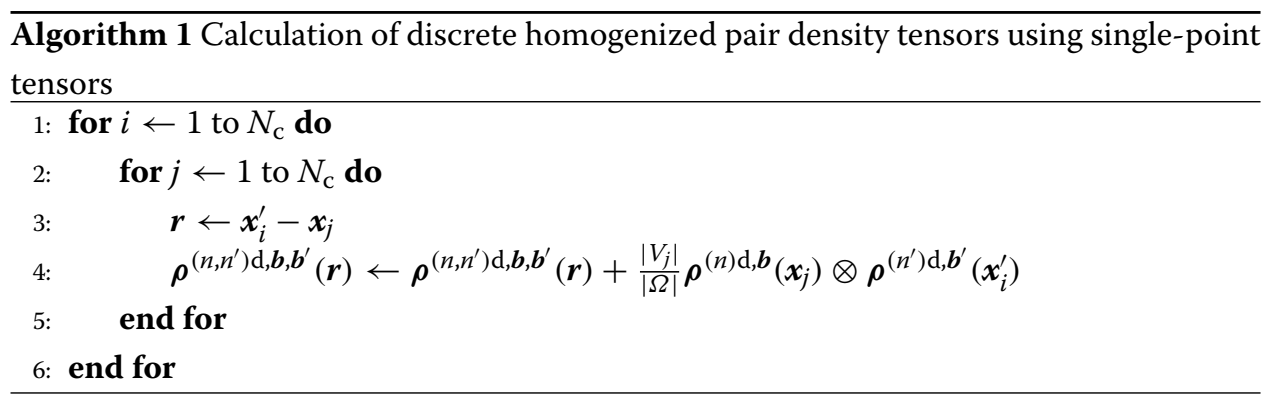

In a typical dislocation configuration with a suitable spatial resolution, a large amount of subvolumes does not contain any dislocation line, which makes Algorithm 1 rather inefficient. We therefore use an equivalent approach directly looping over all pairs of segments, see Algorithm 2. The finer the spatial resolution of the domain, the stronger the gain in performance. With $n_{\mathrm{c}}$ being the number of subvolumes per coordinate direction, the number of subvolumes changes with $\mathcal{O}\left(n_{\mathrm{c}}^{d}\right)$ in $d$ dimensions, while the number of dislocation segments $M$ increases approximately with $\mathcal{O}\left(n_{\mathrm{c}}\right)$ due to the dissecting of segments at subvolume boundaries.

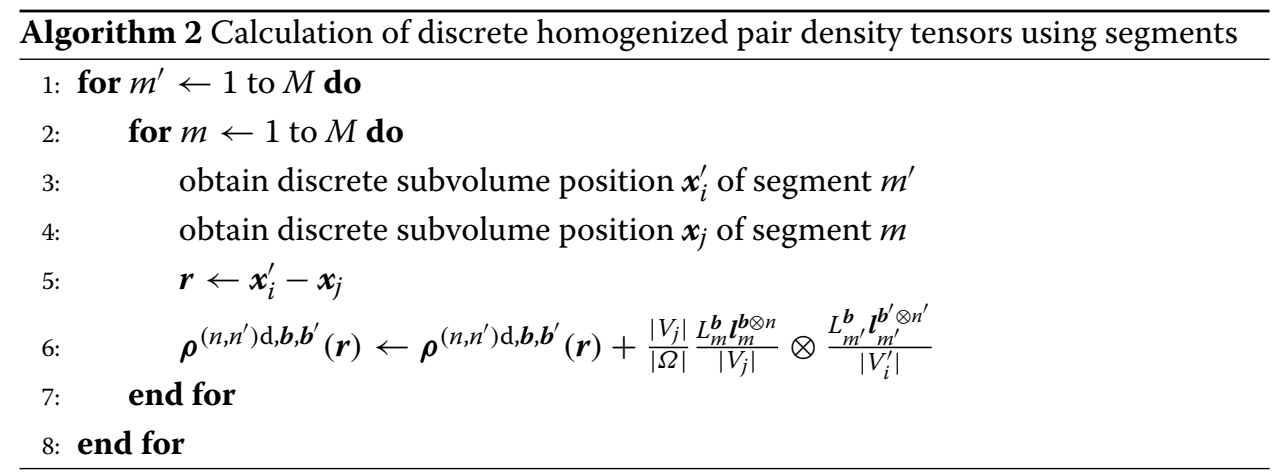


Independent of the employed algorithm, the ensemble averaging over $N$ realizations is straight-forward and directly reflects the theoretical definition,

$$
\boldsymbol{\rho}^{\left(n, n^{\prime}\right) \boldsymbol{b}, \boldsymbol{b}^{\prime}}(\boldsymbol{r})=\left\langle\boldsymbol{\rho}^{\left(n, n^{\prime}\right) \mathrm{d}, \boldsymbol{b}, \boldsymbol{b}^{\prime}}(\boldsymbol{r})\right\rangle=\frac{1}{N} \sum_{i}^{N} \boldsymbol{\rho}_{i}^{\left(n, n^{\prime}\right) \mathrm{d}, \boldsymbol{b}, \boldsymbol{b}^{\prime}}(\boldsymbol{r}) .
$$

The correlation tensors of the ensemble are accordingly obtained using Eqs. 26 and 27 as

$$
\boldsymbol{d}^{\left(n, n^{\prime}\right) \boldsymbol{b}, \boldsymbol{b}^{\prime}}(\boldsymbol{r})=\frac{1}{\rho^{2}}\left(\boldsymbol{\rho}^{\left(n, n^{\prime}\right) \boldsymbol{b}, \boldsymbol{b}^{\prime}}(\boldsymbol{r})-\boldsymbol{\rho}^{(n) \boldsymbol{b}} \otimes \boldsymbol{\rho}^{\left(n^{\prime}\right) \boldsymbol{b}^{\prime}}\right),
$$

where $\boldsymbol{\rho}^{(n) \boldsymbol{b}}$ denote the ensemble and spatially averaged tensors

$$
\boldsymbol{\rho}^{(n) \boldsymbol{b}}=\left\langle\overline{\boldsymbol{\rho}}^{(n) \boldsymbol{b}}(\Omega)\right\rangle=\frac{1}{N} \sum_{i}^{N} \overline{\boldsymbol{\rho}}_{i}^{(n) \mathrm{d}, \boldsymbol{b}}(\Omega)=\frac{1}{N} \frac{1}{N_{\mathrm{c}}} \sum_{i}^{N} \sum_{j}^{N_{\mathrm{c}}} \frac{\left|V_{j}\right|}{|\Omega|} \boldsymbol{\rho}_{i}^{(n) \mathrm{d}, \boldsymbol{b}}\left(\boldsymbol{x}_{j}\right) .
$$

All correlation tensors involving the curvature-related quantities $\boldsymbol{q}^{(n) \boldsymbol{b}}$ and $\boldsymbol{q}^{*(n) \boldsymbol{b}}$ may be evaluated accordingly.

\section{Validation: self-correlations of randomly distributed loops with constant radius}

In order to test the presented numerical scheme, we apply Algorithm 2 to a configuration with known analytical solution. We choose the case of randomly distributed circles with fixed radius $R$ and homogeneously distributed random centers from Section Correlations in 'uncorrelated' distributions of circular loops. Note that one may also look at this the other way round, namely as a verification of the analytical results.

Each loop is approximated as a regular polygon with 50 segments. The length of each segment is calculated using the requirement that the circumference of each loop equals $2 \pi R$. In order to avoid artifacts from this discretization, the angular orientation of the polygon is random for each loop.

We employ a periodic domain, where one has to make sure that the domain size is larger than $4 R$ in each coordinate direction in order to capture all relevant phenomena of this setup. In our example, we set the domain size to $5 R$. The domain is discretized with 301 subvolumes in each coordinate direction. While the number of loops in each realization defines the density of the configuration, the quality of the numerical solution is defined by the number of configurations in the ensemble. An ensemble of $N=1000$ realizations with $N_{\mathrm{l}}=200$ loops each was found to provide good statistics in the current case. This yields the center point density $\rho_{\mathrm{c}}=N_{\mathrm{l}} / V$ and the total dislocation density of $\rho=2 \pi R N_{\mathrm{l}} / V$, and $q=2 \pi N_{\mathrm{l}} / V$. For a quantitative comparison with the analytical solution from Section Correlations in 'uncorrelated' distributions of circular loops, we work with the radius $R$ as length unit, such that $R=1$. This also renders all derived measures, such as dislocations density $\rho$ and pair density $\rho^{(0,0)}$, virtually non-dimensional.

In Fig. 10 the ensemble averaged spatially resolved single-point density $\rho(\boldsymbol{x})$, the pair density $\rho^{(0,0)}(\boldsymbol{r})$, and the correlated GND vector field $\boldsymbol{\rho}^{(0,1)}(\boldsymbol{r})$ are displayed on the right hand side, Fig. 10d to $\mathrm{f}$. To demonstrate the importance of a sufficiently large ensemble, we juxtapose the results to those obtained from a single realization in Fig. 10a to c. The total dislocation density $\rho(\boldsymbol{x})$ in Fig. 10a of a single realization is heterogeneous, and the individual loops are visible. The homogenized pair density $\rho^{(0,0)}(\boldsymbol{r})$ in Fig. 10b and GND pair density $\rho^{(0,1)}(\boldsymbol{r})$ in Fig. 10c of the same realization are rather noisy, but a pattern becomes apparent. For the total dislocation density $\rho(\boldsymbol{x})$ in Fig. 10d of the ensemble with 1000 realizations, a comparably homogeneous distribution emerges and the assumption 


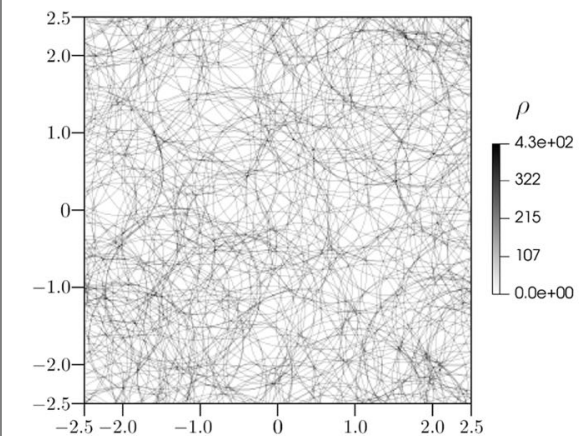

(a)

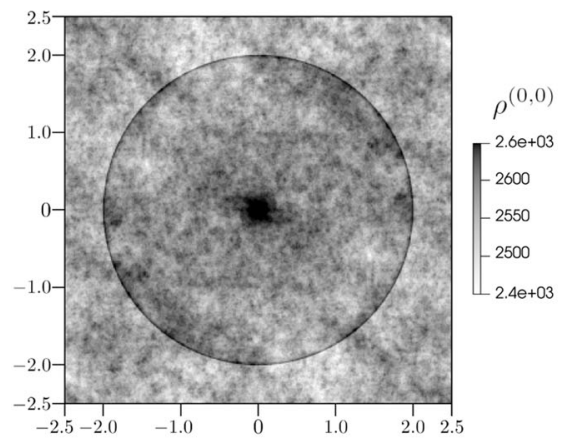

(b)

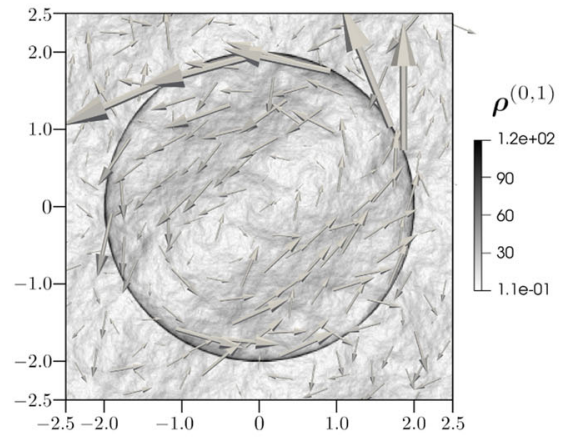

(c)

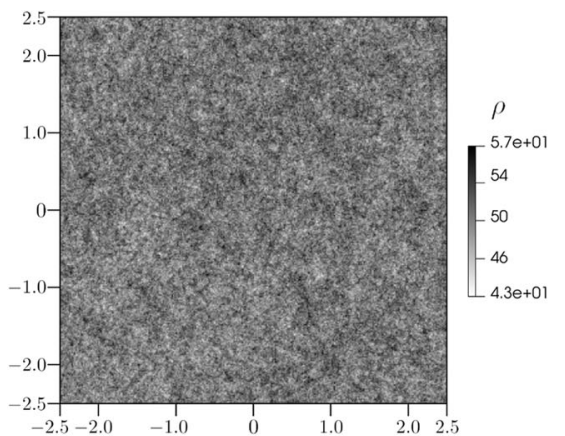

(d)

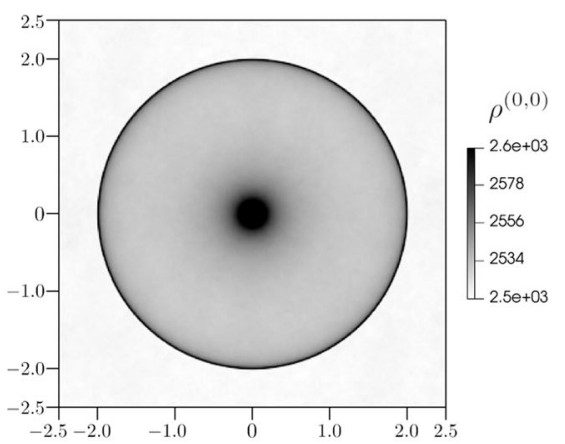

(e)

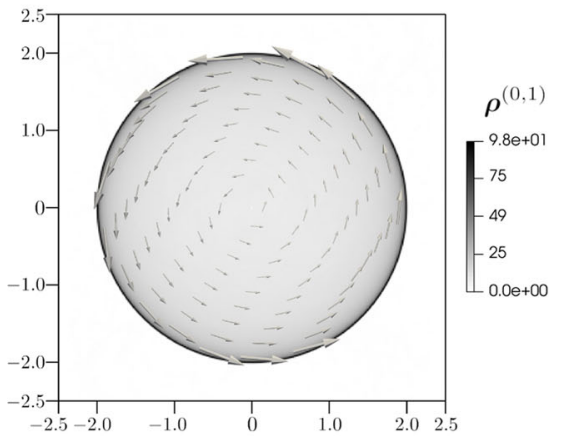

(f)

Fig. 10 Density $\rho$, scalar pair density $\rho^{(0,0)}$ and vector pair density $\boldsymbol{\rho}^{(0,1)}$ of a single realization $(\mathbf{a}$ to $\mathbf{c})$ and of 1000 realizations ( $\mathbf{d}$ to $\mathbf{f}$ ) of a distribution of 200 loops with random center points and radius $R=1$

of an overall constant density $\rho$ seems eligible. To realize this, notice the different scales for one and for 1000 configurations, respectively. The pair density $\rho^{(0,0)}(\boldsymbol{r})$ in Fig. 10e and GND pair density $\boldsymbol{\rho}^{(0,1)}(\boldsymbol{r})$ in Fig. $10 \mathrm{f}$ of the ensemble match the expectation from the analytical solution, compare Fig. 3 for the latter.

For a quantitative comparison with the analytical solution for $\rho^{(0,0)}(\boldsymbol{r})$, which only depends on the distance $r$, the obtained data is averaged over the angular direction via

$$
\rho^{(0,0)}(r)=\frac{1}{2 \pi r} \int_{0}^{2 \pi} \rho^{(0,0)}(r(\boldsymbol{r}), \varphi(\boldsymbol{r})) r \mathrm{~d} \varphi,
$$

with $r(\boldsymbol{r})$ and $\varphi(\boldsymbol{r})$ being the polar coordinates of the position vector $\boldsymbol{r}$ in correlation space. This comparison of analytical and numerical results is shown in Fig. 11, where we 


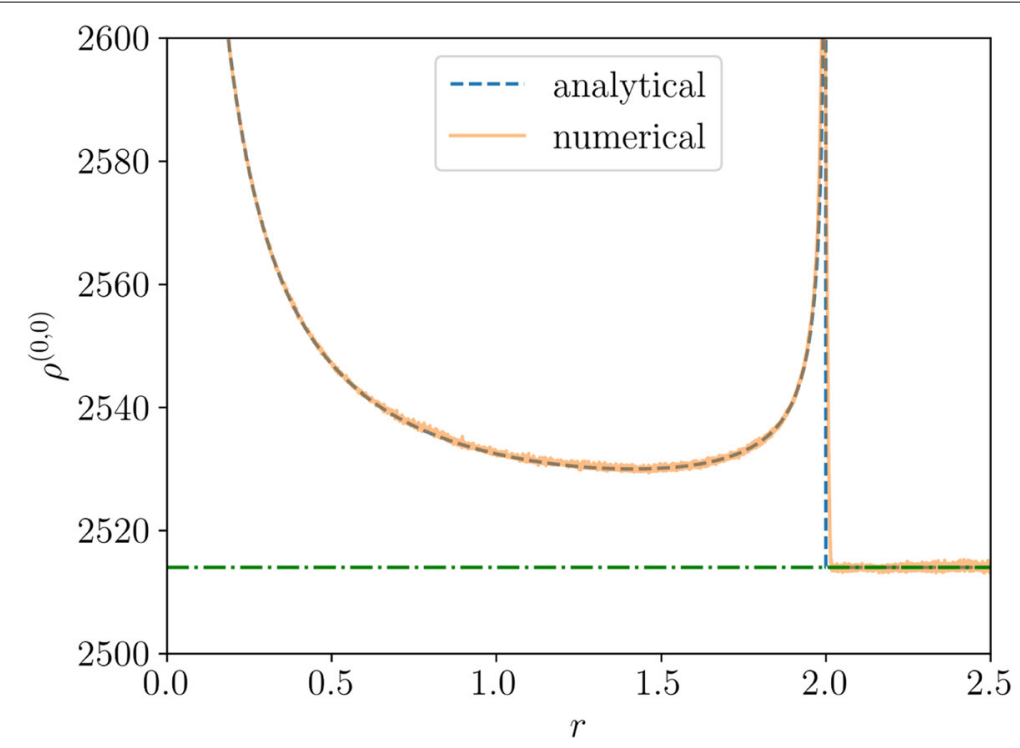

Fig. 11 Numerical and analytical solution for the scalar pair density $\rho^{(0,0)}$ as a function of $r$ in a distributions of loops with radius $R=1$. The dash-dotted green line corresponds to the background pair density $\rho 2 \pi R\left(N_{1}-1\right) / V=2514$

observe an excellent agreement between the two. Note that for the analytical solution we have to slightly modify Eq. (55), since the number of loops in each configuration is finite and the background density contains all loops except one, such that

$$
\rho^{(0,0)}(r)= \begin{cases}\rho \frac{2 R \pi\left(N_{l}-1\right)}{V}+\frac{\rho}{\pi r \sqrt{1-r^{2} /\left(4 R^{2}\right)}} & \text { if } \frac{r}{R} \leq 2, \\ \rho \frac{2 R \pi\left(N_{l}-1\right)}{V} & \text { otherwise. }\end{cases}
$$

The results obtained in this section demonstrate that our algorithm is well-suited for calculating the correlation functions of dislocation networks. The application to a configuration obtained from DDD simulations is topic of the next section.

\section{Ensemble generation for single slip dislocation configurations}

After validating our algorithm against the didactic case of randomly distributed perfect circular loops with constant radius, we apply the same strategy for actual dislocation configurations generated by DDD simulations, in this case using the code ParaDis. For the artificial loop distribution in the previous section, the ensemble was well-defined through the fixed loop geometry and the given number of randomly placed loops. In terms of the CDD variables we thus fixed the whole hierarchy of 'macroscopic' variables $\boldsymbol{\rho}^{(n)}$ and $\boldsymbol{q}^{(n)}$, with the only non-trivial elements $\rho^{(0)}=\rho$ and $q^{(0)}=\rho / R$, all higher order tensors may be reduced to. When evaluating actual DDD simulations, the definition of the right ensemble is less obvious. On the one hand, the right ensemble depends on the envisaged purpose, as is well-known from statistical thermodynamics. On the other hand, when obtaining realizations as results of DDD simulations, one will usually have limited control over the macroscopic variables, like density and curvature tensors. Moreover, dislocation distributions are known to be strongly history dependent, such that the loading history (e.g. strain path) will usually have to be considered as a kind of state variable for the ensemble, even though it may not be a measurable state variable in the usual sense. We 
consider the proper choice of an ensemble for DDD simulations an in general unresolved issue which deserves further research in the future.

For the time being, we resort to a strongly simplified system of dislocations to define an ensemble as follows: (i) we only consider a single slip system, which (ii) is originally populated by a given number of randomly distributed loops of the of same size, then (iii) loaded in uniaxial tension (with fixed loading axis relative to the slip system) in displacement control to a given strain, then (iv) the load is removed and the system reaches a 'sufficiently' relaxed state (see below for definition). As of now we only consider for further analysis those results of the above sketched procedure, where (v) the relaxed state has a total dislocation density (line length) in a given range. The decision to work with relaxed states is motivated by the fact, that for not too high strain rates dislocation systems relax on much smaller time scales than defined by the loading and thus deformation always proceeds through a series of nearly relaxed dislocation states. Similar ensembles have been intensively studied for quasi 2D systems of straight edge dislocations by Zaiser et al. (2001); Groma et al. (2003); Valdenaire et al. (2016). Though the consideration of a single slip system is very restrictive, we note that hexagonal crystals may be deformed by primarily activating one of the basal slip systems, which does in principle allow for checking aspects of the current results experimentally.

In the following we describe the relaxation procedure for a single dislocation configuration, which was employed for the whole ensemble (300 configurations) of statistically similar dislocation configurations. We start with initial configurations containing 50 single slip glide loops of constant radius in a 3D periodic simulation box (see Fig. 12a) generated by the preprocessing utility of ParaDis. All loops are oriented with the same line sense, which ensures that they expand upon the prescribed loading. In the coordinate system defined by the periodic simulation box, the direction of the slip plane normal is $\boldsymbol{e}_{\mathrm{n}} \approx\left[\begin{array}{lll}0.5153 & 0.6285 & 0.5826\end{array}{ }^{T}\right.$ and the slip direction, i.e. the direction of screw dislocations, is $\boldsymbol{e}_{\mathrm{s}}=\boldsymbol{b} /|\boldsymbol{b}| \approx\left[\begin{array}{lll}-0.3052 & 0.7698 & -0.5606\end{array}\right]^{T}$. This corresponds to the (1 111$)\left[\begin{array}{lll}0 & 1 & \overline{1}\end{array}\right]$ slip system where the crystal coordinate system is rotated relative to the laboratory frame to prevent the dislocations from leaving and re-entering the periodic domain on only a handful of distinct planes. The basis vectors of the laboratory frame relative to the crystal coordinate system are provided in Table 1.

The procedure of obtaining a relaxed configuration is done in two steps: in the first step, we load the initial dislocation configuration in tension in $Z$-direction of the laboratory frame ${ }^{6}$ up to a total strain of $\approx 1.15 \%$ (1000 time steps with adaptive time stepping) using the DDD parameters listed in Table 1 for achieving a peak dislocation density in the simulation box, as exemplarily shown in Fig. 12b. In the second step, the externally applied stress is set to zero for relaxation to take place, thanks to the long range interactions between dislocation segments. Note that relaxation is a much slower process as compared to the high strain rate loading. Therefore, we use another, faster time step integrator for relaxation (Sills et al. 2016), as denoted in Table 1. The primary criterion for labeling a dislocation configuration as a relaxed configuration is the complete absence of dislocation activities in the simulation box for a considerable amount of simulation time (for example $30 \%$ of the total simulation time). The fulfillment of this criterion during

${ }^{6}$ Note that in Table 1 we provide the notation of the ParaDis input file, which seems to suggest loading in $\left[\begin{array}{ll}0 & 0\end{array}\right]$-direction of the crystal coordinate system; but the provided coordinates actually refer to the laboratory frame. 


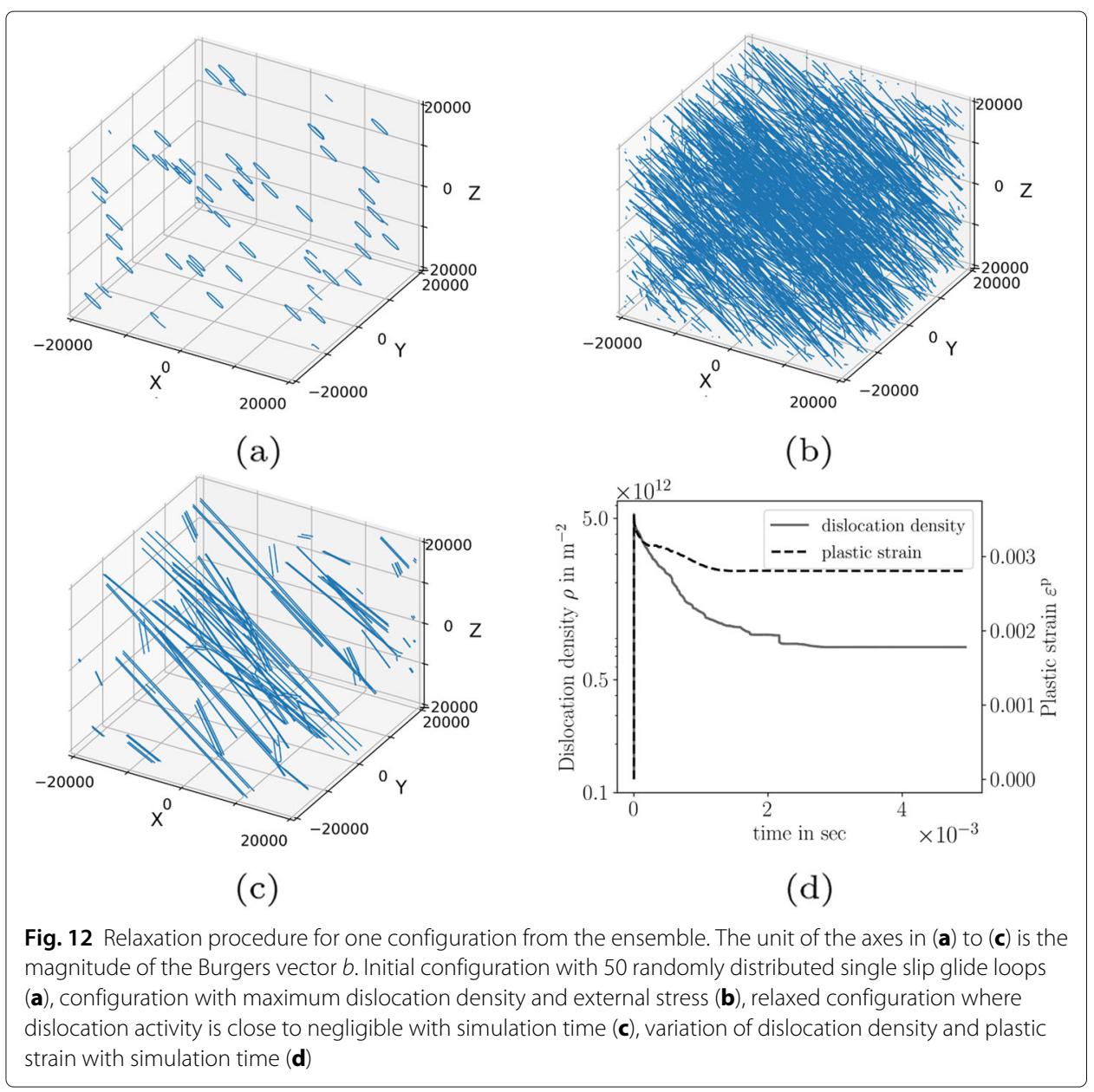

relaxation in the displayed example may be read from Fig. 12d, where the time evolution of total dislocation density and plastic strain are shown. Both values are constant for a long period of simulation time before the simulation was stopped. Note that constant scalar density alone is not a sufficient criterion for the relaxation, because the straight dislocations which develop in the course of relaxation may produce plastic strain without altering their line length. Therefore, we additionally monitor plastic strain as an indicator for dislocation motion.

The above described procedure for obtaining relaxed configurations does not ensure that the relaxed configurations would share the same total dislocation density, or any higher order alignment tensor except for the GND vector $\rho$. This vector vanishes in the initial conditions and remains zero throughout loading and relaxation, i.e. we have $\rho \equiv 0$. Despite the inability to control the total dislocation density we found that a major part of the simulations results in similar total dislocation density between $10^{11}$ to $10^{12} \mathrm{~m}^{-2}$. As a practical definition, we define the ensemble to consist of relaxed configurations with average scalar densities ranging from $4.0 \times 10^{11}$ to $8.0 \times 10^{11} \mathrm{~m}^{-2}$. In the current case this yields an average total density of $\rho \approx 5.76 \times 10^{11} \mathrm{~m}^{-2}$, which we employ as the defining macroscopic density for the ensemble in the sequel. In Fig. 13a the time evolution of the density of the selected simulations is depicted to demonstrate the long time that the 
Table 1 DDD simulation parameters used to obtain a relaxed configuration of dislocations. Hyphens in the column 'Relaxation step' indicate unchanged status with respect to the 'Loading step'

\begin{tabular}{|c|c|c|c|}
\hline Parameters/Test conditions & Loading step & Relaxation step & Unit \\
\hline Simulation domain & $40000 \times 40000 \times 40000$ & - & $|\boldsymbol{b}|$ \\
\hline Mobility law & "FCC_O" & - & - \\
\hline Burgers vector magnitude & $2.55 \times 10^{-10}$ & - & $\mathrm{m}$ \\
\hline Shear modulus & $54.6 \times 10^{9}$ & - & $\mathrm{Pa}$ \\
\hline Poisson's ratio & 0.324 & - & - \\
\hline Fast Multipole Method (FMM) & enabled & - & - \\
\hline FMM cellular Grid & $8 \times 8 \times 8$ & - & - \\
\hline Time step integrator & "trapezoid" & "subcycling" & - \\
\hline Maximum segment length & 4000.0 & - & $|\boldsymbol{b}|$ \\
\hline Edge dislocation mobility & $1.0 \times 10^{3}$ & - & $\mathrm{Pa}^{-1} \mathrm{~s}^{-1}$ \\
\hline Screw dislocation mobility & $1.0 \times 10^{3}$ & - & $\mathrm{Pa}^{-1} \mathrm{~s}^{-1}$ \\
\hline Load type & constant strain rate & creep & - \\
\hline Maximum position error & 1.0 & - & $|\boldsymbol{b}|$ \\
\hline Strain rate & $4.0 \times 10^{4}$ in $\left[\begin{array}{lll}0 & 0 & 1\end{array}\right]$-direction & 0.0 & $s^{-1}$ \\
\hline Applied stress & - & 0.0 & $\mathrm{~Pa}$ \\
\hline Cross-slip & disabled & - & - \\
\hline useLabFrame & enabled & - & - \\
\hline labFrameXDir & {$\left[\begin{array}{lll}0.9514 & -0.2452 & 0.1864\end{array}\right]^{\top}$} & - & - \\
\hline labFrameYDir & {$\left[\begin{array}{lll}0.2722 & 0.9526 & -0.1361\end{array}\right]^{T}$} & - & - \\
\hline labFrameZDir & {$\left[\begin{array}{lll}-0.1441 & 0.1802 & 0.9730\end{array}\right]^{T}$} & - & - \\
\hline
\end{tabular}

density remained constant in all cases. For completeness, we also provide the accumulated plastic strain of the ensemble in Fig. 13b. While this plot illustrates that the dislocation activity ceased for all configurations, it also demonstrates that the plastic strain shows a huge scatter and, therefore, is not a suitable macroscopic quantity of the ensemble.

Besides the need to define the ensemble, we of course also face the question, as to how many realizations are needed in order to have sufficient statistics for evaluating pair correlations. The number may usually not be determined upfront but one has to employ certain statistical measures of the ensemble as indicators. The configurations which we obtain are mostly bundles of straight dipole-like pairs of dislocations which may be of different character. The orientation of the dislocations features prominently in the pair density information. As will be detailed below, we observe a dominance of screw and near screw orientations over more edge type orientations. Since the pair statistics with 300 realizations is still limited, we did not study the convergence of the pair density fields. As a necessary criterion for the representativeness of the pair density data we instead checked the convergence of the average second order dislocation density tensor of the ensemble, which is likewise sensitive to the dipole orientations.

To check the convergence we evaluated the averaged second order alignment tensors. Figure 13 shows the alignment tensors for increasing numbers of relaxed configurations visualized as ellipses in the slip plane with the eigendirections as main axes scaled by the associated eigenvalue. Note that due to single slip dislocation configurations, the third eigenvalue is negligible compared to the other two. Surprisingly, the ellipse obtained from just 10 configurations is already reasonably close to the final one for 300 configurations, which is additionally highlighted by the gray filling, and the ellipses largely appear to approach the final one with increasing number of configurations. This is also supported 


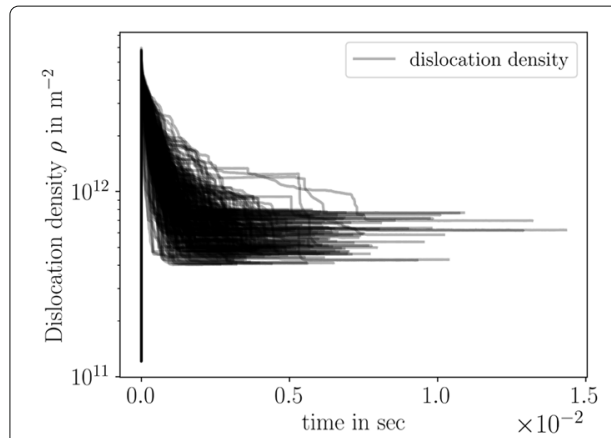

(a)

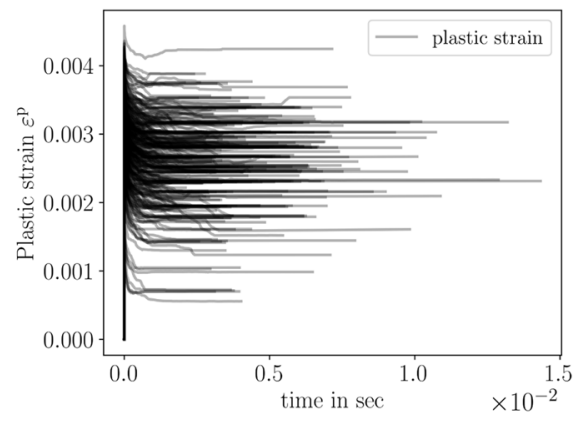

(b)

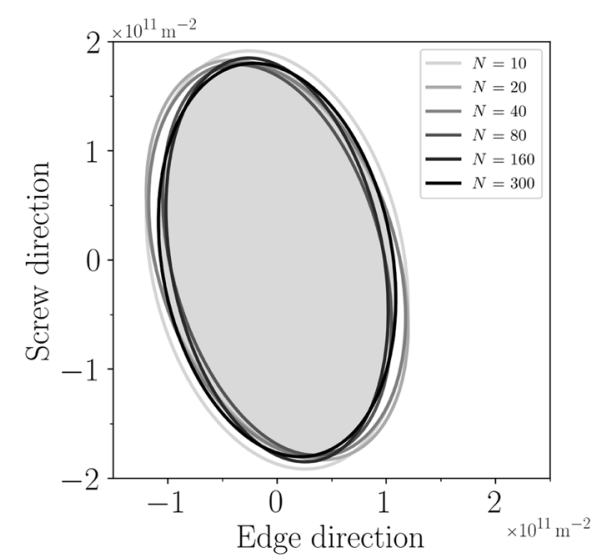

(c)

Fig. 13 The dislocation ensemble considered in the present study consists of statistically similar dislocation configurations which is manifested through: the time evolution of scalar dislocation density for all configurations considered in the ensemble during relaxation (a), the time evolution of plastic strain during the relaxation procedure for the ensemble $(\mathbf{b})$, the convergence of the averaged second order alignment tensors represented as ellipse in the slip plane with increasing number of configurations $N$ considered for averaging (c). Compare Table 2 for the associated eigenvalues and eigenvectors of the averaged second order alignment tensors $\boldsymbol{\rho}^{(2)}$. The ellipse filled in gray represents the second order alignment tensor of the final ensemble $(N=300)$

more quantitatively by the associated eigenvalues and eigenvectors which are listed in Table 2, where the eigenvectors are provided based on unit vectors in screw, $\boldsymbol{e}_{\mathrm{s}}$, edge, $\boldsymbol{e}_{\mathrm{e}}$, and slip plane normal direction, $\boldsymbol{e}_{\mathrm{n}}$. The relative (Frobenius) distance of the two last alignment tensors, i.e., for $N=160$ and 300 configurations, is

$$
\frac{\left\|\boldsymbol{\rho}_{\langle 160\rangle}^{(2)}-\boldsymbol{\rho}_{\langle 300\rangle}^{(2)}\right\|}{\left\|\boldsymbol{\rho}_{\langle 300\rangle}^{(2)}\right\|} \approx 4.7 \%,
$$

where the norm symbol denotes the Frobenius norm for matrices. We consider this change upon roughly doubling the number of realizations as small enough to regard the total density and directional information as sufficiently converged. We therefore expect that also the correlation data is more than a random snapshot and displays certain features of the ensemble. 
Table 2 Eigenvalues and eigenvectors of averaged second order alignment tensors obtained using ensembles with increasing number of configurations (presented in the slip system coordinate)

\begin{tabular}{|c|c|}
\hline Eigenvalue & Eigenvector \\
\hline \multicolumn{2}{|c|}{ Ensemble with 10 configurations } \\
\hline$\lambda_{1}=3.8835 \times 10^{11}$ & $\boldsymbol{e}_{1}=0.9783 \boldsymbol{e}_{\mathrm{s}}-0.2071 \boldsymbol{e}_{\mathrm{e}}$ \\
\hline$\lambda_{2}=2.3082 \times 10^{11}$ & $\boldsymbol{e}_{2}=0.2071 \boldsymbol{e}_{\mathrm{s}}+0.9783 \boldsymbol{e}_{\mathrm{e}}$ \\
\hline$\lambda_{3}=8.7028 \times 10^{5}$ & $\boldsymbol{e}_{3}=\boldsymbol{e}_{\mathrm{n}}$ \\
\hline \multicolumn{2}{|c|}{ Ensemble with 20 configurations } \\
\hline$\lambda_{1}=3.7984 \times 10^{11}$ & $\boldsymbol{e}_{1}=0.9460 \boldsymbol{e}_{\mathrm{s}}-0.3241 \boldsymbol{e}_{\mathrm{e}}$ \\
\hline$\lambda_{2}=2.1753 \times 10^{11}$ & $\boldsymbol{e}_{2}=0.3241 \boldsymbol{e}_{\mathrm{s}}+0.9460 \boldsymbol{e}_{\mathrm{e}}$ \\
\hline$\lambda_{3}=9.8537 \times 10^{5}$ & $\boldsymbol{e}_{3}=\boldsymbol{e}_{\mathrm{n}}$ \\
\hline \multicolumn{2}{|c|}{ Ensemble with 40 configurations } \\
\hline$\lambda_{1}=3.7104 \times 10^{11}$ & $\boldsymbol{e}_{1}=0.9569 \boldsymbol{e}_{5}-0.2902 \boldsymbol{e}_{\mathrm{e}}$ \\
\hline$\lambda_{2}=2.1857 \times 10^{11}$ & $\boldsymbol{e}_{2}=0.2902 \boldsymbol{e}_{\mathrm{s}}+0.9569 \boldsymbol{e}_{\mathrm{e}}$ \\
\hline$\lambda_{3}=1.1124 \times 10^{6}$ & $\boldsymbol{e}_{3}=\boldsymbol{e}_{\mathrm{n}}$ \\
\hline \multicolumn{2}{|c|}{ Ensemble with 80 configurations } \\
\hline$\lambda_{1}=3.6672 \times 10^{11}$ & $\boldsymbol{e}_{1}=0.9650 \boldsymbol{e}_{5}-0.2621 \boldsymbol{e}_{\mathrm{e}}$ \\
\hline$\lambda_{2}=1.9299 \times 10^{11}$ & $\boldsymbol{e}_{2}=0.2621 \boldsymbol{e}_{\mathrm{s}}+0.9650 \boldsymbol{e}_{\mathrm{e}}$ \\
\hline$\lambda_{3}=1.0255 \times 10^{6}$ & $\boldsymbol{e}_{3}=\boldsymbol{e}_{\mathrm{n}}$ \\
\hline \multicolumn{2}{|c|}{ Ensemble with 160 configurations } \\
\hline$\lambda_{1}=3.7478 \times 10^{11}$ & $\boldsymbol{e}_{1}=0.9819 \boldsymbol{e}_{\mathrm{s}}-0.1895 \boldsymbol{e}_{\mathrm{e}}$ \\
\hline$\lambda_{2}=1.9428 \times 10^{11}$ & $\boldsymbol{e}_{2}=0.1895 \boldsymbol{e}_{5}+0.9819 \boldsymbol{e}_{\mathrm{e}}$ \\
\hline$\lambda_{3}=1.0130 \times 10^{6}$ & $\boldsymbol{e}_{3}=\boldsymbol{e}_{\mathrm{n}}$ \\
\hline \multicolumn{2}{|c|}{ Ensemble with 300 configurations } \\
\hline$\lambda_{1}=3.6427 \times 10^{11}$ & $\boldsymbol{e}_{1}=0.9841 \boldsymbol{e}_{5}-0.1774 \boldsymbol{e}_{\mathrm{e}}$ \\
\hline$\lambda_{2}=2.1089 \times 10^{11}$ & $\boldsymbol{e}_{2}=0.1774 \boldsymbol{e}_{5}+0.9841 \boldsymbol{e}_{\mathrm{e}}$ \\
\hline$\lambda_{3}=1.0613 \times 10^{6}$ & $\boldsymbol{e}_{3}=\boldsymbol{e}_{\mathrm{n}}$ \\
\hline
\end{tabular}

Before analyzing the pair density and correlation data we note that already the average alignment tensor contains some non-trivial information about the ensemble. That the average alignment tensor is not a spherical tensor in the slip plane but possesses two clearly distinct eigenvalues shows that there are preferred orientations in which the dislocations form dipoles. In this case the eigenvector to the lager eigenvalue is nearly in screw while the other (accordingly) nearly in edge direction, which indicates a preference for screw dipoles. That (near) screw dipoles exist is presumably because we did not allow for cross slip in the current investigation. Cross slip would facilitate the annihilation of screw dipoles such that a dominance of edge dipoles would rather be expected. The dominance of the screw direction in the case without cross slip may be rationalized from the fact that -in case of elastic isotropy as employed in the ParaDis code- for the same slip plane spacing the passing stress of two straight screw dislocations is a factor of 1.4 higher than for two straight edge dislocations. Screw segments accordingly are expected to get more easily tied in dipole configurations. Moreover, the energy per line length is minimal for screw dislocations (Hirth and Lothe 1968). However, the main axis with the largest eigenvalue is in the current simulations consistently found to be about $10^{\circ}$ off the screw direction. While this is unsatisfactory and needs to be further investigated before drawing any physical conclusions, this result seems of lesser importance for the current, rather didactical paper. This issue is briefly reconsidered in Section Summary, discussion, and outlook section. 


\section{Correlation analysis of relaxed single slip configurations}

In all configurations of our ensemble $(N=300)$, we observe that most of the dislocations self-arrange in straight dipoles, similar to the example shown in Fig. 12c. Since cross-slip is deactivated, no junctions occur. The creation of dipoles with two straight, parallel dislocation lines on distinct planes which are very close to each other is the only mechanism which prevents the original loops (which stay non-circular closed loops after loading) from shrinking and disappearing due to line tension during relaxation. Changes of line direction, i.e. curvature, appear only in small regions when dislocations are involved in several straight dipoles and turn from one dipole direction to the other.

One goal of the current paper is to show that the correlation data is dominated by connectivity information and to provide perspectives on how to separate this from other interaction information between dislocations. Note though, that the tendency of dislocations to be straight, is likewise the results of (self-)interactions between segments. We begin the analysis with looking at the self-interactions, which in the current single slip case means, we regard the correlation information in the plane spanned by distance vectors in the slip plane in correlation space - that is, a plane parallel to the slip plane through the origin.

In Fig. 14a we plot the scalar correlation function $d^{(0,0)}$ in this slice as obtained via Algorithm 2 with 121 subvolumes in each coordinate direction. This plot shows a peak at the origin, while the fading density at larger distances fans out into linear maxima. The peak in the center is elongated in the screw direction and also the linear maxima appear to lie preferentially around the screw, rather than the edge direction. Note that the correlation data provides additional information to the orientation information already contained in the second order alignment tensor $\boldsymbol{\rho}^{(2)}$, mostly because it displays the straightness of the dislocations.

That the correlation in the regarded slip plane slice is essentially the connectivity information discussed in Section Correlations in 'uncorrelated' distributions of circular loops shall be checked by comparing the angular average of the pair density in the plane with the analytical result for isotropically distributed straight lines. This comparison is provided

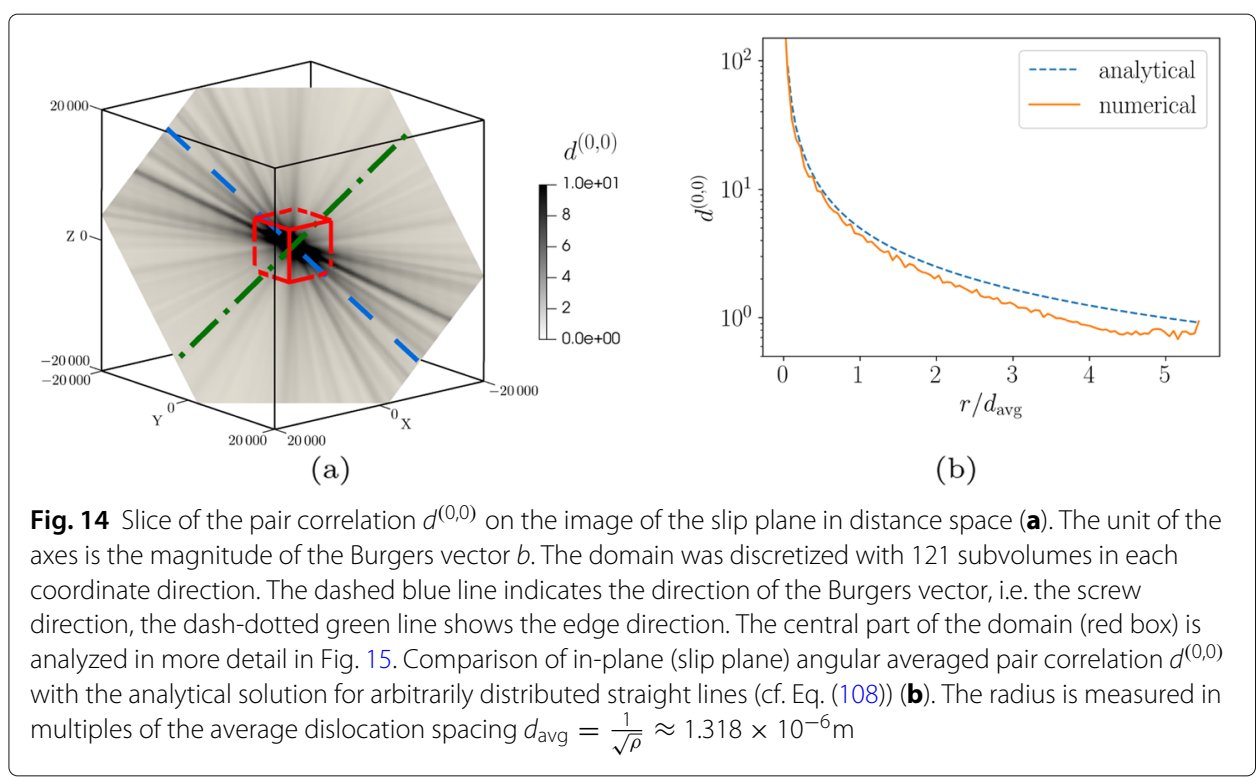


in Fig. 14b. Since we deal with a three-dimensional example, we have to slightly modify the method for obtaining the analytical solution as compared to Section Correlations in 'uncorrelated' distributions of circular loops, and take into account a certain extension $h$ in the direction of the slip plane normal. We now consider the line length $L(r)$ contained inside a cylinder with radius $r$ and height $h$, which is given for the case of uncorrelated straight dislocation lines by

$$
L(r)=\rho \pi r^{2} h+2 r .
$$

In analogy to Eq. (55), we calculate the pair density as

$$
\rho^{(0,0)}(r)=\frac{\rho}{2 \pi r h} \frac{\mathrm{d} L(r)}{\mathrm{d} r}=\rho^{2}+\frac{\rho}{\pi r h},
$$

which results in the pair correlation function

$$
d^{(0,0)}(r)=\frac{1}{\rho^{2}}\left(\rho^{(0,0)}-\rho^{2}\right)=\frac{1}{\rho \pi r h} .
$$

In the current analysis, the height $h$ is chosen as the side length of one subvolume, i.e. $h=\left(|\Omega| / N_{\mathrm{C}}\right)^{\frac{1}{3}}$, where $|\Omega|$ is the volume of the computational domain and $N_{\mathrm{C}}$ is the total number of subvolumes. From now on, all quantities with unit of length will be given as multiples of the average dislocation spacing, which is $d_{\text {avg }}=\frac{1}{\sqrt{\rho}} \approx 1.318 \times 10^{-6} \mathrm{~m}$, since this is the intrinsic length of our ensemble. Fig. 14b shows that the analytical and the numerical simulation fit excellently in the central region of the correlation space, but they slightly diverge after a few average dislocation spacing. One reason might be that not all dislocations are straight lines. On the other hand, since the slip plane has been oriented in the periodic unit cell such that its periodic copies do not overlap, dislocations seem to leave the slip plane at the boundary of the unit cell. This numerical artifact might be involved in the clear under-estimation of pair density at distances approaching the order of the box size of $1.02 \times 10^{-5} \mathrm{~m} \approx 7.735 \mathrm{~d}_{\mathrm{avg}}$. In the sequel we shall therefore concentrate on the correlations only in a small box around the origin.

For the subsequent analysis we resolved the central part of the correlation domain (red cube in Fig. 14 with side length $2.042 \times 10^{-6} \mathrm{~m} \approx 1.548 d_{\text {avg }}$, of about one fifth of the unit cell) with 1001 subvolumes in each coordinate direction. The density plot on the slice along the slip plane in Fig. 15a shows a similar shape as Fig. 14a, however with a finer resolution. Since we are closer to the center, the values of the correlation $d^{(0,0)}$ are higher and the color scale is different from the one in Fig. 14a. To provide an impression of the results outside the slip plane, we plot an isosurface with a constant scalar correlation $d^{(0,0)}$ of 75 in Fig. 15b. While this plot is not by itself very helpful, it clearly indicates that also the $3 \mathrm{D}$ data seems to be strongly dominated by the self-correlations concentrated in the image of the slip-plane through the center. In Fig. 15c and d we show, respectively, a zoomed in and a rotated view of the same isosurface. Preferred directions of dipoles are clearly visible in the linear protuberances parallel to the slip plane. The screw direction is roughly aligned with the long linear hump along the diagonal in Fig. 15c. As may be read from Fig. 15c, no protuberances occur in directions with an obtuse angle to the screw direction. Note, however, that we indeed also observe dipoles of edge-like dislocations in the relaxed configurations, but the low density of edge-like orientations already 'in the slip plane' prevents them from being reflected in the current plot.

Because of the inherent difficulty in visualizing and analyzing 3D field data, we provide several 2D projections of the correlation function in Fig. 16. For this purpose we 


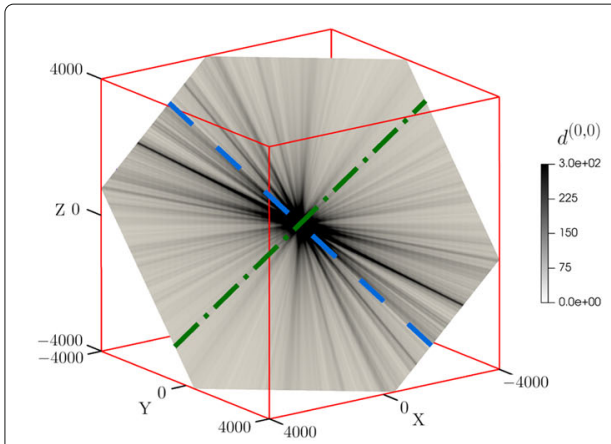

(a)

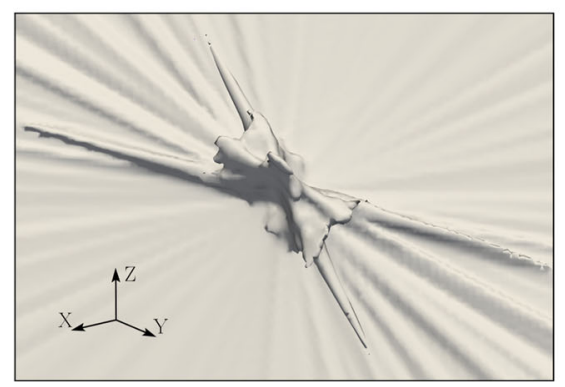

(c)

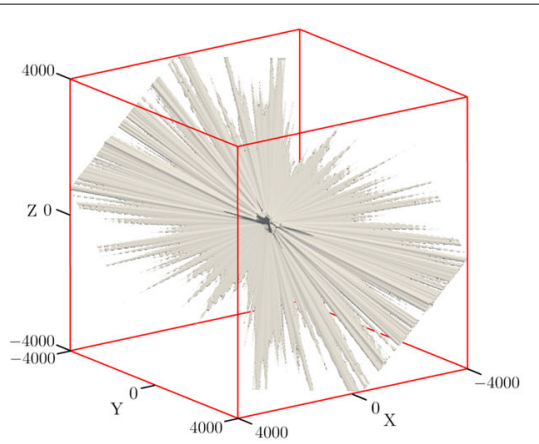

(b)

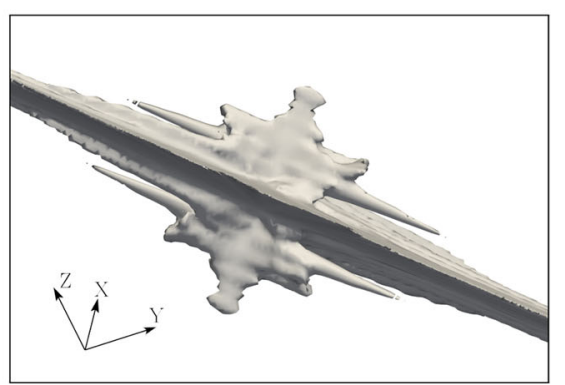

(d)

Fig. 15 Correlation $d^{(0,0)}$ in the central part of the correlation space (cf. Fig. 14a). The unit of the axes in (a) and $(\mathbf{b})$ is the magnitude of the Burgers vector $b$. The slice of the pair correlation $d^{(0,0)}$ on the image of the slip plane in distance space is shown in (a). The dashed blue line indicates the direction of the Burgers vector, i.e. the screw direction. The dash-dotted green line shows the edge direction. Surface with constant correlation of 75 (b). Zoom of the center of the contour plot (c). Rotated view of the central part of the contour plot, parallel to the slip plane (d)

transform the three-dimensional data from the Cartesian grid to a cylindrical coordinate system with coordinates $(r, \varphi, z)$. The origin of this new coordinate system is the center of the correlation domain, the reference direction for the angle coincides with the screw direction, and the reference plane is the slip plane, such that $z$ gives the normal distance to the central plane parallel to the slip plane. Figure 16a displays the angular average of the correlation function as a function of in-plane distance $r$ and normal distance $z$. Considering the point symmetry of the function, only positive $z$ directions are displayed. This data shows very high values in the slip plane and for small $z$-values, which is due to the in-plane self-correlations and the narrow dipoles. Note that due to the periodicity of the calculation domain, the slip planes re-enter the computational domain at a position shifted in slip plane normal direction. The repeated re-entry happens at different $z$-values. These re-entering copies may in the case of straight dislocations contain pieces of the same dislocation, such that in the correlation analysis these 'self-correlations' contribute to correlations at $z>0$. The $z$-positions of several low order re-entry planes are indicated by dash-dotted lines in Fig. 16a. The numbers accompanying these lines indicate what number copy appears at the $z$-value. In the named plot one may suspect that around the plane through the origin there is a narrow strip which is devoid of pair correlations. When analyzing this with higher resolution in $z$-direction we found that this empty range approximately corresponds to the annihilation distance of $d_{\text {ann }}=20|\boldsymbol{b}|=5.1 \times 10^{-9} \mathrm{~m}$ employed in ParaDis, see Fig. 16b. Due to this gap, one may for the current ensemble 


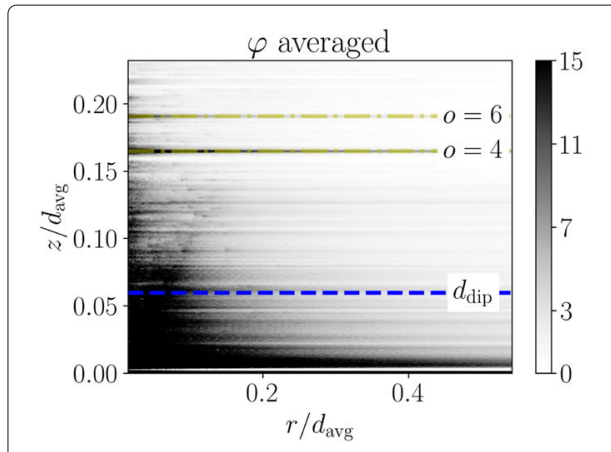

(a)

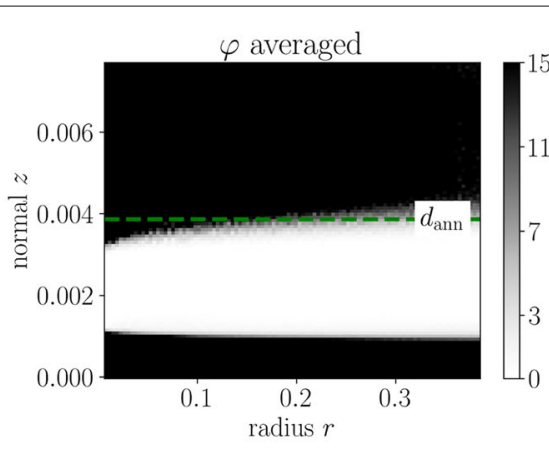

(b)

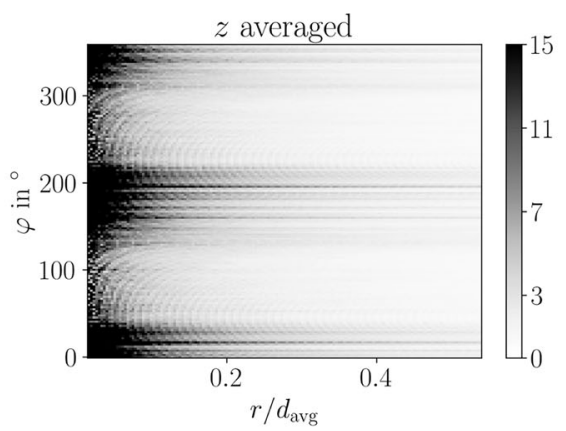

(c)

Fig. 16 Surface plots of the correlation function $d^{(0,0)}$ in a cylindrical coordinate system aligned with the slip plane. The values for $r$ and $z$ are given in multiples of the average dislocation spacing $d_{\text {avg }}=\frac{1}{\sqrt{\rho}}$. Averaged over angular domain (a). The dashed line is the average dipole height (cf. Eq. (109)), the dash-dotted lines indicate planes re-entering the domain due to periodicity of order 0 , and therefore contain self-correlation. Gap in the correlation in normal direction (zoomed in z-direction of (a) using a finer spatial resolution) (b). The out-of-plane pair correlation starts approximately at the annihilation distance $d_{\text {ann }}$ of the DDD code. Correlation averaged over normal direction $z(\mathbf{c})$

completely separate the self-correlations in the slip plane from pair correlations between segments on neighboring planes.

We employ this separation to calculate a simple approximation of the average dipole height as

$$
d_{\text {dip }}=\frac{\int_{z_{0}}^{\infty} z d^{(0,0)}(r=0, z) \mathrm{d} z}{\int_{z_{0}}^{\infty} d^{(0,0)}(r=0, z) \mathrm{d} z}
$$

where we choose the lower limit $z_{0} \approx 0.0025 d_{\text {avg }}$ of the integral to lie within the above described gap in the correlation. As upper limit we used the maximum $z$ value of the cylindrical coordinate system $z_{\max } \approx 0.7518 d_{\mathrm{avg}}$. As also indicated in Fig. 16a, the average dipole height of our ensemble is approximately $d_{\text {dip }} \approx 7.875 \times 10^{-8} \mathrm{~m} \approx 0.05972 d_{\text {avg }}$. That is, the dipole height is much smaller than the average dislocation spacing by a factor of about 17 .

Figure 16c shows the correlation averaged over the $z$-direction within the cylindrical volume. The restriction to $z$-values markedly smaller than the edge length of the periodic unit cell avoids finite size effects. The data averaged in $z$-direction is a function of radius $r$ and angle $\varphi$ measured from the Burgers vector direction. The data is $\pi$ periodic, but it is displayed for the full range of angles. This plot again confirms the dominance of near 
screw dislocations in the ensemble, and displays the straight character in the long 'tails' seen especially for the high density orientations.

For a quantitative analysis of the data we regard the scaling of the correlations with distance $r$ as a function of distance $z$, and the scaling with height $z$ as functions of distance $r$. As discussed with reference to Fig. 14b, a power law scaling is observed within the slip plane near the center. In Fig. 17a, the angle-averaged correlation is given as a function of $r$ for multiple values of $z$. Each of the curves is fitted against a power law of type

$$
d^{(0,0) \mathrm{fit}}(r, z)=a(z) r^{m(z)},
$$

similar to the form of Eq. (108), which applies for $z=0$ with exponent $m(0)=-1$. For the fitting we only start at $r=0.01 d_{\text {avg }}$ in order to exclude effects of the singularity at $r=0$.

With increasing values of $z$ the power law fitting becomes less appropriate. At a normal distance of $z>0.499 d_{\text {avg }}$ the density appears to be essentially uncorrelated. In Fig. 17b the exponent $m(z)$ and the coefficient $a(z)$ of the power law fitting are given as a function of normal distance $z$. For the self-correlations $(z=0)$ the exponent is -1 as expected. It approaches zero with increasing distance from the center-plane. Large wiggling of the obtained values starts at $z \approx 0.3 d_{\text {avg }}$, which indicates a breakdown of the power law scaling. To substantiate this claim we use the relative fitting error,

$$
e=\frac{\sqrt{\sum_{i}\left(x_{i}^{\mathrm{fit}}-x_{i}^{\mathrm{data}}\right)^{2}}}{\sqrt{\sum_{i}\left(x_{i}^{\mathrm{data}}\right)^{2}}} .
$$

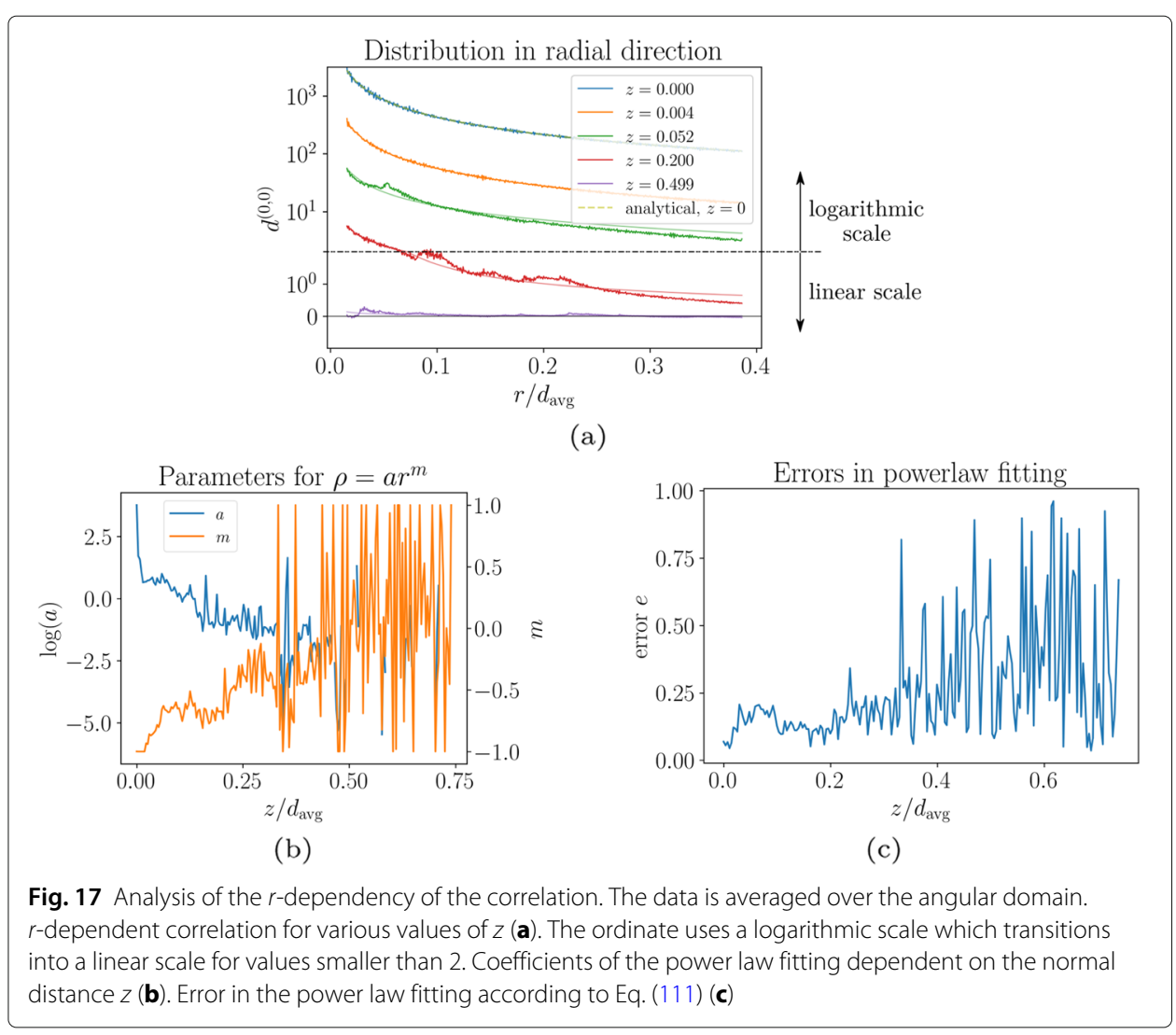


This error is depicted for the obtained power law fits as a function of $z$ in Fig. 17c. Also the relative error markedly increases and starts oscillating with high amplitude for $z>$ $0.3 d_{\text {avg }}$, thus confirming the breakdown of the power law. While the quality of fitting the radial dependence of the correlation with the simple power law (Eq. (110)) may be questionable for increasing values of $z$, our analysis shows that correlations show a power law scaling near the center within the slip plane and in its vicinity. This reflects the long narrow dipoles observed in the relaxed configurations.

As opposed to the relatively long ranged correlations parallel to the slip plane we observe clearly short ranged correlations with exponential decay in slip plane normal direction. The early breakdown of the power law fitting discussed in the preceding paragraph may be viewed as a first indication of this fast decay in $z$-direction. Figure 18a shows the angle-averaged correlation as a function of $z$ for multiple values of $r$. Each of the curves is fitted against an exponential law of type

$$
d^{(0,0) \mathrm{fit}}(z, r)=a(r) \exp (b(r) z),
$$

where we start the fitting at $z=0.025 d_{\text {avg }}$ in order to exclude effects of the self-correlation at $z=0$. The fitting parameters of the exponential law are given in Fig. 17b as a function of radial distance $r$. While the coefficient $a$ steadily decreases, the exponential coefficient $b$ starts at $r \approx 0$ with large negative value of -20 , and quickly drops to -10 at $r \approx 0.1 d_{\text {avg }}$, where it remains for increasing values of $r$. The comparatively smooth $r$-dependency of

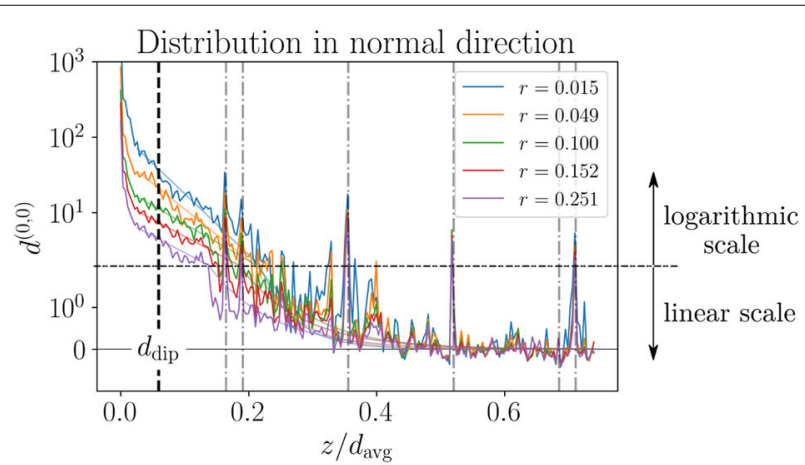

(a)

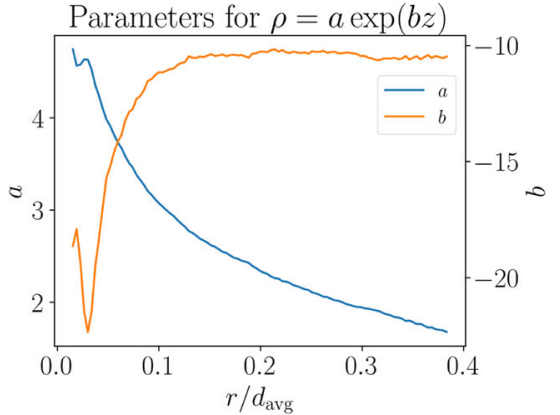

(b)

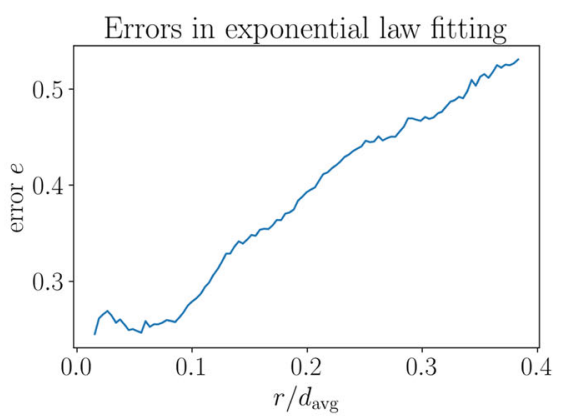

(c)

Fig. 18 Analysis of the $z$-dependency of the correlations based on the data averaged over the angular domain. $z$-dependent correlation for various values of $r(\mathbf{a})$. The black dashed line indicates the average dipole height, cf. Eq. (109). The gray dash-dotted lines indicate peaks due to re-entrance of in-plane self-correlation through the periodic domain. The ordinate uses a logarithmic scale which transitions into a linear scale for values smaller than 2. Coefficients of the exponential fitting dependent on the radial in-plane distance $r(\mathbf{b})$. Error $e$ in the exponential fitting according to Eq. (111) (c) 
the coefficients indicates that the exponential fit is well suited for analyzing the decay of the correlations in $z$-direction. However, for increasing values of $r$, the error $e$ becomes larger, as shown in Fig. 18c. Though this relative fitting error appears large, our analysis clearly reveals the short range character of the pair correlation in $z$-direction.

\section{Summary, discussion, and outlook}

In the current paper, we introduced the characterization of dislocation pair correlations based on a series of two-point tensors in the space of distance vectors, which are built upon the alignment tensor concept employed for characterizing single-point dislocation density information. Special emphasis was given on the consequences of spatial homogenization of pair density information already on the discrete level. Homogenization leads to a markedly different representation of pair density information on the space of distance vectors than on the product space of point pairs. This difference arises from the extended nature of dislocations and is closely connected to the self-correlations which occur in systems of closed curves. These self-correlations were derived analytically for a two-dimensional model system of uncorrelated circles; highlighting at the same time the content of several low order correlation tensors. These analytical results were then confirmed by analyzing numerically generated distributions of uncorrelated circles, serving at the same time as validation for the numerical algorithms for evaluating correlations based on straight segments. Subsequently, these algorithms were employed for analyzing correlations in an ensemble of relaxed single slip dislocation systems obtained by DDD simulations. In this case, self-correlations and pair correlations from elastic interactions could be mostly distinguished and analyzed separately. In the relaxed configurations we observed $1 / r$-type self-correlations near the origin which could be well explained by the analytical result for randomly oriented straight lines. Correlations of power law type close to the origin extended also to small distances perpendicular to the slip plane, as a result of the formation of long straight narrow dipoles during relaxation. Correlations were found to be short ranged perpendicular to the slip plane.

The central motivation for the current paper is to clarify the peculiarities of correlations in dislocation systems. We think that it is important to be aware of the dominance of selfcorrelations in dislocation correlation data, and believe that it is beneficial to distinguish these mostly geometric correlations from those resulting from elastic interactions. We note, though, that also the self-correlations are not entirely independent of elastic correlations. For instance, the average dislocation curvature was found to be contained in the correlated GND vector field, while the local curvature of the dislocations is also a consequence of local stresses and elastic interactions. Also the average second order alignment tensor turned out to contain some kind of correlation information in that it reflects preferred orientations which likewise occur in the scalar density-density correlations. To which extend and for which purposes it will be necessary or beneficial to distinguish self and pair correlations remains an open question in our eyes.

While the clear exposition of dislocation correlations is the central theme of this contribution, we shall briefly discuss the analyzed DDD data and the correlations observed therein. We decided to use a single slip system in order to keep the analysis manageable, while there is a perspective to study such systems experimentally in hexagonal crystals oriented for basal slip. However, stabilizing a distribution of dislocations on a single slip system is of course a challenge. The employed slip system orientation and mechanical 
loading successfully generated non-trivial, stable single slip configurations, but in hindsight we caution to assign much physical significance to the presented results. The only conclusions we hypothesize to be transferable are the power law type correlations in and close to the slip plane in 'horizontal' direction and the fast decay of correlations in slip system normal direction. The average dipole height or the preferred orientation, by contrast, may be rather sensitive to the chosen slip system orientation relative to the periodic unit cell: the dipole height is likely to be influenced by the re-entry of periodic copies of the slip planes, and the observed $\approx 10^{\circ}$ deviation of the main axes of the alignment tensor from the only distinguished directions in the slip system, i.e. from the edge and screw directions, obviously lacks a physical justification. The latter is thus presumably related to a break of symmetry in the slip plane when cut by the periodic unit cell. These insights obviously require further investigation and modifications of the ensemble generation when aiming at solid physical conclusions. While the principles of analyzing DDD correlation data introduced in the current paper are not affected by this cautionary note, these considerations restrained us from presenting and analyzing any higher order correlation tensors from the DDD data.

We conclude this work by giving an outlook as to how we believe the correlation data may be of use to promote our understanding of dislocation plasticity and continuum modeling of dislocations. The most obvious application of correlation information relates to the development of a statistical continuum theory of dislocations, similar as the one developed for straight parallel edge dislocations, see Valdenaire et al. (2016); Wu et al. (2018). In this case, pair correlations may be either used to incorporate average interaction effects directly in the force averaging (Zaiser et al. 2001; Groma et al. 2003), or, aiming at an energy based thermodynamic description (Groma et al. 2007; Hochrainer 2016), for modeling the average energy based on a local density approximation (Zaiser 2015). In the quasi 2D theory of parallel edge dislocation, the short-range nature of the pair correlations played a crucial rôle. While the current preliminary investigation seems to confirm the short ranged nature of correlations perpendicular to the slip plane, correlations were longer ranged within and close to the slip plane. How these self-interactions would be reflected in the continuum theory is not yet clear. Note though, that the reason for the apparent long range correlations is the 'preference' of the dislocations to be straight and thus related to line tension. On the energetic level, line tension effects naturally enter the thermodynamic theory of planar curved dislocations due to the coupling of line length changes to the scalar curvature variable $q$, cf. Hochrainer (2016). This connection fosters the interpretation of the curvature variables as carriers of correlation information. While we cautioned to give too much weight to the emergence of preferred orientations we observed in the simulations, we note that similar preferred orientations are known to emerge in real dislocation systems. The incorporation of such correlation effects in a continuum theory has not yet been attempted, but by considering an anisotropic line energy, as suggested by Zaiser (2015), a theory which incorporates the second order alignment tensor as internal variable should see driving forces for rotating the main axes of the tensor to an energetically favorable alignment. If a higher passing stress would be the main reason for selecting preferred orientations, we expect such behavior could be integrated in a continuum theory by an anisotropic friction stress, i.e., a tensorial generalizations of the well-known Taylor stress. 
Eventually, a statistical continuum theory and accordingly the correlation analysis does of course need to address multiple slip configurations with dislocation reactions, junctions, cross slip and possibly jogs due to dislocation cutting. As for the determination of alignment tensors and the according correlation tensors, the presented theory and the algorithms for analyzing DDD data are readily applicable and it will mostly be the high degree of complexity which will be a challenge for data analysis and their incorporation into continuum theories. However, there are still fundamental issues to be resolved with regard to the proper representation of a complex dislocation network in continuum variables. Important steps have been recently taken by considering (different types of) junction node densities as complementary continuum variable to dislocation densities for characterizing dislocation networks (Starkey et al. 2022) in the so-called line density approximation (Lin and El-Azab 2020). The open issues in generalizing this to fully averaged continuum descriptions are closely related to the question of how to transfer the discrete curvature definitions at nodes with discontinuities in the line direction to junction nodes, where more than two dislocations meet.

Aside from the incorporation of dislocation correlations in continuum dislocation theories, the correlation information, if evaluated for (large enough) single realizations, may help characterizing and thus differentiating between different DDD outcomes (or initial conditions), which coincide in their average single-point tensors up to a given order. This may be of crucial importance in interpreting results from DDD simulations, which are known to be dependent on the initial conditions. Relating the difference in outcome to the one in the initial conditions requires quantifying the differences in either configuration. We hypothesize that spatial correlations could yield relevant metrics for this quantification. Such quantitative measures would ideally also be related to macroscopic mechanical properties. Finding the deciding correlation measures and connecting them to macroscopic properties seem to be tasks, which are well-suited for employing modern machine learning algorithms.

\section{Appendix A}

In this Appendix we briefly discuss how the spatial homogenization of a product of $\delta$ distributions on the product space yields a $\delta$-distribution on the difference space. For this we regard distributions at two distinct points from which the case for the curves as employed in Eq. (37) does then follow immediately. This issue requires consideration, because the product of two $\delta$-distributions on the same space is not defined. When integrating the coordinate $\boldsymbol{x}$, which is just shifted by $\boldsymbol{r}$ in one of the arguments, such a product definition apparently occurs.

Let $\boldsymbol{p}$ and $\boldsymbol{q}$ be two points in the periodic unit cell $\Omega \subset \mathbb{R}^{3}$ which may as a limiting case also be the whole $\mathbb{R}^{3}$. Let furthermore $\delta_{\boldsymbol{p}}(\boldsymbol{x})=\delta(\boldsymbol{x}-\boldsymbol{p})$ and $\delta_{\boldsymbol{q}}(\boldsymbol{x})=\delta(\boldsymbol{x}-\boldsymbol{q})$ be the $\delta$-distributions at the two points. Note that these distributions define linear functionals acting on functions $f(\boldsymbol{x})$ by

$$
\delta_{\boldsymbol{p}}[f]=\int_{\Omega} \delta_{\boldsymbol{p}}(\boldsymbol{x}) f(\boldsymbol{x}) d \boldsymbol{x}=f(\boldsymbol{p}) .
$$

The product of the distributions is a well-defined double-distribution on the product space,

$$
\delta_{p} \delta_{q}\left(\boldsymbol{x}, \boldsymbol{x}^{\prime}\right)=\delta_{p}(\boldsymbol{x}) \delta_{q}\left(\boldsymbol{x}^{\prime}\right) .
$$


This double-distribution acts as a functional on functions $f\left(\boldsymbol{x}, \boldsymbol{x}^{\prime}\right)$ on the product space as

$$
\delta_{\boldsymbol{p}} \delta_{q}[f]=\int_{\Omega} \int_{\Omega} \delta_{\boldsymbol{p}}(\boldsymbol{x}) \delta_{\boldsymbol{q}}\left(\boldsymbol{x}^{\prime}\right) f\left(\boldsymbol{x}, \boldsymbol{x}^{\prime}\right) d \boldsymbol{x}^{\prime} d \boldsymbol{x}=\int_{\Omega} \delta_{\boldsymbol{p}}(\boldsymbol{x}) f(\boldsymbol{x}, \boldsymbol{q}) d \boldsymbol{x}=f(\boldsymbol{p}, \boldsymbol{q}) .
$$

The proposition employed in Eq. (37) is that

$$
\int_{\Omega} \delta_{p} \delta_{q}(\boldsymbol{x}, \boldsymbol{x}+\boldsymbol{r}) d \boldsymbol{x}=\delta_{q-p}(\boldsymbol{r}),
$$

as a distribution on distance space. Note again, that the left hand side above is not naturally defined because both distributions share 'the same' -though shifted-argument. To show the proposition we regard the action of the integrated distribution on a function on distance space, $f(\boldsymbol{r})$. This action involves another integral over $\boldsymbol{r}$ in distance space. For these two integrals we assume that Fubini's theorem holds, i.e., that we may switch the order of integration. With the order of integration switched, the integral over distance space may be regularly evaluated, such that also the outer integral becomes well-defined. We thus find

$$
\begin{aligned}
\left(\int_{\Omega} \delta_{\boldsymbol{p}} \delta_{\boldsymbol{q}}(\boldsymbol{x}, \boldsymbol{x}+\boldsymbol{r}) d \boldsymbol{x}\right)[f] & =\int_{\Omega} \int_{\Omega} \delta_{\boldsymbol{p}}(\boldsymbol{x}) \delta_{\boldsymbol{q}}(\boldsymbol{x}+\boldsymbol{r}) f(\boldsymbol{r}) d \boldsymbol{x} d \boldsymbol{r} \\
& =\int_{\Omega} \int_{\Omega} \delta_{\boldsymbol{p}}(\boldsymbol{x}) \delta_{\boldsymbol{q}-\boldsymbol{x}}(\boldsymbol{r}) f(\boldsymbol{r}) d \boldsymbol{r} d \boldsymbol{x} \\
& =\int_{\Omega} \delta_{\boldsymbol{p}}(\boldsymbol{x}) f(\boldsymbol{q}-\boldsymbol{x}) d \boldsymbol{x} \\
& =f(\boldsymbol{q}-\boldsymbol{p})=\delta_{\boldsymbol{q}-\boldsymbol{p}}[f] .
\end{aligned}
$$

Because this equality holds irrespective of the function $f(\boldsymbol{r})$, we conclude that the distributional equality, Eq. (116), holds.

\section{Appendix B}

In this appendix we sketch how parallel straight segments of curves may be described by a singular density in distance space. If two curves $c_{m}^{b}$ and $c_{m^{\prime}}^{b^{\prime}}$ share a common tangent on part of their parametrization domains, $\left[s_{1}, s_{2}\right) \in I$ and $\left[t_{1}, t_{2}\right) \in I^{\prime}$ the surface they span in product space is mapped to a straight line in distance space. The discrete measure in distance space is accordingly concentrated on this line. While this map is singular, we may still define a weighted measure concentrated on the line in distance space. To derive this we reparametrize the parallel curve segments with parameters $\bar{s}, \bar{t} \in[0,1)$, such that

$$
\begin{aligned}
\boldsymbol{c}_{m}^{\boldsymbol{b}}(\bar{s}) & =\boldsymbol{c}_{m}^{\boldsymbol{b}}\left(s_{1}\right)+\left(\boldsymbol{c}_{m}^{\boldsymbol{b}}\left(s_{2}\right)-\boldsymbol{c}_{m}^{\boldsymbol{b}}\left(s_{1}\right)\right) \bar{s} \\
\boldsymbol{c}_{m^{\prime}}^{\boldsymbol{b}^{\prime}}(\bar{t}) & =\boldsymbol{c}_{m^{\prime}}^{\boldsymbol{b}^{\prime}}\left(t_{1}\right)+\left(\boldsymbol{c}_{m^{\prime}}^{\boldsymbol{b}^{\prime}}\left(t_{2}\right)-\boldsymbol{c}_{m^{\prime}}^{\boldsymbol{b}^{\prime}}\left(t_{1}\right)\right) \bar{t}
\end{aligned}
$$

The unit direction is introduced as

$$
\boldsymbol{l}:=\frac{\boldsymbol{c}_{m}^{\boldsymbol{b}}\left(s_{2}\right)-\boldsymbol{c}_{m}^{\boldsymbol{b}}\left(s_{1}\right)}{\left|\boldsymbol{c}_{m}^{\boldsymbol{b}}\left(s_{2}\right)-\boldsymbol{c}_{m}^{\boldsymbol{b}}\left(s_{1}\right)\right|},
$$

and we note that the unit tangent to the other curve is either $\boldsymbol{l}$ or $-\boldsymbol{l}$. For the following consideration the orientation is immaterial and we assume without loss of generality that the tangents are the same, 


$$
\boldsymbol{l}=\frac{\boldsymbol{c}_{m^{\prime}}^{\boldsymbol{b}^{\prime}}\left(t_{2}\right)-\boldsymbol{c}_{m^{\prime}}^{\boldsymbol{b}^{\prime}}\left(t_{1}\right)}{\left|\boldsymbol{c}_{m^{\prime}}^{\boldsymbol{b}^{\prime}}\left(t_{2}\right)-\boldsymbol{c}_{m^{\prime}}^{\boldsymbol{b}^{\prime}}\left(t_{1}\right)\right|} .
$$

We furthermore state that the length of the segments are given by

$$
L_{m}=\left|\boldsymbol{c}_{m}^{\boldsymbol{b}}\left(s_{2}\right)-\boldsymbol{c}_{m}^{\boldsymbol{b}}\left(s_{1}\right)\right| \quad \text { and } \quad L_{m^{\prime}}=\left|\boldsymbol{c}_{m^{\prime}}^{\boldsymbol{b}^{\prime}}\left(t_{2}\right)-\boldsymbol{c}_{m^{\prime}}^{\boldsymbol{b}^{\prime}}\left(t_{1}\right)\right| .
$$

The ensuing straight line segment $C$ in distance space is covered by the mapping

$$
\begin{aligned}
\boldsymbol{C}(\bar{s}, \bar{t}) & =\boldsymbol{c}_{m^{\prime}}^{\boldsymbol{b}^{\prime}}(\bar{t})-\boldsymbol{c}_{m}^{\boldsymbol{b}}(\bar{s}) \\
& =\boldsymbol{c}_{m^{\prime}}^{\boldsymbol{b}^{\prime}}\left(t_{1}\right)-\boldsymbol{c}_{m}^{\boldsymbol{b}}\left(s_{1}\right)+\left(\bar{t} L_{m^{\prime}}-\bar{s} L_{m}\right) \boldsymbol{l} .
\end{aligned}
$$

The prefactor in front of the line direction $\boldsymbol{l}$ takes values in the interval of $\left[-L_{m}, L_{m^{\prime}}\right)$. We now parametrize the image of the map $C(\bar{s}, \bar{t})$ with a single parameter, $\lambda \in\left[0, L_{m}+L_{m^{\prime}}\right)$, in that

$$
\boldsymbol{C}(\lambda)=\boldsymbol{c}_{m^{\prime}}^{\boldsymbol{b}^{\prime}}\left(t_{1}\right)-\boldsymbol{c}_{m}^{\boldsymbol{b}}\left(s_{1}\right)+\left(\lambda-L_{m}\right) \boldsymbol{l} .
$$

The density with which we need to weight the points on this curve, which corresponds to the function $\phi(s, t)$ in the surface case, now is determined by how many pairs of points on the two curves map to a given $\lambda$ value. By equating Eqs. 127 and 128 and assuming $\lambda$ and $\bar{s}$ given, we may solve for $\bar{t}$ as

$$
\bar{t}(\lambda, \bar{s})=\frac{\lambda+(\bar{s}-1) L_{m}}{L_{m^{\prime}}} .
$$

The total covering of the point $C(\lambda)$ is given by the range of $\bar{s}$ values for which $\bar{t}(\lambda, \bar{s}) \in$ $[0,1)$. The density function $\phi(\lambda)$ is then defined as

$$
\phi(\lambda)=L_{m} \int_{0}^{1} 1_{[0,1)}(\bar{t}(\lambda, \bar{s})) \mathrm{d} \bar{s},
$$

where $1_{[0,1)}(x)$ denotes the characteristic function of the unit interval, which is 1 if $x \in$ $[0,1)$ and zero else. Without loss of generality we assume that $L_{m^{\prime}} \geq L_{m}$. The integral equals the content of the range of values $\bar{s} \in[0,1)$ for which $\bar{t}(\lambda, \bar{s}) \in[0,1)$. Inserting the right hand side of Eq. (129) into $0 \leq \bar{t}<1$ yields the inequalities

$$
\frac{L_{m}-\lambda}{L_{m}} \leq \bar{s} \leq \frac{L_{m}+L_{m^{\prime}}-\lambda}{L_{m}},
$$

from which the range of suitable $\bar{s}$-values may be obtained. Together with the requirement that $\bar{s}$ be between 0 and 1 these inequalities yield three distinct regions for $\lambda$ : if $\lambda \leq L_{m}$ the left limit is larger than zero, while the right limit is larger than one and therefore does not impose an extra restriction. The range of $\bar{s}$-values in this case depends on $\lambda$ and is given by $\lambda / L_{m}$. If $L_{m}<\lambda \leq L_{m^{\prime}}$, the left limit is smaller than zero and the right limit larger than one, such that the range of $\bar{s}$ is 1 . If $L_{m^{\prime}}<\lambda \leq L_{m}+L_{m^{\prime}}$ the left limit is smaller than zero, but the right limit is smaller than one. The according $\bar{s}$-range is again a function of $\lambda$, i.e. $\left(L_{m}+L_{m^{\prime}}-\lambda\right) / L_{m}$. As a consequence, the density function is found as

$$
\phi(\lambda)= \begin{cases}\lambda, & \text { for } 0 \leq \lambda \leq L_{m}, \\ L_{m}, & \text { for } L_{m}<\lambda \leq L_{m^{\prime}}, \\ L_{m}+L_{m^{\prime}}-\lambda, & \text { for } L_{m^{\prime}}<\lambda \leq L_{m}+L_{m^{\prime}} .\end{cases}
$$

The apparent asymmetry in this condition regarding the role of $L_{m}$ and $L_{m^{\prime}}$ stems from assuming $L_{m} \leq L_{m^{\prime}}$. A more general version of Eq. (132) may be obtained through exchanging $L_{m}$ by $\min \left(L_{m}, L_{m^{\prime}}\right)$ and $L_{m^{\prime}}$ by $\max \left(L_{m}, L_{m^{\prime}}\right)$. 
For two parallel curve segments we consequently obtain the discrete pair density in distance space as

$$
\boldsymbol{\rho}^{\mathrm{d}\left(n, n^{\prime}\right) \boldsymbol{b}, \boldsymbol{b}^{\prime}}(\boldsymbol{r})=\frac{1}{|\Omega|} \int_{0}^{L_{m}+L_{m^{\prime}}} \phi(\lambda) \delta(\boldsymbol{r}-\boldsymbol{C}(\lambda)) \boldsymbol{l}_{m}^{\boldsymbol{b} \otimes n}(s) \otimes \boldsymbol{l}_{m^{\prime}}^{\boldsymbol{b}^{\prime} \otimes n^{\prime}}(t) \mathrm{d} \lambda .
$$

As a simple confirmation of this modeling we check that for a volume $R$ in distance space which contains the complete line of distance vectors we have

$$
\int_{R} \rho^{\mathrm{d}(0,0) \boldsymbol{b}, \boldsymbol{b}^{\prime}}(\boldsymbol{r}) \mathrm{d} \boldsymbol{r}=\frac{1}{|\Omega|} \int_{0}^{L_{m}+L_{m^{\prime}}} \phi(\lambda) \mathrm{d} \lambda=\frac{L_{m} L_{m^{\prime}}}{|\Omega|},
$$

which is, upon multiplication with $|\Omega|$, in accordance with the integral of the two-point density over a volume in product space which contains both curve segments.

\section{Abbreviations}

DDD: Discrete Dislocation Dynamics; CDD: Continuum Dislocation Dynamics; GND: Geometrically Necessary Dislocations

\section{Acknowledgments}

We wish to thank Dr. Ryan Sills for providing the latest edition of the ParaDis DDD code with advanced time integration scheme. This research was supported in part through the high performance computing resources provided by the IT services of Graz University of Technology.

\section{Authors' contributions}

TH conceptualized the study and is the major contributor in writing the manuscript. BW performed the data analysis and also contributed to the interpretation of results and writing the manuscript. SG conducted the discrete dislocation dynamics simulations and performed data analysis. All authors read and approved the final manuscript.

\section{Funding}

This research received no specific grant from any funding agency in the public, commercial, or not-for-profit sectors.

\section{Availability of data and materials}

The datasets generated and/or analysed during the current study are available in the Making sense of dislocation correlations - ParaDis data files repository at TU Graz, [DOI:10.3217/an3jq-cdm10]

\section{Declarations}

\section{Competing interests}

The authors declare that they have no competing interests.

Received: 27 October 2021 Accepted: 15 December 2021

Published online: 22 February 2022

\section{References}

J. P. Anderson, A. El-Azab, On the three-dimensional spatial correlations of curved dislocation systems. Mater. Theory. 5(1), 1 (2021). https://doi.org/10.1186/s41313-020-00026-w

A. Arsenlis, W. Cai, M. Tang, M. Rhee, T. Oppelstrup, G. Hommes, T. G. Pierce, V. V. Bulatov, Enabling strain hardening simulations with dislocation dynamics. Model. Simul. Mater. Sci. Eng. 15(6), 553-595 (2007). https://doi.org/10.1088/ 0965-0393/15/6/001

F. F. Csikor, I. Groma, T. Hochrainer, D. Weygand, M. Zaiser, in Proceedings of the 11th International Symposium on Continuum Models and Discrete Systems. ed. by D. Jeulin, S. Forest, On the range of $3 \mathrm{~d}$ dislocation pair correlations (Mines ParisTech Les Presses, Paris, 2007), pp. 271-276

J. Deng, A. El-Azab, Dislocation pair correlations from dislocation dynamics simulations. J. Computer-Aided Mater. Des. 14(1), 295-307 (2007). https://doi.org/10.1007/s10820-008-9090-4

J. Deng, A. El-Azab, Mathematical and computational modelling of correlations in dislocation dynamics. Model. Simul. Mater. Sci. Eng. 17(1), 075010 (2007). https://doi.org/10.1088/0965-0393/17/7/075010

I. Groma, F. F. Csikor, M. Zaiser, Spatial correlations and higher-order gradient terms in a continuum description of dislocation dynamics. Acta Mater. 51, 1271-1281 (2003). https://doi.org/10.1016/S1359-6454(02)00517-7

I. Groma, G. Györgyi, B. Kocsis, Dynamics of course grained disocation densities from an effective free energy. Philos. Mag. 87(8-9), 1185-1199 (2007). https://doi.org/10.1080/14786430600835813

I. Groma, P. D. Ispánovity, T. Hochrainer, Dynamics of curved dislocation ensembles. Phys. Rev. B. 103, 174101 (2021). https://doi.org/10.1103/PhysRevB.103.174101

I. Groma, M. Zaiser, P. D. Ispánovity, Dislocation patterning in a two-dimensional continuum theory of dislocations. Phys. Rev. B. 93, 214110 (2016). https://doi.org/10.1103/PhysRevB.93.214110

J. P. Hirth, J. Lothe, Theory of Dislocations. (McGraw-Hill, New York, 1968)

T. Hochrainer, Multipole expansion of continuum dislocations dynamics in terms of alignment tensors. Philos. Mag. 95(12), 1321-1367 (2015). https://doi.org/10.1080/14786435.2015.1026297 
T. Hochrainer, Thermodynamically consistent continuum dislocation dynamics. J. Mech. Phys. Solids. 88, 12-22 (2016). https://doi.org/10.1016/j.jmps.2015.12.015

T. Hochrainer, On the derivation of boundary conditions for continuum dislocation dynamics. Crystals. 7(8), 235 (2017). https://doi.org/10.3390/cryst7080235

T. Hochrainer, S. Sandfeld, M. Zaiser, P. Gumbsch, Continuum dislocation dynamics: towards a physically theory of plasticity. J. Mech. Phys. Solids. 63, 167-178 (2014). https://doi.org/10.1016/j.jmps.2013.09.012

E. Kröner, Kontinuumstheorie der Versetzungen und Eigenspannungen. (Springer, Berlin, 1958)

E. Kröner, Inelastic behavior of solids. (McGraw-Hill Book Company, New York, 1969), pp. 137-147

E. Kröner, Benefits and shortcomings of the continuous theory of dislocations. Int. J. Solids Structs. 38(6-7), 1115-1134 (2001). https://doi.org/10.1016/S0020-7683(00)00077-9

P. Lin, A. El-Azab, Implementation of annihilation and junction reactions in vector density-based continuum dislocation dynamics. Model. Simul. Mater. Sci. Eng. 28(4), 045003 (2020). https://doi.org/10.1088/1361-651x/ab7d90

M. Monavari, S. Sandfeld, M. Zaiser, Continuum representation of systems of dislocation lines: A general method for deriving closed-form evolution equations. J. Mech. Phys. Solids. 95, 575-601 (2016). https://doi.org/10.1016/j.jmps. 2016.05.009

M. Monavari, M. Zaiser, S. Sandfeld, Comparison of closure approximations for continuous dislocation dynamics. MRS Proc. 1651 (2014). https://doi.org/10.1557/opl.2014.62

R. B. Sills, A. Aghaei, W. Cai, Advanced time integration algorithms for dislocation dynamics simulations of work hardening. Model. Simul. Mater. Sci. Eng. 24(4), 045019 (2016). https://doi.org/10.1088/0965-0393/24/4/045019

K. Starkey, T. Hochrainer, A. El-Azab, Development of mean-field continuum dislocation kinematics with junction reactions using de Rham currents and graph theory. J Mech Phys Solids. 158, 104685 (2022). https://doi.org/10.1016/ j.jmps.2021.104685

H. Stoyan, D. Stoyan, Simple stochastic models for the analysis of dislocation distributions. Phys. Status Solidi (a). 97(1), 163-172 (1986). https://doi.org/10.1002/pssa.2210970114

M. Sudmanns, M. Stricker, D. Weygand, T. Hochrainer, K. Schulz, Dislocation multiplication by cross-slip and glissile reaction in a dislocation based continuum formulation of crystal plasticity. J. Mech. Phys. Solids. 132, 103695 (2019). https://doi.org/10.1016/j.jmps.2019.103695

P.-L. Valdenaire, Y. Le Bouar, B. Appolaire, A. Finel, Density-based crystal plasticity: From the discrete to the continuum. Phys. Rev. B. 93, 214111 (2016). https://doi.org/10.1103/PhysRevB.93.214111

B. Weger, S. Gupta, T. Hochrainer, Analysing discrete dislocation data using alignment and curvature tensors. Compt. Rendus. Phys. (2021). https://doi.org/10.5802/crphys.60

R. Wu, D. Tüzes, P. Ispánovity, I. Groma, T. Hochrainer, M. Zaiser, Instability of dislocation fluxes in a single slip: Deterministic and stochastic models of dislocation patterning. Phys. Rev. B. 98(5), 054110 (2018). https://doi.org/10. 1103/PhysRevB.98.054110

S. Xia, J. Belak, A. El-Azab, The discrete-continuum connection in dislocation dynamics: I. time coarse graining of cross slip. Model. Simul. Mater. Sci. Eng. 24(7), 075007 (2016). https://doi.org/10.1088/0965-0393/24/7/075007

M. Zaiser, Local density approximation for the energy functional of three-dimensional dislocation systems. Phys. Rev. B. 92, 174120 (2015). https://doi.org/10.1103/PhysRevB.92.174120

M. Zaiser, M. C. Miguel, I. Groma, Statistical dynamics of dislocation systems: The influence of dislocation-dislocation correlations. Phys. Rev. B. 64, 224102 (2001). https://doi.org/10.1103/PhysRevB.64.224102

\section{Publisher's Note}

Springer Nature remains neutral with regard to jurisdictional claims in published maps and institutional affiliations.

\section{Submit your manuscript to a SpringerOpen ${ }^{\circ}$ journal and benefit from:}

- Convenient online submission

- Rigorous peer review

- Open access: articles freely available online

- High visibility within the field

- Retaining the copyright to your article

Submit your next manuscript at $\boldsymbol{\triangleright}$ springeropen.com 